\title{
MATERIALS COMPATIBILITY AND LUBRICANTS RESEARCH ON CFC-REFRIGERANT SUBSTITUTES
}

\author{
Quarterly MCLR Program \\ Technical Progress Report
}

1 January 1995 - 31 March 1995

Steven R. Szymurski

Glenn C. Hourahan

David S. Godwin

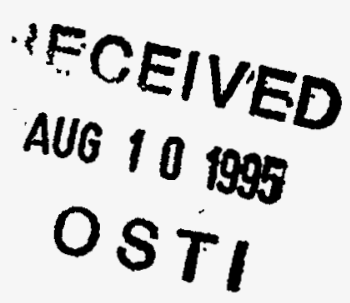

\begin{abstract}
Air-Conditioning and Refrigeration
Technology Institute, Inc.

4301 North Fairfax Drive, Suite 425

Arlington, Virginia 22203
\end{abstract}

April 1995

Prepared for

\section{The U.S. DEPARTMENT OF ENERGY \\ Office of Building Technology \\ Grant Number DE-FG02-91CE23810}

This program is supported, in part, by U.S. Department of Energy grant number DE-FG02-91CE23810: Materials Compatibility and Lubricants Research (MCLR) on CFC-Refrigerant Substitutes. Federal funding supporting this program constitutes $\$ 9,233,000$ or $93.57 \%$ of allowable costs. Funding from non-government sources supporting this program consists of direct cost sharing totaling $\$ 634,891$ or $6.43 \%$ of allowable costs, and significant in-kind contributions from the air-conditioning and refrigeration industry.

DISTRIBUTION OF THIS DOCUMENT IS UNLIMTEED

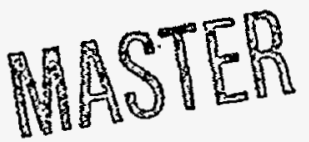




\section{DISCLAIMER.}

The U.S. Department of Energy.'s and the air-conditioning industry's support for the Materials Compatibility and Lubricants Research (MCLR) program does not constitute an endorsement by the U.S. Department of Energy, nor by the air-conditioning and refrigeration industry, of the views expressed herein.

\section{NOTICE}

This report was prepared on account of work sponsored by the United States Government. Neither the United States Government, nor the Department of Energy, nor the AirConditioning and Refrigeration Technology Institute, nor any of their employees, nor any of their contractors, subcontractors, or their employees, makes any warranty, expressed or implied, or assumes any legal liability or responsibility for the accuracy, completeness, or usefulness of any information, apparatus, product or process disclosed or represents that its use would not infringe privately-owned rights.

\section{COPYRIGHT NOTICE}

By acceptance of this article the publisher and/or recipient acknowledges the rights of the U.S. Government and the Air-Conditioning and Refrigeration Technology Institute, Inc. (ARTI) to retain a nonexclusive, royalty-free license in and to any copyright covering this paper. 


\section{DISCLAIMER}

Portions of this document may be illegible in electronic image products. Images are produced from the best available original document. 


\section{TABLE OF CONTENTS}

ABSTRACT

SCOPE

COMPLIANCE WITH AGREEMENT

PRINCIPAL INVESTIGATOR'S EFFORT

\section{SIGNIFICANT RESULTS}

\section{Summary of On-Going Projects}

Thermophysical Properties of HCFC Alternatives.

Sealed Tube Comparisons of the Compatibility of Desiccants with Refrigerants and Lubricants.

Compatibility of Manufacturing Process Fluids with

HFC Refrigerants and Ester Lubricants.

Compatibility of Motor Materials Used in Air-Conditioning for Retrofits

with Alternative Refrigerants and Lubricants.

Products of Motor Burnouts

Accelerated Test Methods for Predicting the Life of Motor Materials

Exposed to Refrigerant-Lubricant Mixtures

Accelerated Screening Methods for Determining Chemical and Thermal

Stability in Refrigerant-Lubricant Mixtures.

Investigation of Flushing and Clean-out Methods

to Ensure System Compatibility.

Investigation Into the Fractionation of Refrigerant Blends

Methods Development for Measuring and Classifying Flammability/Combustibility. of Refrigerants.

Lean Flammability Limits as a Fundamental Refrigerant Property.

Compatibility of Lubricant Additives with

HFC Refrigerants and Synthetic Lubricants.

Study of Foaming Characteristics.

Study of Lubricant Circulation in Systems.

Evaluation of HFC-245ca for Commercial Use in Low Pressure Chillers.

Refrigerant Database 


\section{Summary of Completed Projects}

Thermophysical Properties of HFC-32, HCFC-123, HCFC-124 and HFC-125...... 19-1

Thermophysical Properties of HFC-143a and HFC-152a........................... 20-1

Theoretical Evaluations of R-22 Alternative Fluids .............................. 21-1

Chemical and Thermal Stability

of Refrigerant-Lubricant Mixtures with Metals..................................... 22-1

Miscibility of Lubricants with Refrigerants ........................................ 23-1

Viscosity, Solubility, and Density Measurements

of Refrigerant-Lubricant Mixtures

Measurement of Viscosity, Density and Gas Solubility of Refrigerant

Azeotropes and Blends In Selected Refrigerant Lubricants. ...

Compatibility of Refrigerants and Lubricants

with Motor Materials

Compatibility of Refrigerants and Lubricants with Elastomers

Compatibility of Refrigerants and Lubricants with Engineering Plastics

Electrohydrodynamic (EHD) Enhancement of Pool and In-Tube Boiling

of Alternative Refrigerants

Accelerated Screening Methods for Predicting Lubricant Performance in Refrigerant Compressors. 
DOE/CE/23810-59

\title{
MATERIALS COMPATIBILITY AND LUBRICANT RESEARCH ON CFC-REFRIGERANT SUBSTITUTES
}

\begin{abstract}
The Materials Compatibility and Lubricants Research (MCLR) program supports critical research to accelerate the introduction of CFC and HCFC refrigerant substitutes. The MCLR program addresses refrigerant and lubricant properties and materials compatibility. The primary elements of the work include data collection and dissemination, materials compatibility testing, and methods development. The work is guided by an Advisory Committee consisting of technical experts from the refrigeration and air-conditioning industry and government agencies. The AirConditioning and Refrigeration Technology Institite, Inc., (ARTI) manages and contracts multiple research projects and a data collection and dissemination effort. Detailed results from these projects are reported in technical reports prepared by each subcontractor.
\end{abstract}

\section{SCOPE}

The Materials Compatibility and Lubricant Research (MCLR) program is a multi-year research grant administered by the Air-Conditioning and Refrigeration Technology Institute (ARTI), a notfor-profit organization for scientific research in the public interest. The program was implemented on 30 September 1991 and, as currently funded, will run through 30 September 1996. The MCLR program consists of a number of research projects grouped in phases corresponding to incremental funding periods. The first phase encompassed seven research projects and a data collection and dissemination project. Phase I projects began in January 1992 and have all been completed. Phase II consists of eight research projects and a data collection and dissemination project. Phase II projects began in October 1992 and are currently nearing completion. Phase III consists of ten projects which began in November 1993 and will run through September 1995. Phase IV was approved by the Department on Energy on 15 September 1994. Fifteen research projects and continuation of the data dissemination project are planned for this phase which will run through September 1996.

-This report summarizes the research conducted during the 1st quarter of calendar year 1995. It supersedes the following report numbers:

DOE/CE/23810-48, DOE/CE/23810-42, DOE/CE/23810-38, DOE/CE/23810-33,

$\mathrm{DOE} / \mathrm{CE} / 23810-22, \mathrm{DOE} / \mathrm{CE} / 23810-20$, DOE/CE/23810-11, DOE/CE/23810-8, DOE/CE/23810-4, DOE/CE/23810-3, DOE/CE/23810-2 and DOE/CE/23810-1. 


\section{COMPLIANCE WITH AGREEMENT}

ARTI has complied with all terms of the grant agreement during the reported period.

\section{PRINCIPAL INVESTIGATOR'S} EFFORT

Mr. Mark Menzer is the ARTI principal investigator for the MCLR program. During the fourth quarter of calendar year 1994, Mr. Menzer devoted a total of 121 hours (27.9\% of his available work hours) on the MCLR program. 


\section{THERMOPHYSICAL PROPERTIES OF HCFC ALTERNATIVES}

\section{Objective:}

To provide highly accurate, selected measurements of thermophysical properties to determine equation of state mixture interaction parameters for refrigerant blends and pure fluids. This project will measure primary thermophysical properties of the following refrigerants and refrigerant mixtures:

$\begin{array}{ll}\text { Pure Fluid: } & \text { R-41 } \\ \text { Binary Mixtures: } & \text { R125/134a } \\ & \text { R32/143a } \\ & \text { R125/143a } \\ & \text { R143a/134a } \\ & \text { R32/290 } \\ & \text { R125/290 } \\ & \text { R290/134a } \\ & \text { R41/744 } \\ & \text { R32/125/134a }\end{array}$

These data will consist of simultaneous measurements of the coexisting liquid and vapor densities and compositions as well as the bubble point pressures over a wide range of temperatures and compositions. These data will be used to determine mixing parameters for the Carnahan-Starling-DeSantis (CSD) equation of state and the extended corresponding states (ECS) model.

Results:

The Thermophysical Division, National Institute of Standards and Technology, Boulder, $\mathrm{CO}$, is conducting this project under contract to ARTI. Quarterly technical report, DOE/CE/23810-59A, Thermodynamic Properties of HCFC Alternatives, by W. H. Haynes, April 1995, tabulates coexisting density and bubble point pressure data for $\mathrm{R} 32 / 290, \mathrm{R} 134 \mathrm{a} / 290, \mathrm{R} 125 / 290, \mathrm{R} 32 / 134 \mathrm{a}$ and R32/125 mixtures (Appendix A - Tables 1 through 5, respectively). The data on $\mathrm{R} 32 / 134 \mathrm{a}$ and $\mathrm{R} 32 / 125$ mixtures represent final revised data for these binary systems and replace the preliminary data which were previously reported in the quarterly report, DOE/CE/23810-51A.

Quarterly report, DOE/CE/23810-59A, also compared the R32/134a data with data from the International Energy Agency (IEA) Annex 18 database using a preliminary extended corresponding states (ECS) model to intercompare the data sets. 


\section{SEALED TUBE COMPARISONS OF THE COMPATIBILITY OF DESICCANTS WITH REFRIGERANTS AND LUBRICANTS}

\section{Objectives:}

To provide compatibility information for use of desiccants with potential substitutes for $\mathrm{CFC}$ refrigerants and suitable lubricants.

To obtain data on chemical and thermal stability of desiccants exposed to refrigerantlubricant mixtures under anticipated operating conditions

\section{Results:}

Spauschus Associates, Inc., is performing this research under a contract with ARTI. A detailed report of the progress is contained in the draft final report, DOE/CE/23810-54, Sealed Tube Comparisons of the Compatibility of Desiccants with Refrigerants and Lubricants, by Jay E. Field, PhD.

The research project is to determine the compatibility of sixteen desiccants in thirteen refrigerant-lubricant mixtures using bench-scale sealed tube tests. Samples will be obtained from two manufacturers for each of the eight types of test desiccants.

Test Desiccants:

- $4 \AA \AA$ molecular sieve desiccant beads

- $3 \AA$ molecular sieve desiccant beads

- alumina desiccant

- silica gel desiccant

- core-type desiccant with carbon

10 to $25 \% 3 \AA$ molecular sieve

alumina

5 to $15 \%$ carbon

10 to $20 \%$ phosphate binder

- core-type desiccant with carbon

10 to $25 \% 4 \AA$ molecular sieve

alumina

5 to $15 \%$ carbon

10 to $20 \%$ phosphate binder

- core-type desiccant without carbon

10 to $303 \AA$ molecular sieve

alumina

10 to $20 \%$ phosphate binder 
- core-type desiccant with carbon

10 to $30 \% 4 \AA$ molecular sieve

alumina

10 to $20 \%$ phosphate binder

Test Refrigerant-Lubricant Mixtures and Aging Temperatures:

: R-11 with naphthenic mineral oil @ $105^{\circ} \mathrm{C}$

- R-12 with naphthenic mineral oil @ $149^{\circ} \mathrm{C}$

- R-22 with naphthenic mineral oil @ $149^{\circ} \mathrm{C}$

- R-123 with naphthenic mineral oil @ $105^{\circ} \mathrm{C}$

- R-134a with pentaerythritol mixed-acid polyolester lubricant @ $149^{\circ} \mathrm{C}$

- R-134a with pentaerythritol branched-acid polyolester lubricant @149 ${ }^{\circ} \mathrm{C}$

- R-152a with alkylbenzene lubricant @ 149 $\mathrm{C}$

- R-32 with pentaerythritol mixed-acid polyolester lubricant @ $149^{\circ} \mathrm{C}$

- R-32 with pentaerythritol branched-acid polyolester lubricant @149 $\mathrm{C}$

- R-124 with alkylbenzene lubricant @ $149^{\circ} \mathrm{C}$

- R-125 with pentaerythritol mixed-acid polyolester lubricant @ $149^{\circ} \mathrm{C}$

- R-125 with pentaerythritol branched-acid polyolester lubricant @ $149^{\circ} \mathrm{C}$

- R-143a with pentaerythritol branched-acid polyolester lubricant @ $149^{\circ} \mathrm{C}$

The desiccants were tested by exposure to refrigerant-lubricant mixtures in sealed glass tubes in accordance with ASHRAE/ANSI Standard 97-1989. The desiccants were activated by heating them at $260^{\circ} \mathrm{C}$ for 4 hours prior to sealing in the glass tubes. The following tests or measurements were conducted on unexposed desiccant samples and on desiccant samples after aging with refrigerant-lubricant mixtures and metal catalysts in sealed glass tubes for 28 days:

- visual inspection

- desiccant crush strength

- $\mathrm{GC}$ analysis to determine $\%$ refrigerant decomposed

- total acid number of lubricant

- liquid phase halide ion/acid anion level

- desiccant halide ion/acid anion level

Results are reported by Dr. Field are summarized in the Tables 2-1. through 2-16. 
Liquid Color

Colors follow ASTM Standard D1500.

However, $8 \mathrm{~mm}$ internal diameter is much less than that specified. Therefore, colors "0" through "2" appear the same. The first number listed is the color before aging and the second number is the color after aging.

2.0 water clear

2.5 very faint yellow

3.0 pale yellow

3.5 light yellow

4.0 yellow

4.5 yellow-orange

5.0 light orange

5.5 orange

6.0 orange-brown

6.5 brown

7.0 dark brown

7.5 brown-black

8.0 black

\section{Desiccant Color}

0 no change

1 darker

2 very dark

3 black

Copper Plating

0 none

1 spots on edges

2 edges covered

3 spots on surface

4 partially coated surface

5 fully coated surface

\section{Solids Formation}

0 none

1 small amount

2 medium amount

3 heavy amount $\underline{\text { Steel Corrosion }}$

0 none

1 spot darkening

2 complete darkening

3 pitting or coating

\section{Crush Strength}

The value entered is the average

crush strength in pounds.

GC \% Refrigerant Reacted

Based on peak area ratios for largest

decomposition product detected.

Total Acid Number

$\mathrm{mg}$ of $\mathrm{KOH}$ per gram of oil.

F ion in Liquid

The ppm by weight for the concentration of $F$ ion in the

liquid phase from the aged tube.

\section{F ion on Desiccant}

ppm based on weight of desiccant.

\section{$\mathrm{Cl}$ ion in Liquid}

The ppm by weight for the concentration of $\mathrm{Cl}$ ion in the liquid phase from the aged tube.

Cl ion on Desiccant

ppm based on weight of desiccant.

Organic Acid in Liquid

Sum of the ppm results for all organic anions found in the liquid phase from the aged tube.

Organic Acid on Desiccant

Sum of the ppm results for all organic anions found based on the desiccant weight. 
Table 3-1. Desiccant A: $4 \AA$ Molecular Sieve

\begin{tabular}{|c|c|c|c|c|c|c|c|c|c|c|c|c|c|c|c|}
\hline de & System Fiulds & $\begin{array}{l}\text { Landed } \\
\text { color } \\
\text { est }\end{array}$ & $\begin{array}{l}\text { Dest } \\
\text { color } \\
\text { pes) }\end{array}$ & $\begin{array}{c}\text { copper } \\
\text { pisting } \\
\text { ponp }\end{array}$ & Formation & $\begin{array}{c}\text { Corroston } \\
\text { (es) }\end{array}$ & \begin{tabular}{|c|} 
Crush \\
strength \\
pose
\end{tabular} & $\begin{array}{l}\text { GCX Rel } \\
\text { Rescted } \\
\text { m } x \text { ) }\end{array}$ & 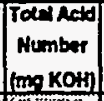 & $\begin{array}{l}\text { Flonh } \\
\text { Lquest } \\
\text { (epm) }\end{array}$ & $\begin{array}{c}\text { Flonon } \\
\text { Desicemx } \\
\text { (ppom) }\end{array}$ & $\begin{array}{l}\text { Cilonh } \\
\text { Uquid } \\
\text { (ppm) }\end{array}$ & 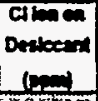 & 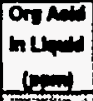 & 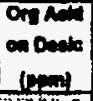 \\
\hline & & 25 & & & & & 67.3 & 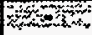 & & & 190 & & als: & 5 & 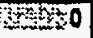 \\
\hline $11=$ & Bitifit & & & & & & 93 & 00 & (0) & & 3130 & 63 & 140 & th & 24 \\
\hline 12 & R12m & 4.0 & & & & & 13.3 & 0.00 & $<0.1$ & 4 & $670^{\circ}$ & 28 & 1.560 & 4 & 570 \\
\hline : & $k p$ & 35 & & & & & $x=12,0$ & 000 & 80 & 6 & 3,60 & $3 x+2$ & $550,600^{\circ}$ & $=0$ & $7>30^{\circ}$ \\
\hline A.14 & R123 & 4.5 & & & & & 16,7 & 0.88 & $\infty .1$ & 1 & 230 & & 720 & 4 & \\
\hline & $d \%$ & 2 & & & & & 10 & $\$ 000$ & 06 & $\mathrm{~b}$ & tES & $\sqrt{2+20}$ & $x=0$ & 2310 & $89 ? 20$ \\
\hline 16 & chededer & 2.5 & & & & & 17.4 & 0.00 & 0.4 & $D$ & 92 & 3 & 32 & 1220 & 6.090 \\
\hline & bivans in & & & & & & 8.7 & 124 & 60.1 & & $3: 140$ & $30^{\circ}$ & 0 & $2,210^{\circ}$ & $=1,300$ \\
\hline & Rasmb & 3.0 & & & & & 92 & 0.55 & 5.6 & 4 & 5,340 & & & 11,630 & 6,070 \\
\hline & hef & & & & & & $\Rightarrow 0$ & 00 & $x_{3}+4+2$ & & $-6,960$ & 6 & 的得: & $4,6,60^{\circ}$ & 73,800 \\
\hline & 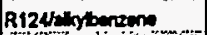 & 3.0 & & & & & 15,6 & 0.41 & $\infty .1$ & 1 & 160 & 9 & 120 & 6 & 3,070 \\
\hline & 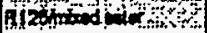 & 2 & & & & & 2123 & 000 & $x=0.1$ & & $3=130$ & 34 & Ex> & 3140 & 11,500 \\
\hline 22 & $\cos _{10 x}$ & 2.5 & & & & & 12.6 & 0.00 & $\begin{array}{l}0.1 \\
0 \\
x_{0}\end{array}$ & & $\begin{array}{r}100 \\
\times \\
\end{array}$ & ${ }_{2}^{3}$ & 10 & $\begin{array}{r}730 \\
x, y 0\end{array}$ & $\begin{array}{r}8,080 \\
40.080\end{array}$ \\
\hline & Bis & & & & & & 124 & 0,00 & 0,3 & lo. & 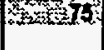 & $y$ & 5 & 40 & 28,610 \\
\hline & 100 & & & & & & 94.0 & 0.00 & $=0,5$ & & 330 & 64 & $2 ; 30$ & $=16$ & $=66^{\circ}$ \\
\hline & R12trit & 4.0 & & & & & 13.1 & 0.00 & 0.1 & 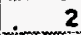 & 1.240 & 21 & 1,390 & 10 & 820 \\
\hline & (10) & 3.5 & & & & & 204 & $=0,0$ & 801 & $z=12$ & $3=3,940$ & $=670$ & 31700 & 0 & 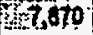 \\
\hline 44. & R123hinerd od & 4.5 & & & & & 14.6 & 0.68 & $<0.1$ & & 260 & & 840 & & \\
\hline & tow & 2,3 & & & & & $8 \mathrm{f}$ & 0.00 & a.s. & & $3=6$ & $=3 x$ & Wit & 91950 & 8,680 \\
\hline & chededest & 2.5 & & & & & 12.8 & 0.00 & & & & & & 430 & 6,390 \\
\hline & $\cos ^{n}=x$ & (3.0) & & & & & 28 & $y=1.30$ & 0 & i & 3,50 & 2800 & 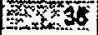 & 2,060 & $3 ; 10$ \\
\hline 48 & R32 froud derer & 3.0 & & & & & 8.8 & 0.42 & 4.6 & 0 & 5,840 & 3 & 18 & 12410 & 6,800 \\
\hline & $R$ & 25 & & & & & 77.2 & 0.40 & $=1.4$ & 6 & 18,130 & $200^{\circ}$ & $5<32$ & 13360. & 17,900 \\
\hline .50 & R124hostomeno & 3.0 & & & & & 14.4 & 0.35 & $<0.1$ & 1 & 280 & 11 & 110 & & 2,860 \\
\hline & & 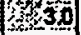 & 30 & & 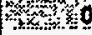 & & 43 & 0 & 0.1 & 28 & 260 & $6=6$ & Dren 25 & 2400 & $=0,700$ \\
\hline & renched ener & 2.5 & & & 0 & & $\frac{11.0}{110}$ & 0.00 & 0.1 & 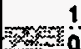 & 110 & 6 & 17 & $\begin{array}{r}1300 \\
x \\
3\end{array}$ & 9,000 \\
\hline & & & & & & & & & & & & & & & \\
\hline
\end{tabular}

Table 3-2. Desiccant E: $4 \AA$ Molecular Sieve

\begin{tabular}{|c|c|c|c|c|c|c|c|c|c|c|c|c|c|c|c|}
\hline cosels & Bypten flude & $\begin{array}{l}\text { Wquid } \\
\text { color } \\
(20)\end{array}$ & $\begin{array}{l}\text { Deste } \\
\text { color } \\
0,31\end{array}$ & \begin{tabular}{|c|} 
Copper \\
Plisting \\
o-s
\end{tabular} & \begin{tabular}{c|} 
Solds: \\
Formution \\
(2)
\end{tabular} & $\begin{array}{c}\text { Corrostion } \\
\text { p.s) }\end{array}$ & $\begin{array}{c}\text { Cruth } \\
\text { strongth } \\
\text { pers }\end{array}$ & 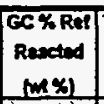 & 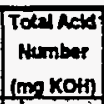 & $\begin{array}{l}\text { Flonh } \\
\text { Lquid } \\
\text { (pem) }\end{array}$ & $\begin{array}{c}\text { Flonon } \\
\text { Destecem } \\
\text { (pem) }\end{array}$ & 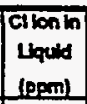 & $\begin{array}{c}\text { Cilonion } \\
\text { Dociceman } \\
\text { (ppony }\end{array}$ & 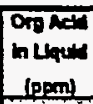 & $\begin{array}{l}\text { ordadd } \\
\text { on Doste } \\
\text { (pen) }\end{array}$ \\
\hline New & Honiti: & 25 & & & & & $5 i=309$ & 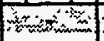 & & & 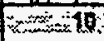 & & at & 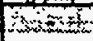 & misciso \\
\hline 110 & Rithi & $3_{3}^{3} 4$ & & & & & 122 & 00,0 & 20 & & $9 x+130$ & $1=\pi 10$ & 7,570 & 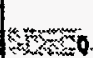 & 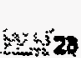 \\
\hline 2 . & $\mathrm{R} 12 \mathrm{~m}$ & & & & & & 27.0 & 0.00 & $<0.1$ & & 40 & & 680 & & 272 \\
\hline 3 & Aotion & (a) & & & & & 27. & 0,12 & 60. & & $6,230:$ & 340 & 0,000 & 50 & $\theta, 920$ \\
\hline & R123h & 3.5 & & & & & $35.6]$ & 0.00 & $<0.1$ & & 54: & & & $i$ & 12 \\
\hline & Biat: & 40 & & & & & 210 & 3.00 & $\infty$ & & 80 & & $2+4$ & 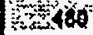 & 2,130 \\
\hline & Risen & 2.5 & & & & & 23.2 & 0.00 & $<.1$ & & 630 & & 64 & 89 & 4,280 \\
\hline & (11020 & 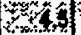 & & & & & 23,7 & isina & 30 & & 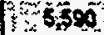 & 40 & & 10330 & 31,290 \\
\hline f & $832 \mathrm{~mm}$ & 3.0 & & & & & 21.0 & 0.35 & 4.7 & 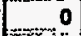 & 5.270 & 0 & 0 & 10,000 & 5,170 \\
\hline & Roth & 24 & & & & & 177 & $|x=0.40|$ & 政 & & 87.10 & & $2=0$ & 70 & 18,700 \\
\hline f & R12U: & 2.5 & & & & & 25.2 & $\begin{array}{l}0.16 \\
-0.00\end{array}$ & $<0.1$ & & 83 & 6 & 43 & 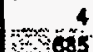 & 24 \\
\hline $\begin{array}{ll}E-21 \\
E-22\end{array}$ & $\begin{array}{l}\text { H125h } \\
\text { Rizst }\end{array}$ & $\begin{array}{l}2.35 \\
2.5\end{array}$ & & & & & $\begin{array}{r}22.0 \\
25.5\end{array}$ & 0.000 & $\begin{array}{r}8.1 \\
<0.1\end{array}$ & t: & & $\frac{5}{2}$ & $\begin{array}{r}57 \\
13\end{array}$ & $\begin{array}{r}0005 \\
100\end{array}$ & $\begin{array}{l}1,250 \\
1,220\end{array}$ \\
\hline$E \div 23$ & $a_{m}^{2}$ & in & & & 이 & & rine & 00,000 & 02 & 50 & $p t s=7$ & $|x|$ & 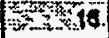 & 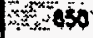 & as $=60$ \\
\hline & & & & & & & 01 & 80 & & & 20 & & & & \\
\hline 2 & R12 & 3.0 & & & & & 23.6 & 0.00 & $<0.1$ & & 570 & & & 19 & \\
\hline in & perth & rito & & & & & 33.0 & 0,0 & 60, & it & 5,620 & 87 & stsop. & & 10530 \\
\hline 4.7 & Ri12zm & 3.0 & & & & & 27.7 & $0.2 ?$ & 0.7 & & & 20 & 490 & & \\
\hline it & Hisis & 25 & & & & & 25,8 & 0.00 & 02 & & $=2,84$ & & 30 & x 30 & $=1,600$ \\
\hline E-46 & tind eater & 25 & & & & & 23.0 & 0.00 & 0.3 & & $\begin{array}{r}28 \\
6606\end{array}$ & 6 & 10 & $\begin{array}{r}350 \\
2570\end{array}$ & $\begin{array}{r}510 \\
6160\end{array}$ \\
\hline$E-48$ & 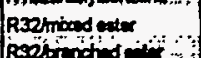 & 3.0 & & & & & $\begin{array}{r}17.2 \\
18.7\end{array}$ & 0.46 & $\begin{array}{l}5.1 \\
4.6\end{array}$ & & $\begin{array}{r}4.970 \\
r=7000\end{array}$ & $\rightarrow 0$ & $\begin{array}{l}0 \\
3=0\end{array}$ & $\begin{array}{r}14,260 \\
77330\end{array}$ & $\begin{array}{r}5,130 \\
0.250\end{array}$ \\
\hline$E .50$ & Ri2u & 2.5 & & & & & 30.6 & 0.29 & $<.1$ & & & 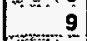 & & & 200 \\
\hline & & 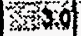 & & & & & 26.3 & 00.00 & 0,4 & & & & काष & 230 & $\$, 400$ \\
\hline & & 2.5 & & & & & 2.9 & 0.13 & 0.1 & 0 & ini & & & 180 & 120 \\
\hline & & 23 & & & & & & 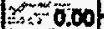 & 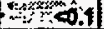 & & & & tots. & $2 x+217$ & $x+5020$ \\
\hline
\end{tabular}


Table 3-4. Desiccant F: $3 \AA$ Molecular Sieve

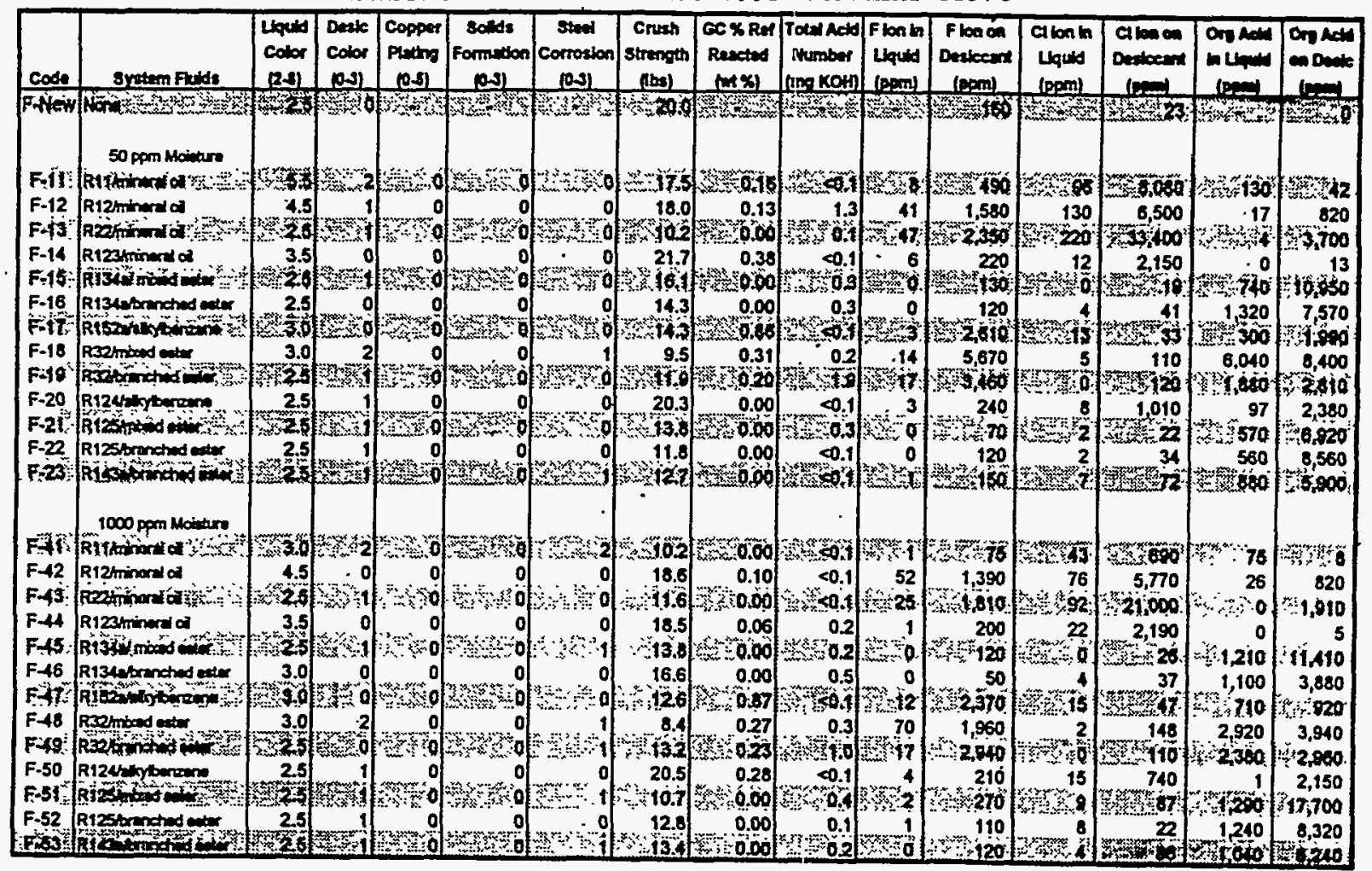

Table 3-4. Desiccant H: $3 \AA$ Molecular Sieve

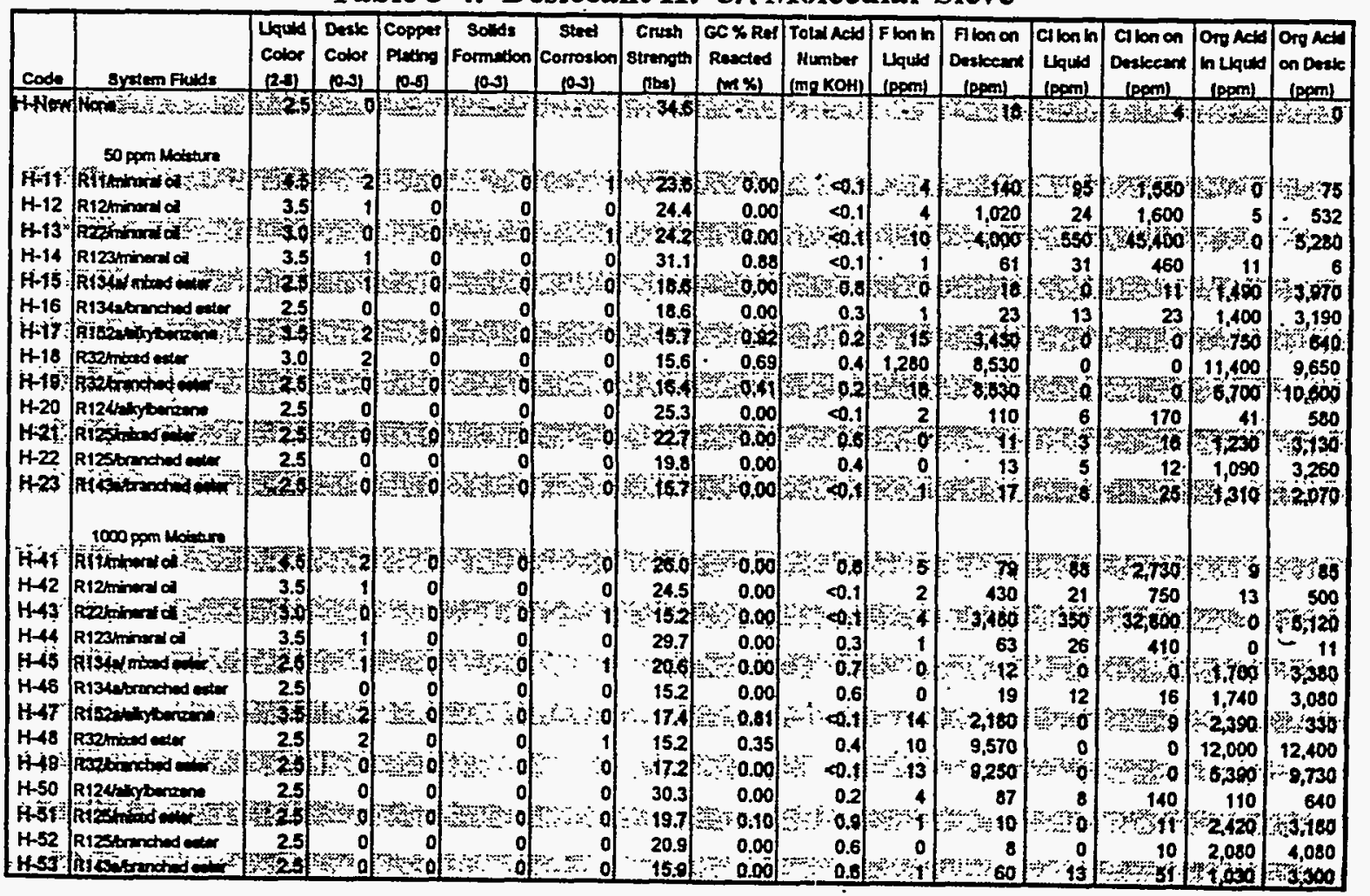


Table 3-5. Desiccant I: Alumina

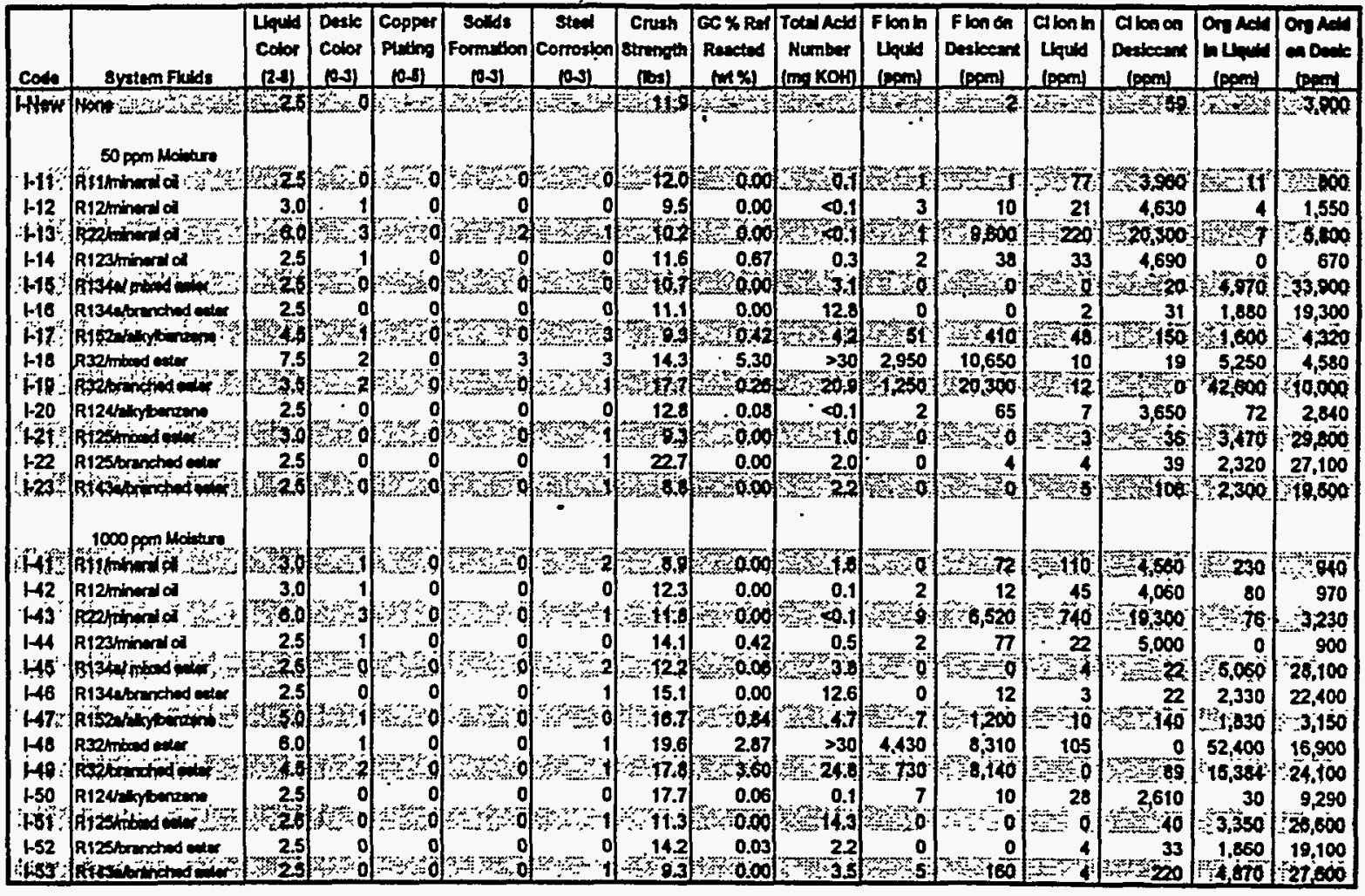

Table 3-6. Desiccant J: Alumina

\begin{tabular}{|c|c|c|c|c|c|c|c|c|c|c|c|c|c|c|c|}
\hline ode & Syztem Fulds & \begin{tabular}{|l|} 
Lquid \\
Color \\
$(2+1)$ \\
\end{tabular} & $\begin{array}{l}\text { Deale } \\
\text { Color } \\
\text { pesl }\end{array}$ & $\begin{array}{l}\text { coppert } \\
\text { pesting } \\
\text { pers }\end{array}$ & $\begin{array}{c}\text { Formestion } \\
\text { pes! }\end{array}$ & $\begin{array}{c}\text { atcet } \\
\text { Corroston } \\
\text { pes }\end{array}$ & \begin{tabular}{|c|} 
Crusth \\
strongth \\
(ibel
\end{tabular} & $\begin{array}{c}G C \% R \operatorname{Rof} \\
\text { Receted } \\
\ln (x)\end{array}$ & 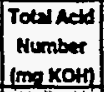 & $\begin{array}{l}\text { Flon hn } \\
\text { Unqued } \\
\text { (pen) }\end{array}$ & 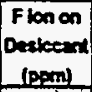 & $\begin{array}{l}\text { Allon h } \\
\text { Llqueld } \\
\text { Lepen! }\end{array}$ & $\begin{array}{l}\text { Criton on } \\
\text { Desikeant } \\
\text { (penn) }\end{array}$ & 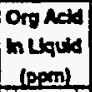 & $\begin{array}{c}\text { org Actd } \\
\text { on Dente } \\
\text { (Bem) }\end{array}$ \\
\hline 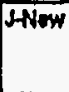 & Horn & & & & & & $2 \pi$ & & & & 5 & 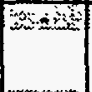 & as 10 & Lints & 5.155 \\
\hline 1 & Ritimin & 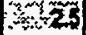 & & & & & 22,3 & 0.00 & 管 & 勧 & 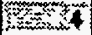 & 46 & 3,120 & 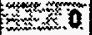 & 600 \\
\hline & $R 12 \min$ & & & & & & 33.4 & 0.00 & $<0.1$ & 3 & 64 & & 3,910 & 250 & 690 \\
\hline & Abn & & & & & & $62 \%$ & 0,00 & 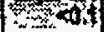 & 16 & 8680 & 60 & 14,300 & 200 & 0,070 \\
\hline & R123hineral all & & & & & & 20.7 & 0.48 & $<0.1$ & 2 & 51 & 19 & 3,810 & 6 & 120 \\
\hline & Ristw & & & & & & 219 & 0.00 & zit) & & 等 & 3 & zitlt & $-3,000$. & 19,60 \\
\hline & Riscan & & & & & & 19.4 & 0.00 & 1.9 & 01 & 0 & 3. & 14 & 2,580 & 16,300 \\
\hline & R162avil & & & & & & 208 & +43 & 20 & 20 & 3220 & 160 & $2 x$ & 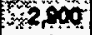 & 2,6 \\
\hline & R32mimbor & .5 & & & & & 25.1 & 1.34 & $>30$ & 4,990 & 4,940 & 59 & 59 & 67,600 & 60,200 \\
\hline & R32/axim & 3. & & & & & 25,8 & 0.00 & 30 & $=1, \infty 60$ & Entonso & 0 & $5=24$ & 515,600 & 12,200 \\
\hline & Ri2w: & & & & & & 31.8 & 0.00 & 0.1 & & & & 2.810 & 17 & $6,6.6$ \\
\hline & B125int & & & & & & 20 & 0.00 & 72 & & 30 & mo & Gxis? & 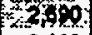 & 15,20 \\
\hline & R125tor & & & & & & 244 & 0.00 & & & & & & 2,110 & 13,3 \\
\hline & Rivisinarex & 201 & & & & & 27,4 & 4000 & 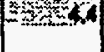 & 0. & $z=0$ & 47 & Waxta & Fist. & 1980 \\
\hline & Rélithon & 20 & & & & & 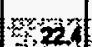 & $3=0.00$ & 0.3 & 52 & 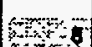 & 34 & 3,310 & 130 & $=470$ \\
\hline & R12tmin & & & & & & 30.5 & 0.00 & $<.1$ & 1 & $\pi$ & 30 & 3,580 & 80 & 80 \\
\hline & heosming & & & & & & 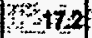 & 0.40 & $\infty .1$ & $\mathbf{5}$ & 4,630 & 720 & 13,300 & 260 & 2730 \\
\hline & R123minamel ad & & & & & & 20.5 & 0.57 & & & $120^{\circ}$ & & 3980 & o. & 210 \\
\hline & lasusis & & & & & & 168 & 0,06 & & 0 & & ?0? & 2 & 5.060 & $22: 10$ \\
\hline & R136tebrenctued ander & & & & & & 23.7 & 0.00 & & & & & & 4,670 & 12,00 \\
\hline & R̂́tăgin & 5.0 & & & & & $-23.5 \mid$ & 0.70 & $=2 \times 20$ & $\pi$ & 420 & $3=0$ & $\geqslant 0$ & 3,280 & 3.25 \\
\hline & R32 Anim & 7.5 & & & & & 25.0 & 1.60 & $>30$ & 4,960 & 4,520 & & & 75,800 & 56,20 \\
\hline & 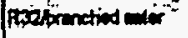 & & & & & & 29.4 & 5.00 & $2 ? 30$ & 1,300 & 11,900 & 00 & 170 & 23,600 & 4,600 \\
\hline & 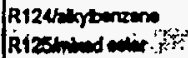 & 2.5 & & & & & $\begin{array}{r}27.9 \\
0\end{array}$ & $\begin{array}{r}0.00 \\
30.00\end{array}$ & $\begin{array}{l}<.1 \\
x \leq 12.7\end{array}$ & 20 & $\begin{array}{r}35 \\
3 x=0\end{array}$ & $\begin{array}{r}7 \\
0 \\
0\end{array}$ & $\begin{array}{r}2,470 \\
1 \\
3\end{array}$ & $\begin{array}{r}93 \\
-7,720 \\
20\end{array}$ & $\begin{array}{l}7,000 \\
20,400\end{array}$ \\
\hline & & $m i$ & & & & & $21: 5$ & sen & & & & & & $\infty$ & 1 \\
\hline & & & & & & & & & & & & & & & \\
\hline
\end{tabular}


Table 3-7. Desiccant K: Silica Gel

\begin{tabular}{|c|c|c|c|c|c|c|c|c|c|c|c|c|c|c|c|}
\hline ods & $8 y$ frods & $\begin{array}{l}\text { laped } \\
\text { color } \\
\text { (2) }\end{array}$ & $\begin{array}{l}\text { Deak } \\
\text { color } \\
\text { pul }\end{array}$ & $\begin{array}{c}\text { Coppos } \\
\text { platho } \\
\text { pas }\end{array}$ & $\begin{array}{l}\text { oolds } \\
\text { formation } \\
\text { (D) }\end{array}$ & $\begin{array}{c}\text { stivi } \\
\text { corroston } \\
0.31\end{array}$ & $\begin{array}{c}\text { Crusth } \\
\text { Strength } \\
\text { (1) }\end{array}$ & 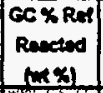 & 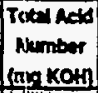 & 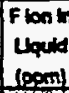 & 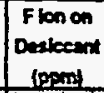 & $\begin{array}{l}\text { Cilon in } \\
\text { Liquld } \\
\text { (pem) }\end{array}$ & $\begin{array}{c}\text { Cilon on } \\
\text { Detikent } \\
\text { (pem }\end{array}$ & 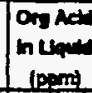 & 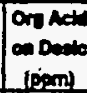 \\
\hline How & 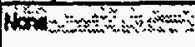 & $2=20$ & & & & 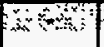 & \$78 & ry & 8 & $x$ & xकीय & & andste & 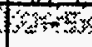 & 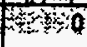 \\
\hline & & & & & & & & & & & & & & & \\
\hline i1: & Rithinguril od & 20 & & & & & 90 & 0.00 & $\infty .1$ & $\sqrt{2}$ & $x \sin 200$ & $6,1,620$ & 40.030 & 00 & 100 \\
\hline 2 & R12hinumal & 2.5 & & & & & 50.8 & 0.00 & $<0.1$ & & & 600 & & 15 & \\
\hline & 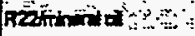 & 23 & & & & & $-6 \$ 9$ & 0.00 & 0.1 & 52 & 1,290 & 330 & 680 & $=0$ & 390 \\
\hline 4 & R123Atinares of & 2.5 & & & & 7 & 68.5 & 0.00 & 0.1 & 2 & 19 & 37 & 230 & 33 & 50 \\
\hline & 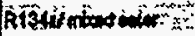 & 28 & & & & & 8 & into & 251 & & 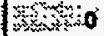 & 80 & 80 & 24,000 & 20,000 \\
\hline 3 & R134etronched eats & 2.5 & & & & & 60.3 & 0.00 & 15.7 & 0 & 0 & 5 & 0 & 15,100 & 11,900 \\
\hline & 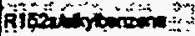 & 29 & & & & & 362 & 0.00 & 00.1 & 6 & 3,800 & & & 700 & $=0$ \\
\hline s. & 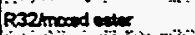 & 2.5 & & & & 7 & 11.9 & 0.24 & 143 & 4 & 11. & 3 & 0 & 22,100 & 11,100 \\
\hline $10^{\circ}$ & Rsorimaton & 25 & & & & & $12=3$ & 0.00 & 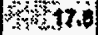 & & W & 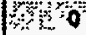 & 40 & 78,600 & 1013,400 \\
\hline 20 & 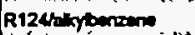 & 2.5 & & & & 2 & 74.1 & 0.00 & $\infty .1$ & 3 & & 17 & 55 & 130 & \\
\hline $1^{\circ}$ & Rizotrind & $x, 30$ & & & & & $=66,9$ & 000 & aitiz & & 0 & 13 & intit & $318 ; 400$ & 24,400 \\
\hline 22 & R12stronched wetur & 2.5 & & & & & 97.9 & 0.00 & 13.6 & $\ddot{0}$ & 22 & & 0 & 19,200 & 14,200 \\
\hline 23 & Af & 28 & & & & & 477 & 30.00 & 8137 & & $x=0$ & $x+3$ & 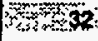 & $1,1,900$ & $8,310^{\circ}$ \\
\hline & & & & & & & & & & & & & & & \\
\hline (ij: & Rith & 30 & & & & & (9) & 0.00 & & & 8010 & 1570 & 28.970 & 42 & 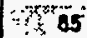 \\
\hline$=$ & R12minewed od & 2.5 & & & & & 86.1 & 0.00 & $<0.1$ & 3 & 360 & 570 & & & \\
\hline 3 & 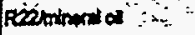 & 28 & & & & & 68.5 & 0.00 & 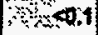 & 45 & 等 & 670 & $x+350$ & $17 \%$ & t450 \\
\hline 44 & Rizatininerd ad & 2.5 & & & & & 45.2 & 0.16 & $<0.1$ & & & & & & \\
\hline 45: & Ríżer maid mix & $2 ; 8$ & & & & & 9.8 & 0 & 16.5 & & $=0$ & $F$ & 811 & 17,600 & 16,600 \\
\hline & R134ubranctiod atax & 3.0 & & & & & 51,6 & 0,00 & 21.6 & & & & 9 & 14,000 & 14,200 \\
\hline & Ristomand & 25 & & & & & 48.5 & 0.05 & $\infty \infty, 1$ & 22 & $4, \quad 3,460$ & $\because 5$ & 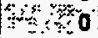 & 7. 830 & 30 \\
\hline & R352triced atur & 2.5 & & & & & 28.0 & 0.22 & 17.1 & 9 & & & 0 & 480 & 14,000 \\
\hline & Resersination in & 26 & & & & & 80.5 & 443 & 18.2 & 110 & $=283$ & 19 & 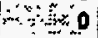 & $-10,900$ & 700 \\
\hline ? & 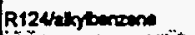 & 25 & & & & & 75.6 & 0.13 & 0.1 & 14 & & & & & \\
\hline & $d=0$ & 27 & & & & & $\Rightarrow 58.0$ & $\mid 2,0,00$ & $28 . \hat{6}$ & & & 10 & $=9$ & $16,000^{3}$ & 96,400 \\
\hline K-5 & & 2.5 & & & & & 65.9 & 0.66 & $>30$ & 0 & & & & 11,500 & 19,100 \\
\hline & & & & & & & & & & & & & & 1190 & 27,100 \\
\hline
\end{tabular}

Table 3-8. Desiccant L: Silica Gel

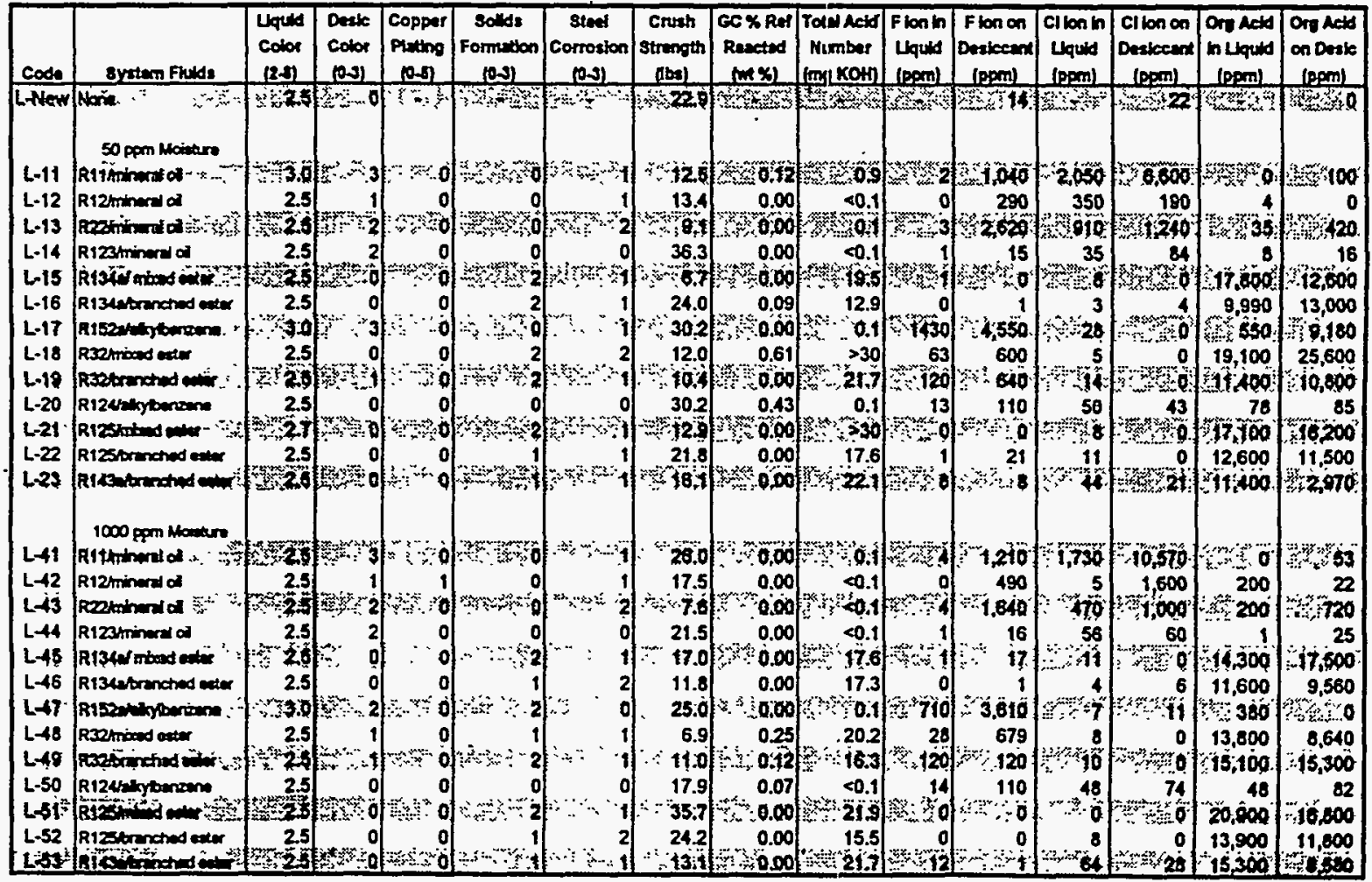


Table 3-9. Desiccant M: $3 \AA$ Carbon Core

\begin{tabular}{|c|c|c|c|c|c|c|c|c|c|c|c|c|c|c|c|}
\hline $\operatorname{cose}$ & Syctem Fuids & $\begin{array}{l}\text { Laids } \\
\text { Color } \\
\text { (2-2) }\end{array}$ & $\begin{array}{l}\text { Deate } \\
\text { Color } \\
\text { pol }\end{array}$ & $\begin{array}{c}\text { Copper } \\
\text { Pisting } \\
\text { pon }\end{array}$ & $\begin{array}{c}\text { Solwd } \\
\text { formeson } \\
\text { (a) }\end{array}$ & $\begin{array}{c}\text { corrostion } \\
\text { (D) }\end{array}$ & $\begin{array}{c}\text { Crush } \\
\text { strength } \\
\text { (thesl }\end{array}$ & 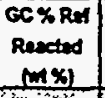 & $\begin{array}{l}\text { Totw Add } \\
\text { Mumber } \\
\text { Imp Kot? }\end{array}$ & $\begin{array}{l}F \text { lon hn } \\
\text { Inquat } \\
\text { (popm) }\end{array}$ & 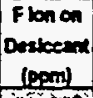 & $\begin{array}{l}\text { Cloon in } \\
\text { Lquat } \\
\text { (ppent) }\end{array}$ & 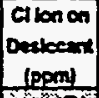 & 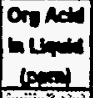 & 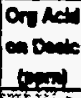 \\
\hline$\overline{\mathrm{Ne}}$ & Nom & 29 & & & & & & 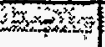 & & & 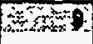 & & Fint & 2 & axtox \\
\hline 18 & (ai) & & & & & & & 00 & & & (33) & 160 & 83101 & sisyo & 160 \\
\hline 12 & 2 2hinere 1 & 3.0 & & & & 1] & 7.3 & 0.09 & $<0.1$ & 5 & 66 & 33 & 3,300 & 3 & 240 \\
\hline & $R 20 \%$ & s.5. & & & & & 4.1 & 0.06 & 60.1 & & 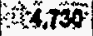 & & 16.700 & $240^{\circ}$ & 2,770 \\
\hline 4 & R1zmin & 2.5 & & & & & 5.5 & 0.34 & 0.5 & 84 & 210 & & 2,740 & 0 & \\
\hline$\overline{6}$ & 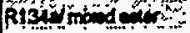 & 25 & & & & & & 0.0 & 34 & 0 & 0 & & & 0,210 & 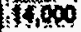 \\
\hline & R134torendred exter & 2.5 & & & & & 8.2 & 0.00 & 3.9 & 24 & 0 & 3 & $x+1$ & 8,620 & 11,700 \\
\hline $\mid \bar{t}:$ & Alo & 10 & & & & & 87 & opon & T.8 & : & 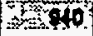 & & 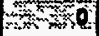 & $2 \%$ & $\$ 70$ \\
\hline & R32thix & 7.0 & & & & & 5.8 & 1.47 & $>30$ & 540 & 9,080 & 5 & 45 & 39,700 & 28,800 \\
\hline 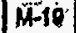 & $y=0$ & 3 & & & & & 47 & $3.7 \mathrm{Q}$ & 830 & 鴊 2,140 & 8.760 & 38 & 30 & $11 ; 000$ & 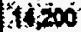 \\
\hline 1 & R12uratyongene & 2.5 & & & & & -6.5 & 0.05 & $\infty .1$ & 8 & 240 & & 1,470 & 10 & 2.880 \\
\hline & 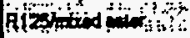 & zol & & & & & 7.0 & 0000 & 50 & 3 & 311 & 86 & $=16$ & 0,070 & 17.609 \\
\hline 22 & Rizstorn & 25 & & & & & & 0.00 & & & & & & 4290 & 16,500 \\
\hline & Rikikin & & & & & & (4) & :2: & & & & & sit & $36<200$ & 10500 \\
\hline & 10 & & & & & & & & & & & & & & \\
\hline 2 & Bitikn & 3.0 & & & & & 6.7 & 0.00 & & & $x=40$ & 130 & 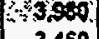 & 我 & $x$ \\
\hline 42 & R12min & 3.0 & & & & & 8.0 & 0.00 & $\begin{array}{c}c 0.1 \\
01\end{array}$ & 18. & 75 & 64 & $\begin{array}{r}3,460 \\
07\end{array}$ & 230 & $\begin{array}{r}1,060 \\
0\end{array}$ \\
\hline & pra & $\begin{array}{l}x .3 \\
2.5\end{array}$ & & & & & & 0.30 & 1.2 & & 160 & & 2,700 & $\begin{array}{r}000 \\
4\end{array}$ & D. \\
\hline & 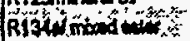 & & & & & & 7.4 & 0.13 & 3.1 & 6 & $9 x=1$ & $3 \frac{3}{3}$ & & $=5,020$ & 15,0 \\
\hline & RI3leprenated exter & 2.5 & & & & & 9.6 & 0.00 & & & & & & 3,510 & 11,400 \\
\hline 7 & 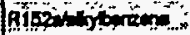 & 4 & & & & & 51 & 20 & 0,7 & $170^{\circ}$ & $=2,300$ & $7 ?$ & $=516$ & A.6je & $1 ; 250$ \\
\hline & R32mbeod notum & 70 & & & & & & 0.24 & $>30$ & 3,280 & 9,980 & 10 & 40 & 26,900 & 27,000 \\
\hline & 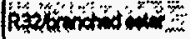 & & & & & & 38 & 20,00 & 330 & $1 ; 690$ & 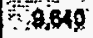 & 50 & $28=38$ & $T 9,000$ & 48,20 \\
\hline 50 . & R124histomane & & & & & & & 0.00 & & & 230 & 14 & 1,730 & & 3,830 \\
\hline & BIIsomd & & & & & & & $=0,0$ & (10.3 & 20 & No & $\stackrel{a}{a}$ & 30 & 0,600 & $\$ 3,0,00$ \\
\hline & $R 125 / 4$ & 25 & & & & & & 0.00 & 9.9 & & & & 20 & 3,830 & tis \\
\hline & & & & & & & & & & & & & & & \\
\hline
\end{tabular}

Table 3-10. Desiccant N: $3 \AA$ Carbon Core

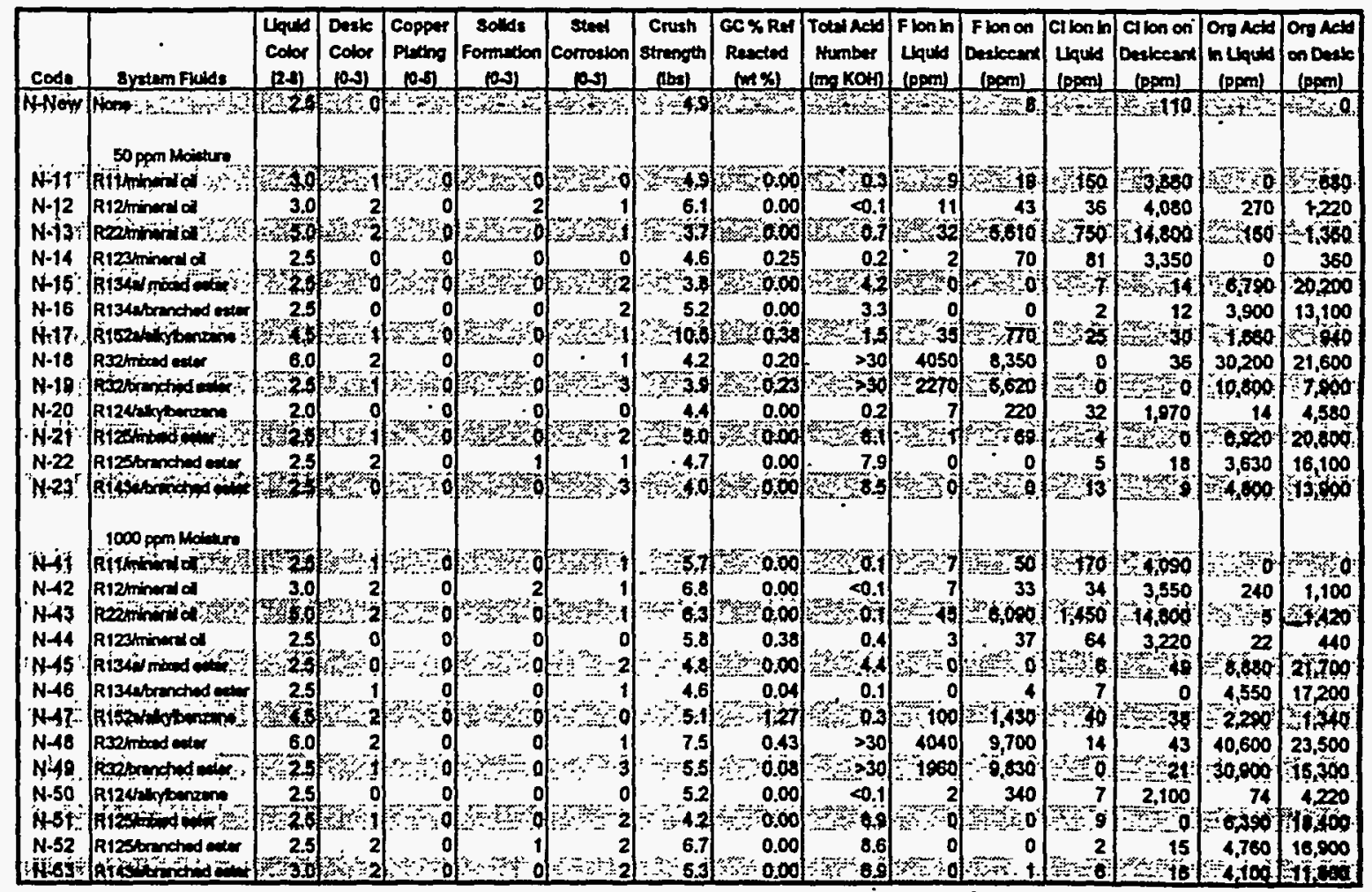


Table 3-11. Desiccant T: $4 \AA$ Carbon Core

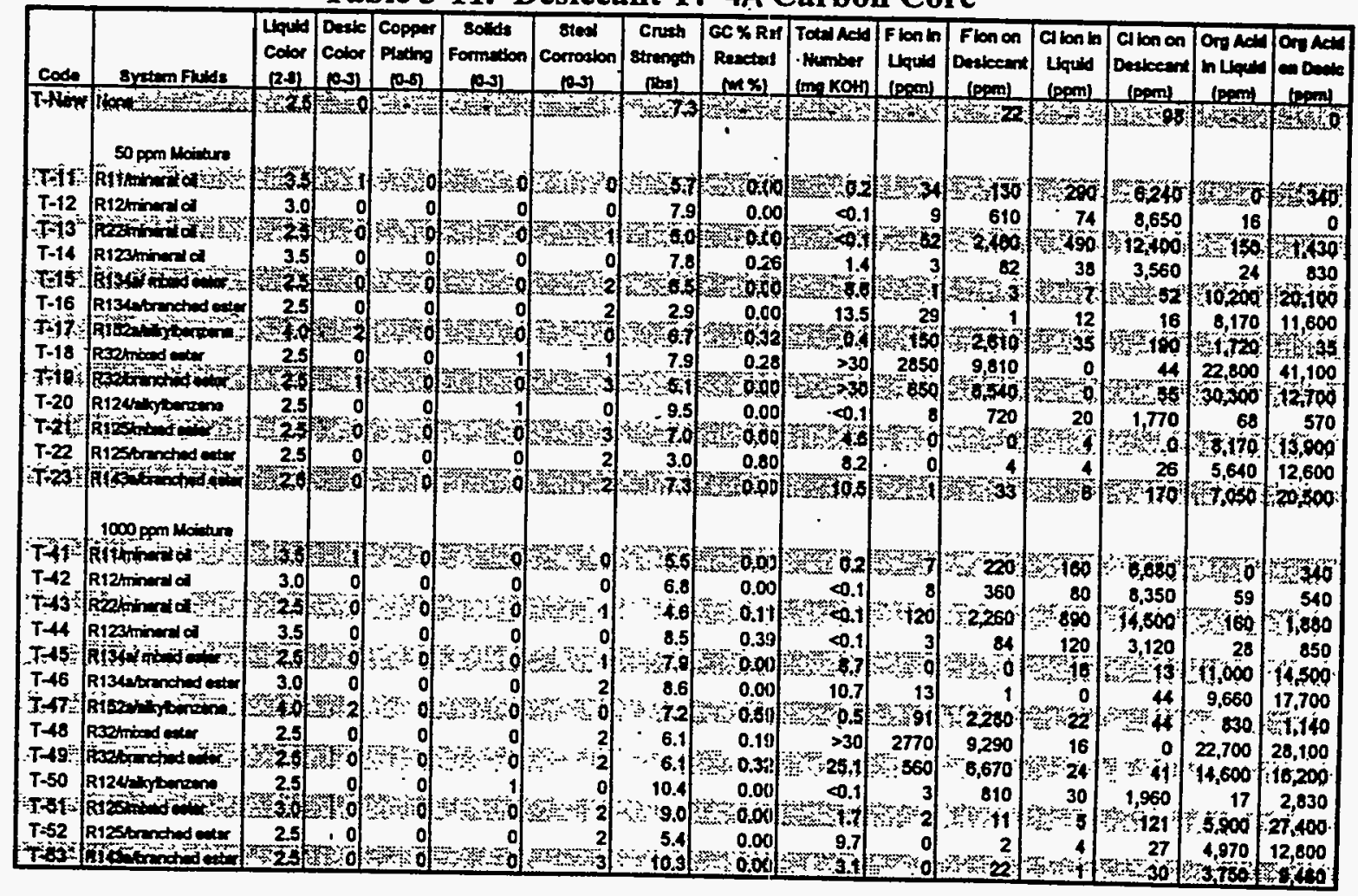

Table 3-12. Desiccant V: $4 \AA$ Carbon Core

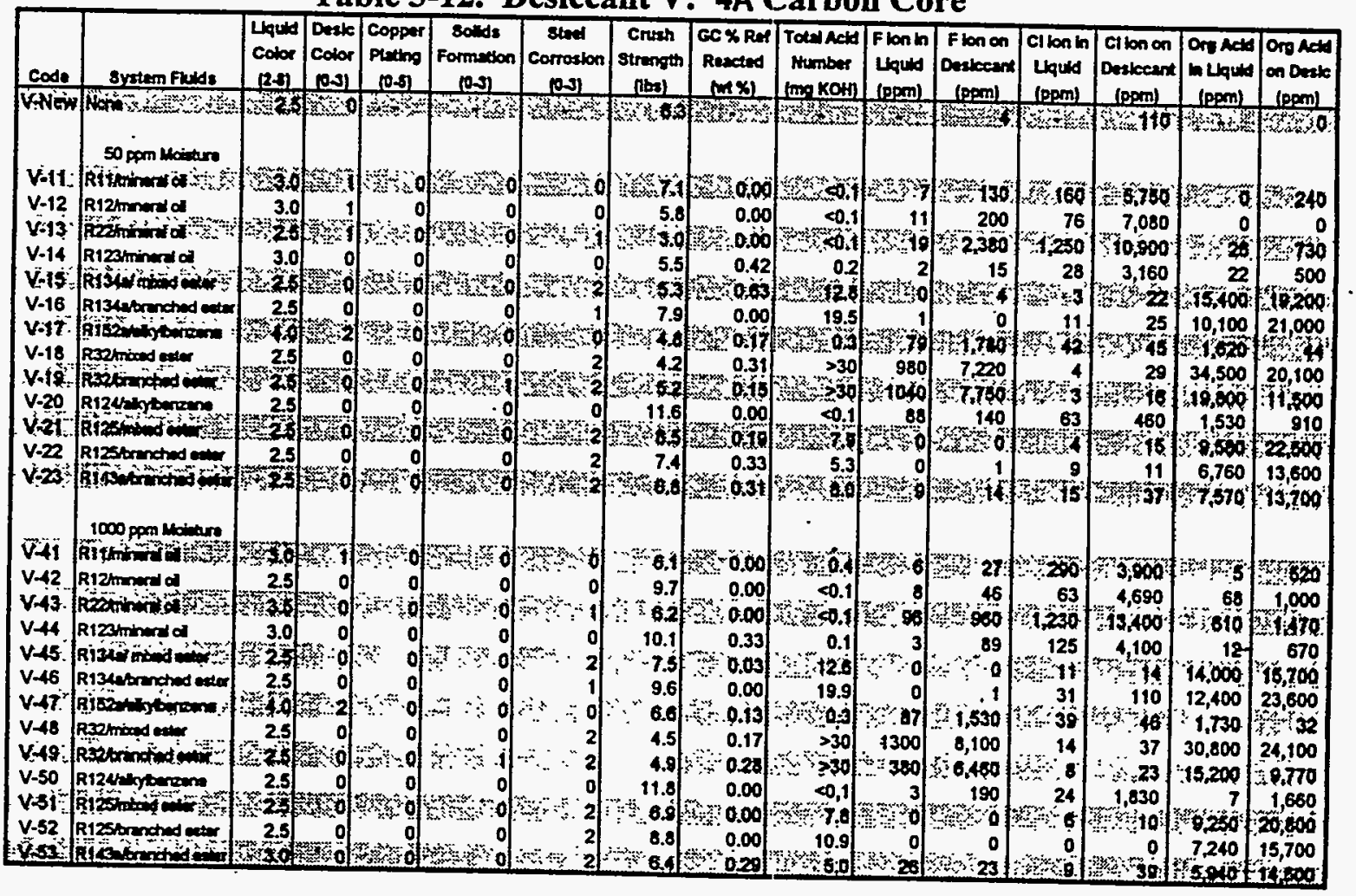


Table 3-13. Desiccant W: $3 \AA$ Core (No Carbon)

\begin{tabular}{|c|c|c|c|c|c|c|c|c|c|c|c|c|c|c|c|}
\hline Cope & Sycten filds & $\begin{array}{l}\text { Lande } \\
\text { color } \\
\text { pol }\end{array}$ & {$\left[\begin{array}{l}\text { Dente } \\
\text { color } \\
0,3\end{array}\right]$} & {$\left[\begin{array}{c}\text { Copper } \\
\text { Piturn } \\
\text { p-sis }\end{array}\right]$} & $\begin{array}{c}\text { sollds } \\
\text { formetion } \\
\text { pul }\end{array}$ & $\begin{array}{c}\text { stmol } \\
\text { corrostion } \\
\text { (-S) }\end{array}$ & $\begin{array}{c}\text { Crush } \\
\text { strength } \\
\text { (bes) }\end{array}$ & 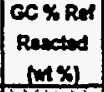 & $\begin{array}{l}\text { Tot lest } \\
\text { Number } \\
\text { Im KOHI) }\end{array}$ & $\begin{array}{l}\text { Flon hn } \\
\text { Laqued } \\
\text { (pesm) }\end{array}$ & 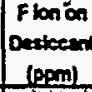 & $\begin{array}{l}\text { Crionin } \\
\text { Lquild } \\
\text { (pent) }\end{array}$ & 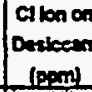 & 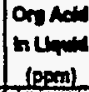 & 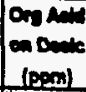 \\
\hline $\mathrm{Nim}$ & 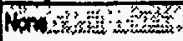 & 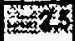 & 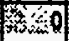 & & 8 & 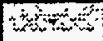 & $6^{2} \leq 40$ & $x+2$ & Fin & $x_{2} z^{2}=$ & 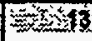 & $4 x+2$ & कx & 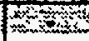 & 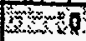 \\
\hline itt: & 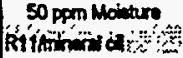 & $2 ; 5$ & & & & & 5.3 & 0,00 & $6=0.1$ & $21+9$ & 0 & $y=110$ & 3,340 & 4023 & 110 \\
\hline 12 & R12hinared a & 3.0 & & & & & 5.4 & 0.00 & $<0.1$ & 8 & & 73 & 3,430 & 1 & \\
\hline 3 & 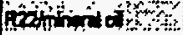 & 3 & & & & & 57 & 0.00 & $\$ 0,1$ & $=18$ & 10,250 & 600 & 10.500 & 4 & 32200 \\
\hline 14 & RI23minert of & 2.5 & & & & & 4.5 & 0.36 & $\$ 0.1$ & & 7 & 75 & 3,840 & 3 & 300 \\
\hline is: & Riativind & 2,8 & & & & 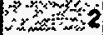 & 7,8 & 00 & $\sqrt{20}$ & mons & $x=0$ & & 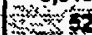 & 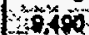 & 1,700 \\
\hline-16 & 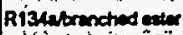 & 2.5 & & & & & & $0 . \infty$ & 8.0 & 0 & & & & 6,770 & 23,100 \\
\hline & Gilopinjomemom & 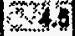 & & & & & & 0.35 & فئ3 & 24 & Notoo & 340 & Fis & 2,200 & 2,720 \\
\hline 1018 & 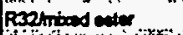 & 7.0 & & & & & 5.0 & 0.22 & $>30$ & $4,070^{\circ}$ & 4,990 & 5 & 17 & 40,900 & 16,700 \\
\hline : & 1 os ondide & 48 & & & 9 & & 6 & 50.13 & 230 & $3,000$. & 6,330 & $x=13$ & 8232 & 13,400 & 10200 \\
\hline 80 & RI2Q ytoneane & 2.5 & & & & & 9.4 & 0.00 & $<0.1$ & 3 & & & 2,470 & & 1,400 \\
\hline bit & Ris & & & & & & tis & 00 & $5: 4$ & 8 & 280 & & 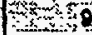 & 9 & 10,800 \\
\hline 22 & R12sprinctod elo & 2.5 & & & & & 5.5 & 0.00 & 10.5 & $\tilde{0}$ & & & 5 & 5.660 & 15,100 \\
\hline 23 & 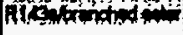 & 28 & & & & 42 & 72 & $\mid$ & 208,3 & 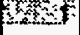 & $x=2 \pi$ & ens & $y \leqslant 210$ & $y=6,100$ & 20,400 \\
\hline & 1000 & & & & & & & & & & & & & & \\
\hline it: & Bition & $x_{2} 0$ & & & & & & 0,0 & 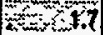 & W & & 70 & 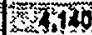 & $y 27$ & 710 \\
\hline-12 & R12min & 3.0 & & & & & 5.2 & 0.00 & 0.1 & & & 62 & 3,060 & & 890 \\
\hline 3. & 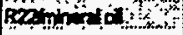 & 35: & & & & & 12 & 0.00 & $\$ 1$ & 60 & $-20,000$ & $3=750$ & $=10.300$ & 16 & 3780 \\
\hline 14 & Rizstipord of & 2.5 & & & & & & 0.26 & 0.9 & 2 & & & 3,850 & & 1,120 \\
\hline$\ldots$ & Bisu & 26 & & & & & & 0.07 & 70 & & 0 & & $=0$ & 101,200 & 119,400 \\
\hline 16 & R134ebrancted eatery & 2.5 & & & & & 5.8 & 0.00 & & 0 & & 0 & 14 & 11,600 & 17,100 \\
\hline A7. & 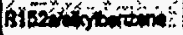 & $x$ & & & 200 & & $x_{1}^{x} 72$ & $: 0.12$ & 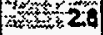 & 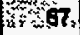 & $10+3320$ & 2000 & $=32$ & $25.410^{\circ}$ & 2.400 \\
\hline 18 & 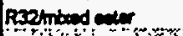 & 6.5 & 1 & & & & 5.2 & 0.21 & 230 & 3,720 & 8,880 & & 0 & 43,600 & 38,900 \\
\hline : & 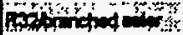 & 39 & & $=0$ & & & 5.0 & 0.28 & 330 & 340 & $=0,250$ & $3 \%$ & $x=23$ & 8,330 & 12,100 \\
\hline-50 & RI24-ybereane & 2.5 & & & & & 8.9 & 0.00 & $<0.1$ & 5 & 28 & & 2,350 & & 1,320 \\
\hline & 10 & & & & & & 8.4 & 0,0 & 8.6 & 4 & $10: 0$ & $\exists=0$ & 25 & $\therefore$ irooio & 10,000 \\
\hline & & & & & & & & 1.27 & & 0 & & & 10 & 6,540 & 16,900 \\
\hline & & & & & & & & 0.00 & 100 & & & & 22 & ino & tizion \\
\hline
\end{tabular}

Table 3-14. Desiccant X: $3 \AA$ Core (No Carbon)

\begin{tabular}{|c|c|c|c|c|c|c|c|c|c|c|c|c|c|c|c|}
\hline Code & Syctem Fucds & $\begin{array}{l}\text { Llowat } \\
\text { color } \\
\text { rest }\end{array}$ & \begin{tabular}{|l|} 
Detie \\
Color \\
p-s
\end{tabular} & 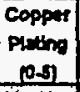 & $\begin{array}{c}\text { solves } \\
\text { formation } \\
\text { pell }\end{array}$ & $\begin{array}{c}\text { otion } \\
\text { corrostion } \\
\text { (a-s) }\end{array}$ & $\begin{array}{c}\text { Crush } \\
\text { strength } \\
\text { (lba) }\end{array}$ & 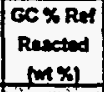 & 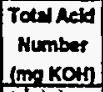 & $\begin{array}{l}F \text { lon in } \\
\text { Luquid } \\
\text { (pponi) }\end{array}$ & 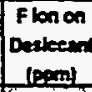 & $\begin{array}{l}\text { Clion in } \\
\text { Liquis } \\
\text { (opm) }\end{array}$ & 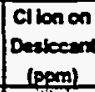 & $\begin{array}{l}\text { Ory Acld } \\
\text { in Llquid } \\
\text { (ppomin) }\end{array}$ & {$\left[\begin{array}{c}\text { Org Act } \\
\text { on Deske } \\
\text { (pem) }\end{array}\right.$} \\
\hline XNETK & 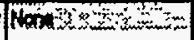 & 23 & & & 8 & -1 & 60 & 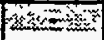 & $x=$ & & sint & $\sin ^{2}$ & 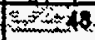 & 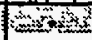 & $3=0$ \\
\hline B11: & Kivanirua & $3: 5$ & & & & & & 000 & $\infty 1$ & & 200 & 300 & $\pi \operatorname{san}$ & 2 & 500 \\
\hline$x-12$ & R12hinord ol & 2.5 & & & & & 7.7 & 0.08 & 0.4 & & 36 & .65 & 2,480 & 0 & $\begin{array}{r}130 \\
130\end{array}$ \\
\hline $1 \times-13$ & 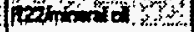 & $3 x$ & & & iv & & 67 & 0.00 & $x<1$ & & 29060 & (n) & 20590 & 5 & 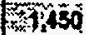 \\
\hline$x-14$ & Ri23minare of & 2.5 & & & & & 4.7 & 0.12 & $<0.1$ & A & & 45 & 1,300 & 0 & 140 \\
\hline 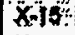 & 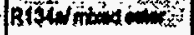 & & & & & & $6: 1$ & 800 & 68.2 & & -20 & $(x+1)$ & Pats & $\therefore 11900$ & 16,600 \\
\hline & Risubrancted ade & 25 & & & & & 7.2 & 0.00 & & & & & 15 & 6,400 & .12300 \\
\hline$x \in t$ & 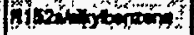 & & & & & & 6. & 13 & 20 & 80 & $=7,70$ & Wn & 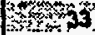 & $14,45:$ & $f=12$ \\
\hline$x-18$ & 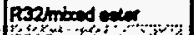 & 7.0 & & & & & & 0.22 & $>30$ & 4780 & 7,090 & & & 48,100 & 22800 \\
\hline Xinto: & 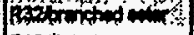 & & & & & & 7,3 & 023 & $x=30$ & 1930 & 81320 & 30 & 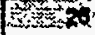 & Eiono & 30,300 \\
\hline$x-20$ & R120 ybonzene & 25 & & & & & 22 & 0.00 & $<0.1$ & & 120 & 160 & 900 & 130 & 1,870 \\
\hline$x-210$ & now & & & & & & (5) & 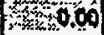 & 81 & & 29 & Sto & 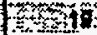 & 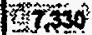 & 16,700 \\
\hline$x-2$ & R12strendred ener & 25 & & & & & 40 & 0.00 & 9.7 & 3 & 0 & 8 & 19 & 4.810 & 11,000 \\
\hline$i x$ & Hilomomed & 2 & & & & & 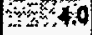 & $=0.00$ & $x_{x \rightarrow 2}=12$ & 22 & $=3 x$ & fents & mast? & 3020 & 13,100 \\
\hline & & & & & & & & & & & & & & & \\
\hline $4 i^{\circ}$ & Bittith & 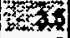 & & & & & 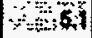 & 000 & 80.6 & & 0100 & 300 & xis?o & 50 & $5=0$ \\
\hline$x-42$ & R12hinuered od & 2,5 & & & & & & 0.00 & $\infty .1$ & & 72 & & 2,360 & & 590 \\
\hline$x=3$. & of & 37 & & & & & & 0.00 & 0.2 & $=120$ & $2,2,40$ & 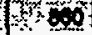 & 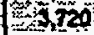 & ress & 2,40 \\
\hline$x-4$ & R123minerd od & 2.5 & & & & & & 0.15 & & & 10 & & 1,340 & & -150. \\
\hline$x>15$ & RISNinbod & 26 & & & & & 7,0 & 00,00 & $=8.7$ & & 20 & Nato & 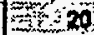 & 9,40 & 130,700 \\
\hline$x-46$ & R134apranctiod ader & 2.5 & & & & & & 0.13 & 11.2 & & & & & 7,200 & 16,000 \\
\hline$: x-i$ & Af & 25 & & & & & 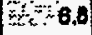 & 0033 & $W=3.1$ & 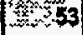 & $=1,050$ & 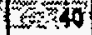 & $x=316$ & 123ato & Kobio: \\
\hline$x-4$ & R32mado & $\begin{array}{r}-7.5 \\
\end{array}$ & & & & & 7.5 & 0.25 & 230 & 4110 & 6,150 & 23 & $\begin{array}{r}39 \\
\end{array}$ & 25,100 & 23,900 \\
\hline & & mote & & & & & 110 & 0.27 & 330 & 4050 & $x=9,170$ & $y=0$ & 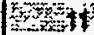 & 516,300 & 20,100 \\
\hline$x-50$ & R124) & 25 & & & & & 6.0 & 0,00 & $<0.1$ & & 36 & 15 & 1060 & & 1,580 \\
\hline$x+6$ & $\operatorname{lm} \min _{n=1}$ & 42 & & & & & & 0.00 & & & 10 & & & 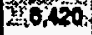 & is. \\
\hline$x=52$ & 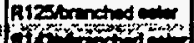 & 2.5 & & & & & 4.0 & 0.00 & 8.8 & 0 & & & 0 & 4,10 & 11,700 \\
\hline & & & & & & & & & & & & & & & \\
\hline
\end{tabular}


Table 3-15. Desiccant Y: $4 \AA$ Core (No Carbon)

\begin{tabular}{|c|c|c|c|c|c|c|c|c|c|c|c|c|c|c|c|}
\hline Code & Ixtm Finde & $\begin{array}{l}\text { Lethe } \\
\text { Colox } \\
\text { (2-1) }\end{array}$ & {$\left[\begin{array}{l}\text { Deats } \\
\text { color } \\
p-3\end{array}\right.$} & $\begin{array}{l}\text { Copper } \\
\text { Ptuting } \\
0-9\end{array}$ & $\begin{array}{c}\text { solds } \\
\text { formition } \\
\text { pes] }\end{array}$ & $\begin{array}{c}\text { stid } \\
\text { corrocton } \\
0 .-1)\end{array}$ & $\begin{array}{c}\text { Crush } \\
\text { strungeth } \\
\text { ploge }\end{array}$ & $\begin{array}{c}G C \times R A f \\
\text { Rasctod } \\
(m \times)\end{array}$ & 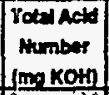 & 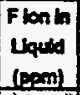 & 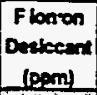 & 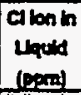 & 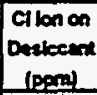 & 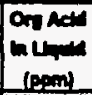 & لren \\
\hline \multirow[t]{2}{*}{ YNAM } & 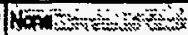 & 828 & & 4 & & & 3.1 & 5 & int & & $3 x+14$ & & 40 & 8 & 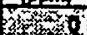 \\
\hline & 16 & & & & & & & & & & & & & & \\
\hline 11 & Rithiners oflot & 20 & & & & & 3.7 & 6.0 & 80.1 & & 2 & क & 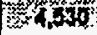 & 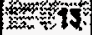 & isto \\
\hline$Y-12$ & R12minered ad & 3.0 & & & & & 4.9 & 0.00 & $\infty .1$ & & 72 & 80 & 5,940 & 3 & 1,010 \\
\hline$Y-13$ & freshinat al on: & 7.0 & & & & & 72.2 & 20.00 & 0.2 & & 5,250 & 810. & 10,600 & nition & 40 \\
\hline$Y-14$ & R123tmmared of & 3.0 & & & & & 5.7 & 0.67 & $<0.1$ & 3 & 13 & 120 & 4,230 & 16 & 250 \\
\hline$Y-15=$ & 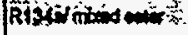 & 2 & & & & & 8 & 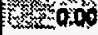 & 50 & & & 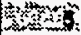 & 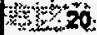 & 1230 & 1820 \\
\hline$Y-16$ & R134abrenched outex & 2.5 & & & & & 7.4 & 0.00 & 5.7 & n & 2 & 5 & 17 & 5,130 & 13,400 \\
\hline 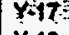 & 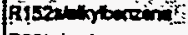 & $5 \%$ & & & & & $\$ 2$ & 0.9 & 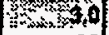 & 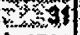 & $\alpha, 430$ & 100 & 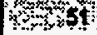 & 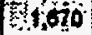 & 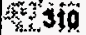 \\
\hline$Y-18$ & R32mbed eter & 4.0 & & & & & 4.7 & 1.16 & $>30$ & 90 & 11,700 & 9 & 24. & 29,400 & 83,300 \\
\hline$Y=18$. & 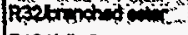 & 30 & & & & & 5.4 & 0.10 & 830 & & 47830 & 17 & 20 & 18,600 & 7,100 \\
\hline$Y-20$ & R124tyberzano & 2.5 & & & & & 4.3 & 0.06 & $\infty .1$ & & 1,110 & 15 & 4,320 & & \\
\hline$|y-z| x$ & 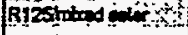 & & & & & 2 & (b) & $6 \%$ & 76 & & & & 17 & 6300 & $20 ; 000$ \\
\hline$Y-22$ & Ri2strenched werer & 2.5 & & & & & 8.0 & 0.00 & 6.3 & 0 & o & 6 & 41 & 5,620 & 19,400 \\
\hline$y=3$ & 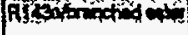 & 20 & & & 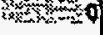 & 28 & (4, 1 & 820000 & 20.1 & $\mid x=12$ & 促 & s. & 100 & 6,490 ? & 77,000 \\
\hline & $1000 p_{p}$ & & & & & & & & & & & & & & \\
\hline$Y+1$ & Bithmoni a & 0 & & & & & 6.0 & 0 & wo.s & & & 240 & 7.90 & $\mid$ & Hrop \\
\hline$Y-12$ & Ri2kinaral od & 3.0 & & & & & 7.6 & 0.00 & $<0.1$ & & 220 & & 8,260 & & 2,240 \\
\hline$Y=3$ & Realminumition & & & & & & 5.7 & 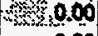 & $\infty .4$ & & 5,680 & $=8070$ & 16,700 & Fit? & $3<30$ \\
\hline$Y+4$ & Rizzmand od & 3.0 & & & & & 5.7 & 0.60 & 0.4 & & 18 & 54 & 840 & 6 & 290 \\
\hline$x+25$ & 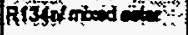 & & & & & & & 0.00 & 10,0 & & & Wing & 38 & 7,000 & $17 \%, 200^{\circ}$ \\
\hline$y+46$ & 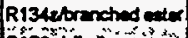 & 2.5 & & & & & & 0.00 & 13.0 & & 0 & & 71 & 4,640 & 19,800 \\
\hline$Y-47$ & 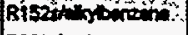 & 4 & & & & & 73 & $=028$ & 22 & 10 & 430 & 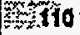 & $x=4 f$ & Fition & $160^{\circ}$ \\
\hline$Y+48$ & Rzentudadir & 4.0 & & & & & & 0.24 & $>30$ & 3340 & 8,010 & & & 40,000 & 20,900 \\
\hline$\gamma+8^{\circ}$ & 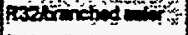 & 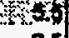 & & & & & 40 & 50.32 & 330 & 31350 & 10,100 & & $3 x+36$ & 16,800 & 17,2000 \\
\hline$Y-50$ & 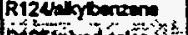 & 2.3 & & & & & 7.2 & 0.00 & $<0.1$ & & 250 & 59 & 2,710 & 9 & 1,910 \\
\hline 5 & & & & & & & 5.3 & $0: 36$ & 6.5 & & & & & 8,600 & 28,300 . \\
\hline$Y-52$ & thed extar & 2.5 & & & & & 4.9 & 0.00 & 13.7 & & & & 27 & 8,100 & 19,000 \\
\hline & & & & & & & & 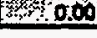 & 28.96 & & & & $=210$ & 1000 & 20.100 \\
\hline
\end{tabular}

\section{Table 3-16. Desiccant X: $4 \AA$ Core (No Carbon)}

\begin{tabular}{|c|c|c|c|c|c|c|c|c|c|c|c|c|c|c|c|}
\hline Code & system fluids & $\begin{array}{l}\text { Lquidd } \\
\text { color } \\
1241\end{array}$ & $\begin{array}{l}\text { Deste } \\
\text { color } \\
\text { ous }\end{array}$ & $\begin{array}{c}\text { Copper } \\
\text { Pesting } \\
\text { pas }\end{array}$ & $\begin{array}{c}\text { Sollds } \\
\text { formation } \\
\text { (0) }\end{array}$ & $\begin{array}{c}\text { Sied } \\
\text { corroston } \\
\text { (0) } \\
\end{array}$ & \begin{tabular}{|c|}
$\begin{array}{c}\text { Cruseh } \\
\text { strength } \\
\text { (1bes) }\end{array}$ \\
\end{tabular} & $\begin{array}{c}G C * R \text { RI } \\
\text { Rexted } \\
(m x)\end{array}$ & $\begin{array}{l}\text { Tetw actd } \\
\text { number } \\
\text { Inge KOHI) }\end{array}$ & $\begin{array}{l}\text { Flonn } \\
\text { Lquida } \\
\text { (ppomit }\end{array}$ & $\begin{array}{c}\text { Flon on } \\
\text { Desticemtent } \\
\text { (pom) }\end{array}$ & $\begin{array}{l}\text { C1tonth } \\
\text { uquid } \\
\text { (pom) }\end{array}$ & $\begin{array}{c}\text { Cikonon } \\
\text { Deskeant } \\
\text { (ppond) }\end{array}$ & $\begin{array}{l}\text { Org nedd } \\
\text { in Liquid } \\
\text { (ppem) }\end{array}$ & $\begin{array}{c}\text { org hatd } \\
\text { on Dariks } \\
\text { (ppem) }\end{array}$ \\
\hline 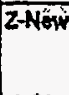 & $\begin{array}{l}\text { Hopin } \\
50 \mathrm{ppm} \text { Houture }\end{array}$ & $=28$ & & & 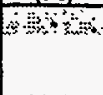 & 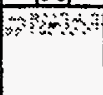 & 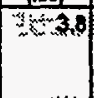 & 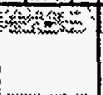 & 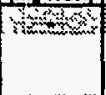 & 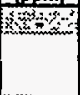 & 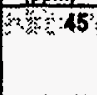 & $x_{0}=i^{\circ}$ & 3954 & $x=6$ & $x, y=0$ \\
\hline$-11 f^{2}$ & Rivin & 30 & & & & & $\times 2,3$ & 0,15 & 然it & 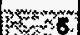 & 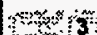 & & 6076 & $y=34$ & 20" \\
\hline$z-12$ & R!2 meneral al & 3.0 & & & & & 2.8 & 0.00 & $<0.1$ & 4 & 280 & 34 & $.3,190$ & 0 & 1,120 \\
\hline $2-13$ & 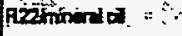 & 29 & & & & & $2 i$ & o.11 & $<0 . \mathrm{i}$ & 1853 & 3,680 & 620 & 16,900 & 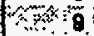 & 3,620 \\
\hline $2-14$ & Ri23trineral of & 3.0 & & & & & 3.4 & 0.25 & 0.3 & & 280 & 64 & 3,050 & 15 & 360 \\
\hline$z=15$ & 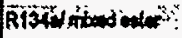 & 20 & & & & & 3.5 & 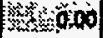 & X & 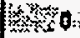 & i. & & 74 & 6060 & 18,400 \\
\hline Z-16 & netrod aterex. & 2.5 & & & & & 3.3 & 0.00 & & & & & & 5,010 & 24,500 \\
\hline $2-17$ & 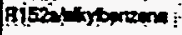 & 500 & & & & & misol & 0.43 & 20 & कere & 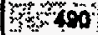 & 2020 & m.6.6. & 2400 & 404 \\
\hline Z-18 & Rasminod enter & 6.0 & & & & & 1.7 & 0.00 & $>30$ & 4.330 & 15,500 & 16 & 18 & 32,200 & 30,400 \\
\hline $2-1 \theta^{\circ}$ & Ristionatiod & 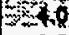 & & & & 2 & 54 & 1.17 & $3 \times 30$ & $8 ;, 600$ & 16,400 & $\approx 6$ & $x_{0}=x+y$ & 20,000 & 10800 \\
\hline $2-20$ & 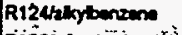 & 2.5 & & & & & 4.2 & 0.49 & $<0.1$ & 14 & 1,290 & & 3,290 & & 410 \\
\hline $2,2 t^{5}$ & 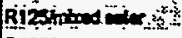 & 3 & & & & & 4⿻ & (0.00 & ?77? & 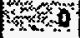 & $m$ & 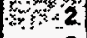 & thons & 0,0409 & 21,000 \\
\hline $2-22$ & 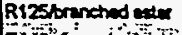 & 2.5 & & & & & 4.8 & 0.00 & 13.9 & 0 & & & 13 & 8,870 & 16,300 \\
\hline $2-23:$ & 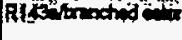 & 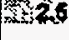 & & & & & 3.4 & 0.00 & $8=7$ & $x$ & m: 300 & $x=6$ & 30 & 500 & 17,100 \\
\hline & 1000 & & & & & & & & & & & & & & \\
\hline $2-44$ & Rithen & 30 & & & & & 4.4 & 0.00 & 40 & 10 & $=200$ & 140 & 5,900 & $\approx 0$ & 40 \\
\hline $2-42$ & Ri2minerd of & 3.0 & & & & & 3.9 & 0.00 & $<0.1$ & 1 & & & 3,850 & & 1,350 \\
\hline $2-43$ & Rónhheral of & 2 & & & & & 3.9 & 0.00 & (0.1) & 25 & $=-3,080$ & 743 & 14200 & 4 & 2,0090 \\
\hline $2+4$ & R123minered od & 3.0 & & & & & 2.8 & 0.28 & $<0.1$ & 5 & 420 & 53 & 3,250 & & 800 \\
\hline $2+3$ & 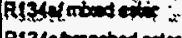 & 27 & & & & & 3.8 & 0.000 & 8.0 & 0 & 1 & 10 & 62 & 0,650 & 20.600 \\
\hline $\begin{array}{l}z-46 \\
z+7\end{array}$ & 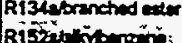 & 25 & & & & & 8.3 & 0.00 & 11.6 & 0 & 0 & & 83 & 5,460 & 25,100 \\
\hline 248 & 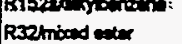 & $-\infty$ & & & & & 4.0 & $\begin{array}{l}0.43 \\
0.22\end{array}$ & $>3.2$ & $\begin{array}{l}6000 \\
5,30\end{array}$ & $=830$ & $\begin{array}{r}70 \\
45\end{array}$ & 36 & $\because 70$ & 286 \\
\hline & 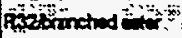 & & & & & & 3.0 & 0.17 & 830 & 10,000 & $\because 1,610^{\circ}$ & & $\begin{array}{l}27 \\
38\end{array}$ & $\left|\begin{array}{r}45,400 \\
15,800\end{array}\right|$ & $\begin{array}{r}34,900 \\
19,300\end{array}$ \\
\hline 2 & Ri24: & 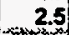 & & & & & 9.8 & 0.00 & $<0.1$ & 3 & & 24 & 220 & 1,370 & 33,200 \\
\hline & RARSthat & $x+m$ & & & & & 4.7 & -0.00 & सt & $\because 0$ & $\% 0$ & & $x, 28$ & $8,330^{\circ}$ & 28,300 \\
\hline 52 & & 2.5 & & & & & $\begin{array}{r}3.7 \\
3.3 .1\end{array}$ & 0.00 & 9.6 & 每 & & 6 & 8 & 4,440 & 14,900 \\
\hline & & & & & & & & & & & & & 14.1. & 4.0800 & 23 \\
\hline
\end{tabular}




\section{COMPATIBILITY OF MANUFACTURING PROCESS FLUIDS WITH HIC REFRIGERANTS AND ESTER LUBRICANTS}

\section{Objective:}

To provide information that will enable manufacturers of components of air-conditioning and refrigeration equipment to select reliable process fluids.

\section{Results:}

Imagination Resources, Inc., is performing this research under contract to ARTI.

Part I of this project was a survey of manufacturers and fluid suppliers to determine what processing fluids are used by the industry and what testing has been performed previously on these compounds. The survey has been completed, major component manufacturers submitted lists of fluids to be analyzed. The list of fluids obtained in the survey was narrowed down to a workable size for further experimental testing. Fluids covered included soldering fluxes, cleaning fluids, lubricating fiuids, rust inhibitors agents and adhesives. Interim report, DOE/CE/23810-43, Compatibility of Manufacturing Process Fluids with HFC Refrigerants and Ester Lubricants, by Richard C. Cavestri, Ph.D., dated November 1994; summarizes the findings of the survey and outlines the experimental method for Part II of the project.

\section{Test Fluids Included}

\section{3 bronzing fluxes}

8 coolants

15 detergents, degreasers, or cleaners

4 iron phosphatizers

13 lubricants

17 rust inhibitors or preventatives

4 sealants

Part II of the project consists of experimental measurements of the compatibility of 64 fluids which were selected in Part I. It included'sealed tube stability and miscibility testing. Results of these tests will determine the chemical and thermal stability of the fluids and also their solubility characteristics in HFC refrigerants. The critical solution temperature were measured for several different concentrations of each processing fluid in HFC-134a and two different ester lubricants. The final report is being drafted. 
The contractor has developed an analytical method using size exclusion chromatography which will allow him to quantitatively detect the amount of each processing fluid in the refrigerant/lubricant solution, even when the fluid appears to be immiscible. Those process fluids which are found to have suitable miscibility characteristics for use in HFC systems will be further tested for chemical and thermal stability using standard sealed tube test methods. 
COMPATIBILITY OF MOTOR MATERIALS

USED IN AIR-CONDITIONING FOR RETROFITS

WITH ALTERNATIVE REFRIGERANTS AND LUBRICANTS

\section{Objective:}

To examine the compatibility of motor materials for retrofit with alternative refrigerants and lubricants.

\section{Results:}

The Trane Company is conducting this research under contract to ARTI. A report of the results to date are detailed in the quarterly technical report, DOE/CE/23810-51B, Compatibility of Refrigerants and Lubricants with Motor Materials Under Retrofit Conditions, by Robert Doerr and Todd Waite, January 1995. The results are summarized below.

The project is investigating the material compatibility of motor materials under the following retrofit scenarios:

CFC-11/mineral oil to HCFC-123/mineral oil CFC-12/mineral oil to HFC-134a/polyolester lubricant R-502/mineral oil to R-404A/polyolester (HFC-125/HFC-143a/HFC-134a) HCFC-22/mineral oil to R-407C/polyolester (HFC-32/HFC-125/HFC-134a) CFC-11/mineral oil to HFC-245ca/polyolester .

HCFC-123/mineral oil to HFC-245ca/polyolester

Compatibility test of motor materials with the first four retrofit scenarios have been completed. The tests were conducted in accordance with UL Standard 2171. Test specimens of motor materials and motorettes were sequentially exposed for 500 hours in the initial refrigerant-lubricant mixture, then exposed to the alternative refrigerantlubricant mixture and evaluated after 168, 336 and 500 hours. Exposures involving CFC$12, \mathrm{R}-502$ and $\mathrm{HCFC}-22$ retrofit scenarios were exposed at a temperature of $127^{\circ} \mathrm{C}$ $\left(260^{\circ} \mathrm{F}\right)$. Exposures for the CFC-11 retrofit scenario were exposed at a temperature of $100^{\circ} \mathrm{C}\left(212^{\circ} \mathrm{F}\right)$. Motor materials tested were:

\section{Magnet Wire Insulation}

- polyester base with amide imide overcoat

- $\quad$ esterimide base with amide imide overcoat

- polyester base with amide imide overcoat ${ }^{1}$ and epoxy saturated glass serving 


\section{Varnishes}

- water base epoxy-phenolic: Isopoxy 800

- $\quad$ solvent-epoxy-phenolic: P.D. George 923

- solvent-epoxy: Sterling U-475EH ${ }^{1}$

\section{Sheet Insulation}

- polyester film: Mylar

- polyester film, low oligomer: Melinex

- polyester composite: Darcon-Mylar-Darcon

- aramid fiber mat: Nomex $41010 \mathrm{mil}$

- aramid fiber mica mat: Nomex Mica

- aramid mat, polyester film composite: Nomex-Mylar-Nomex

\section{Spiral Wrapped Sleeving}

- polyester film

- $\quad$ aramid fiber mat, polyester film

Lead Wire Insulation

- polyester composite: Darcon-Mylar-Darcon

- polyester, fluoropolymer composite: Darcon-Teflon-Darcon

\section{Tie Cords}

- polyester

\section{Assembly Tapes}

- $\quad$ braided polyester, acrylic binder

- polyester mats

'Only tested with CFC-11/mineral oil and HCFC-123/mineral oil.

The above motor materials appear to be compatible with the alternative refrigerant/lubricant mixture for the retrofit scenarios tested. The only concerns were embrittlement of the polyethylene phthalate (PET) which is found in Mylar and Melinex sheet and sleeving insulation, and delamination and blistering of the Nomex composite sheet insulation in HCFC-22, R-502 and CFC-12 and separation of the 475 varnish from metal surfaces in HCFC-123. The sheet and sleeving insulation were affected by the old refrigerant/mineral oil and further degraded in the alternative refrigerant/lubricant mixture. The separation of the 475 varnish from the metal surfaces may have been influenced by . the condition of the metal surface before application of the varnish. 


\section{PRODUCTS OF MOTOR BURNOUTS}

\section{Objectives:}

To identify and quantify the products of motor burnouts in systems with R-22 with mineral oil, R-134a with polyolester lubricant, and R-50 $7^{1}$ with polyolester lubricant. .

To correlate the toxic nature of the identified products of motor burnouts on humans, from existing literature.

- To assess the corrosive effects of these products on the electric feed-through terminals.

- To assess the efficacy of currently used procedures which use filter dryers to remove the residual burnout products and prevent repeat burnouts.

- To assess whether HFC refrigerant/lubricant systems are likely to increase or decrease the incidence of motor burnouts as compared to HCFC/lubricant systems.

\section{Results:}

The Lawrence Livermore National Laboratory (LLNL) is conducting this research under contract to ARTI. A summary of its progress is contained in the quarterly technical report, DOE/CE/23810-51D, Products of Motor Burnouts, by Ruth Hawley-Fedder, Ph.D., April 1995.

An initial literature search of available data has been completed. LLNL has completed electrical breakdown testing of R-22; R-134a and R-507 at atmospheric pressure and ambient temperature. Results are presented in Tables 6-1 through 6-3.

LLNL has also designed and in the process of constructing a test stand for testing at temperatures up to $200^{\circ} \mathrm{C}$ and pressures up to $3450 \mathrm{kPa}$ (500 psi).

\footnotetext{
${ }^{3} \mathrm{R}-507$ is a blend consisting of HFC-125 and HFC-143a at a 50/50\% composition by weight.
}

$$
6-1
$$


Table 6-1. R-22 Breakdown Products - Atmospheric Pressure

\begin{tabular}{|c|c|c|c|c|c|c|c|}
\hline & & \multicolumn{6}{|c|}{ Amount Formed (normalizad to R-22) } \\
\hline & Tont ID & $\begin{array}{l}950111 \mathrm{~b} \\
100 \mathrm{~K} * 2 \\
\end{array}$ & $\begin{array}{l}950109 a \\
100 \mathrm{~K}\end{array}$ & $\begin{array}{l}950105 f \\
50 K \quad 11 \\
\end{array}$ & $\begin{array}{r}950110 \mathrm{C} \\
50 \mathrm{~K} \quad 2\end{array}$ & $\begin{array}{r}950509 \mathrm{~b} \\
10 \mathrm{~K}=2 \\
\end{array}$ & $\begin{array}{l}950111 b \\
10 K \quad 13 \\
\end{array}$ \\
\hline & energy (joules) & 262 & 262 & 131 & 131 & 26.2 & 26.2 \\
\hline $\begin{array}{c}\text { RT } \\
(\min )\end{array}$ & Tentative Compound ID & & & & & & \\
\hline 7 & Tetrafluoroethene & 26.49 & 24.94 & 12.80 & -14.441 & $3.31:$ & 2.85 \\
\hline 8 & Hexafluoropropene & 4.32 & 3.90 & 1.29 & 1.49 & $0.28 !$ & 0.14 \\
\hline 11 & Chlorodifluoromothane (R22) & 100 & 100 & 100 & 100 & $100 !$ & 100 \\
\hline 13 & dichlorodifluoromethane (R12) & 16.93 & 14.65 & 6.14 & 7.20 & $1.36 !$ & 0.80 \\
\hline 15 & 1-chloro-1,1,2,2,-tetrafluoroethane & 0.49 & 0.35 & 0.13 & 0.15 & $0.00 !$ & 0.00 \\
\hline 17 & 2-chloro-1,1,3,3,3 -pentafluoro-1-propene & 0.47 & 0.34 & 0.13 & 0.14 & 0.001 & 0.00 \\
\hline 171 & 3-chloro-1,1,2,3,3-pentafluoro-1-propene & 0.93 & 0.66 & 0.25 & 0.27 & 0.00 & 0.00 \\
\hline 181 & 1,2-dichloro-1,1,2,2-tetrafluoroethane & 5.79 & 4.92 & 1.77 & 1.99 & 0.35 & 0.17 \\
\hline 19 & $\mathrm{CrCCF}_{3}$ & 0.87 & 0.65 & 0.24 & 0.26 & 0.00 & 0.04 \\
\hline 21 & chlorohexafluoropropane & 0.17 & 0.11 & 0.05 & 0.10 & 0.00 & 0.00 \\
\hline 22 & chlorodifluoromethane & 0.49 & 0.43 & 0.08 & 0.07 & 0.00 & 0.00 \\
\hline 25 & $\mathrm{CF}_{2} \mathrm{CACF}_{2} \mathrm{CF}_{2} \mathrm{O}$ & 0.34 & 0.03 & 0.11 . & 0.11 & 0.00 & 0.00 \\
\hline 25) & $\mathrm{CF}_{3} \mathrm{CFCF}_{2} \mathrm{a}$ & 1.28 & 1.07 & 0.38 & 0.42 & 0.07 & 0.03 \\
\hline 26 & 1,3-Butadiyne & 0.00 & 0.08 & 0.00 & 0.00 & 0.00 & 0.00 \\
\hline 27 & dichlorofluoromethane & 0.10 & 0.09 & 0.00 & 0.00 & 0.00 & 0.00 \\
\hline $27 \mid$ & 1,1-dichloro-2,2-difluoroethene & 1.50 & 1.22 & 0.46 & 0.52 & 0.10 & 0.08 \\
\hline 28 & 1,2-dichloro-1,2-difluoroethene & 0.76 & 0.63 & 0.22 & 0.30 & 0.04 & 0.03 \\
\hline 28 & 1,2-dichloro-1,2-difluoroethene & 0.91 & 0.75 & 0.24 & 0.33 & 0.04 & 0.06 \\
\hline 301 & trichlorofluoromethane & 0.66 & 0.51 & 0.16 & 0.24 & 0.041 & 0.00 \\
\hline 31 & Chloropentafluoroethane & 0.80 & D.56 & 0.23 & 0.26 & 0.04 & 0.03 \\
\hline 31. & 1,2-dichloro-1,3,3,3-tetrafluoro-1-propene & 0.20 & 0.18 & 0.07 & 0.09 & 0.00 & 0.00 \\
\hline 32 & 1,2-dichloro-1,3,3,3-tetrafluoro-1-propene & 0.42 & 0.27 & 0.14 & 0.15 & 0.04 & 0.00 \\
\hline 32 & dichloroethyne & 0.69 & 0.57 & 0.20 & 0.24 & 0.05 & 0.04 \\
\hline $33 \mid$ & C4F3Cl & 0.19 & 0.17 & 0.08 & 0.06 & 0.00 & 0.00 \\
\hline 341 & 1,1,2-trichloro-1,2,2-trifluoroethane & 0.29 & 0.26 & 0.11 & 0.10 & 0.06 & 0.00 \\
\hline 351 & Imonochloro??? & 0.80 & 0.73 & 0.26 & 0.25 & 0.07 & 0.00 \\
\hline $35 \mid$ & ?????? & 0.22 & 0.16 & 0.10 & 0.06 & 0.00 & 0.00 \\
\hline 36 & chloropentafluoroethane & 0.47 & 0.39 & 0.15 & 0.17 & 0.05 & 0.00 \\
\hline 36 & Itrichloropropene & 0.43 & 0.18 & 0.06 & 0.00 & 0.00 & 0.00 \\
\hline 37 & 1.2-dichlorotetrafluorocyclobutene & 0.46 & 0.44 & 0.16 & 0.19 & 0.06 & 0.00 \\
\hline 37 & 1,2-dichlorotetrafluorocyclobutene & 0.47 & 0.26 & 0.04 & 0.00 & 0.00 & 0.00 \\
\hline 38 & 1,2-dichlorotetrafluorocyclobutene & 0.17 & 0.14 & 0.06 & 0.05 & 0.00 & 0.00 \\
\hline 381 & ???? & 0.72 & 0.13 & 0.08 & 0.05 & 0.00 & 0.00 \\
\hline 38 & $? ? ?$ & 0.61 & 0.14 & 0.06 & 0.05 & 0.00 & 0.00 \\
\hline 38 & 1,2,2-trichloro-1,1,3,3,3-pentafluoropropane & 0.96 & 0.84 & 0.40 & 0.21 & 0.08 & 0.00 \\
\hline 39 & chloropentafluoroethane & 0.35 & 0.32 & 0.11 & 0.11 & 0.00 & 0.00 \\
\hline 41 & trichlorofluoroethylene & 0.68 & 0.60 & 0.18 & 0.24 & 0.05 & 0.03 \\
\hline 43 & $? ? ?$ & 0.54 & 0.13 & 0.08 & 0.00 & 0.00 & 0.00 \\
\hline 43 & unknown & 0.16 & 0.18 & 0.06 & 0.07 & 0.00 & 0.00 \\
\hline 45 & ???? & 0.34 & 0.10 & 0.04 & 0.00 & 0.00 & 0.00 \\
\hline 48 & chloropentafluorobenzene & 0.24 & 0.14 & 0.09 & 0.06 & 0.00 & 0.00 \\
\hline 51 & tetrachlorethene & 0.41 & 0.30 & 0.26 & 0.13 & 0.06 & 0.00 \\
\hline 52 & 1,1,3,3-tetrachloro-2,3-difluoropropene & 0.22 & 0.11 & 0.00 & 0.06 & 0.00 & 0.00 \\
\hline & & & & & & & \\
\hline & TOTAL & 173.33 & 162.58 & 127.45 & 130.60 & 106.15 & 104.30 \\
\hline & Total less R-22 & 73.33 & 62.58 & 27.45 & 30.60 & 6.15 & 4.30 \\
\hline & & & & & & & \\
\hline note: & Amounts are normalized to R-22 as 100 & & & & & & \\
\hline & 1,1,2-trichloro-1,2,2,-trifluoroethane and & & ans & & & & \\
\hline & the presence of these compounds may be & conta & 0 & & & & \\
\hline
\end{tabular}

$$
6-2
$$


Table 6-2. R-134a Brealkdown Products - Atmospheric Prẹssure

\begin{tabular}{|c|c|c|c|c|c|c|}
\hline$\cdot 1$ & Tost ID & $\begin{array}{l}950117 \mathrm{C} \\
100 \mathrm{~K} * 1\end{array}$ & $\begin{array}{l}950118 \mathrm{c} \\
100 \mathrm{~K}: 2\end{array}$ & $\begin{array}{l}950119 b \\
50 K \quad 42\end{array}$ & $\begin{array}{l}\text { I9501180 } \\
\text { I50K \#1 }\end{array}$ & $\begin{array}{l}95001196 \\
10 K+1\end{array}$ \\
\hline & energy (joules) & 256 & .256 & 128 & 128 & 25.6 \\
\hline $\begin{array}{c}\text { RT } \\
(\mathbf{m i n})\end{array}$ & Tentatlve Compound ID $\quad \cdot \cdots$ & & - & & & - \\
\hline 6.98 & Hexafluoroethane & 3.44 & 2.47 & 1.30 & 1.85 & 0.76 \\
\hline 7.23 & Tetrafluoroethene & .10 .49 & 7.17 & 2.33 & 3.77 & 0.46 \\
\hline 7.85 & $1,1,2,3,3,3$-hexafluoro-1-propene & 0.00 & 0.00 & 0.00 & 0.29 & 0.00 \\
\hline 8.02 & Trifluoroethene & 3.05 & 2.35 & 0.60 & 1.03 & 0.00 \\
\hline 8.78 & $1,1,1,2$-tetrafluoroethane $(R-134 a)$ & 100 & 100 & 100 & 100 & 100 \\
\hline 9.97 & unknown & 0.60 & 0.42 & 0.00 & 0.32 & 0.25 \\
\hline 11.12 & chlorodifluoromethane & 1.91 & 0.01 & 0.03 & 0.00 & 0.00 \\
\hline 17.16 & $1,2,3,4,5,5$-hexafluoro-1,3-cyclopentadiene & 0.06 & 0.08 & 0.05 & 0.00 & 0.00 \\
\hline 17.27 & $1,2,3,4,5,5$-hexafluoro-1,3-cyclopentadiene & 0.06 & 0.06 & 0.00 & 0.03 & 0.00 \\
\hline 19.51 & $1,1,1,6,6,6$-hexafluoro-2,4-diyne & 0.08 & 0.11 & 0.03 & 0.02 & 0.00 \\
\hline 20.37 & unknown & 0.02 & 0.03 & 0.00 & 0.00 & 0.00 \\
\hline 21.22 & Octafluoro-1,3,5-Hexatriene & 0.06 & 0.03 & 0.00 & 0.00 & 0.00 \\
\hline 21.44 & unknown & 0.02 & 0.00 & 0.00 & 0.00 & 0.00 \\
\hline 25.87 & C5F5H & 0.04 & 0.04 & 0.01 & 0.03 & 0.00 \\
\hline 26.96 & unknown & 0.04 & 0.04 & 0.00 & 0.03 & 0.00 \\
\hline 35.50 & unknown & 0.02 & 0.03 & 0.00 & 0.00 & 0.00 \\
\hline \multirow[t]{4}{*}{38.72} & unknown & 0.04 & 0.02 & 0.00 & 0.02 & 0.00 \\
\hline & & & & & & \\
\hline & TOTAL & 119.92 & 112.85 & 104.36 & 107.40 & 101.47 \\
\hline & TOTAL less R-134a & 19.92 & 12.85 & 4.36 & 7.40 & 1.47 \\
\hline \multirow[t]{3}{*}{ Note: } & Amounts are normalized to $R-134 a$ as 100 & & & & & \\
\hline & \multicolumn{4}{|c|}{ chlorodifluoromethane is used for instrument calibration; the presence of this } & & \\
\hline & compound may be due to contamination & & & & & \\
\hline
\end{tabular}


Table 6-3. R-507 Breakdown Products - Atmospheric Pressure

\begin{tabular}{|c|c|c|c|c|c|c|}
\hline & & & & & & 1 \\
\hline & & \multicolumn{5}{|c|}{ Amount Formed (normalized to R-507) } \\
\hline & Test to & $\begin{array}{l}950111 \mathrm{~d} \\
100 \mathrm{~K} 1 \mathrm{I1}\end{array}$ & $\begin{array}{l}950123 c \\
100 K: 2\end{array}$ & $\begin{array}{l}950124 \mathrm{C} \\
\text { soK } 22\end{array}$ & $\mid \begin{array}{l}950124 b \\
50 K \approx 1\end{array}$ & $\begin{array}{l}950124 d \\
110 K: 1\end{array}$ \\
\hline & energy (loules) & 2.237 & .237 & -118.5 & 118.5 & 23.7 \\
\hline RT (min) & Tentative Compound ID & & & & & \\
\hline 7.081 & Hexafluoroethane & 2.33 & 2.92 & 1.20 & 9.831 & 0.69 \\
\hline 7.35 & Tetrafluoroethene & 6.55 & 7.07 & 2.88 & 2.48 & 0.68 \\
\hline 7.601 & 1.1-difluoroethene & 3.20 & 3.59 & 1.43 & 0.00 & 0.00 \\
\hline 7.85 & R-507 & 100 & 100 & 100 & 100 & 100 \\
\hline 8.901 & $1,1,1,2$-tetrafluoroethane (R-134a) & 0.48 & 0.22 & 0.19 & 0.04 & 0.20 \\
\hline 9.21 & 1,1,3,3,3-pentafluoro-1-propene & 0.49 & 0.48 & 0.19 & 0.06 & 0.00 \\
\hline 9.72 & 3,3,3-Trifluoro-1-propyne & 0.15 & 0.15 & 0.07 & 0.00 & 0.02 \\
\hline $10.08 \mid$ & hexafluoro-gyclobutene & 0.04 & 0.00 & 0.00 & 0.00 & 0.00 \\
\hline 10.43 & 1.1,3,3,3-hexafluoropropane & 0.06 & 0.00 & 0.00 & 0.001 & 0.00 \\
\hline 11.20 & Chlorodifiuoromethene & 0.03 & 0.03 & 0.01 & 0.00 & 0.03 \\
\hline $12.45 \mid$ & junknown & 0.03 & 0.01 & 0.01 & 0.00 & 0.02 \\
\hline 17.01 & $1,2,3,4,5,5$-hexafluoro-1,3-cyclopentadiene & 0.05 & 0.04 & 0.01 & 0.00 & 0.00 \\
\hline 17.23 & $1,2,3,4,5,5$-hexafluoro-1,3-cyclopentadiene & 0.05 & 0.02 & 0.02 & 0.001 & 0.00 \\
\hline 19.391 & $1,1,1,6,6,6$-hexalluoro-2,4-diyne & 0.10 & 0.07 & 0.01 & 0.00 & 0.00 \\
\hline 20.97 & $\begin{array}{l}4 \text { - (difluoromethylene)-2,3,3- } \\
\text { trifluorocyclobutene }\end{array}$ & 0.01 & 0.03 & 0.00 & 0.00 & 0.00 \\
\hline 21.35 & unknown & 0.01 & 0.00 & 0.00 & 0.00 & 0.00 \\
\hline 25.801 & CSFSH & 0.02 & 0.02 & 0.00 & 0.00 & 0.00 \\
\hline 26.92 & $? ? ? ? ?$ & 0.04 & 0.03 & 0.01 & 0.00 & 0.00 \\
\hline 35.44 & $77 ? ? ?$ & 0.02 & 0.00 & 0.00 & 0.00 & 0.00 \\
\hline 38.68 & ????? & .0 .03 & 0.08 & 0.00 & 0.00 & 0.00 \\
\hline & & & & & & \\
\hline & TOTAL & 113.150 & 114.74 & 106.03 & 112.42 & 101.64 \\
\hline & TOTAL less AZ-50 & 13.139 & 14.74 & 6.03 & 12.42 & 1.64 \\
\hline Note: & Amounts are normalized to R-507 as 100 & & & & & \\
\hline & chlorodifluoromethane is used for instrument cal & libration: the & presence of & & & \\
\hline & this compound may be due to contamination & & & & & \\
\hline
\end{tabular}




\section{ACCELERATED TEST METHODS}

FOR PREDICTING THE LIFE OF MOTOR MATERIALS

EXPOSED TO REFRIGERANT-LUBRICANT MIXTURES

\section{Objectives:}

To develop test methods and procedures to predict the life of motor insulating materials and varnishes used in hermetic motors.

To validate proposed test methods and procedures.

\section{Results:}

The Radian Corporation has completed Phase 1 of this research under contract with ARTI. This phase included a literature search and analysis of current test methods, along with the conceptual design for an improved accelerated test method. Results of this study are presented in the report, DOE/CE/23810-21, Accelerated Test Methods for Predicting the Life of Motor Materials Exposed to Refrigerant/Lubricant Mixtures, Phase 1: Conceptual Design, by Peter F. Ellis II and Alan Ferguson, 11 June 1993 (RDB \#3A17, 68 pages).

As a result of their studies, researchers at Radian found that the majority of hermetic motor insulation failures occur in the stator windings of the motor due to a combination of thermal, chemical, and mechanical interactions. A review of an insurance industry survey [Stouppe and Lau, 1989] indicated that $84.0 \%$. of hermetic motor failures were attributed to stator winding failures.

Radian examined several degradation models and investigated the advantages and disadvantages of the following test methods which are used by industry for testing of hermetic motors:

- motorette test (IEEE Standard 117 \& UL Standard 984-1989),

- sealed tube aging test, and

- plug-reversal test.

The motorette test uses a simplified simulation of stator windings as the test device. The motorette is stressed with electrical potential, but no current, while exposed to a refrigerant-lubricant mixture in a heated autoclave. The motorette test method provides information on the chemical and thermal degradation of insulation materials. However, it does not provide information of degradation due to the differential thermal expansion or magnetic forces on the windings. 
The sealed-tube test developed by General Electric [Spauschus and Sellers, 1969; Spauschus and Field, 1979] used bifilar coils of magnet wire sealed in glass tubes with the refrigerant-lubricant mixtures. Leads of each bifilar coil were sealed through the top of the glass tube, which allowed monitoring of the dielectric properties of the insulation. Although the method was useful for determining the Arrhenius constants of magnet wire varnish insulation degradation, it does not address the degradation of other insulation components and only simulates the thermochemical aging process.

The plug-reversal test uses a hermetic motor-compressor unit as the test device, modifying the compressor so that it can rotate in either direction with equal ease. The unit is placed inside a refrigerant loop. The polarities of two of the three phase wires of the motor are repeatedly reversed, causing the motor to stall and reverse direction with each reversal. Each plug reversal simulates a locked rotor. This test simulates the full range of forces on hermetic motors. However, the overall test apparatus is complex and has two drawbacks. Components of the supporting refrigeration test loop often fail prior to an actual motor failure and purging the entire test loop for subsequent refrigerant-lubricant mixture tests is difficult and costly.

A test method has been proposed that combines the advantages of these test methods into a single practical method. This proposed method uses a stator simulator unit (SSU). The SSU (see Figure 7-1) consists of a laminated electric steel core, simulating the stator stack of a hermetic motor. The core will contain slot insulation, two coils separated by phaseto-phase insulation and slot wedge insulation. The test method exposes the SSU to a refrigerant-lubricant mixture in an autoclave equipped with a headspace chiller and syphon cup similar to those used for motorette tests. Plug-reversal in-rush currents are simulated by intermittent $30 \mathrm{Amp} \mathrm{AC}$ pulses applied to the lead wires of the SSU.

The SSU and test protocol would emulated the following forces which act on motor stator windings and cause insulation failure:

- thermal aging

- chemical aging

- differential thermal expansion

- magnetodynamic forces

- transient voltage stresses from simulated starting cycles.

Several parameters will be used to evaluate SSU performance:

- winding capacitance

- capacitance (power) dissipation factor

- surge testing

- DC high potential testing

- polarization index. 
Industry accepted guidelines exist for evaluating each of these parameters which permit determination of logical test endpoints, before actually reaching a SSU burnout. It is postulated that trend analysis results for each of these parameters may allow projection of the time to a set endpoint well before that end-point is reached. That being the case, then the required test period could be shortened.

The proposed test method will produce results that reflect insulation life relative to a reference refrigerant-lubricant mixture. Although Radian concluded that development of an absolute life prediction test is beyond the state of the art, the proposed SSU test method does represent a more economical test method than the battery of methods presently.used by the industry.

Figure 9-1. Stator Simulator Unit (SSU).

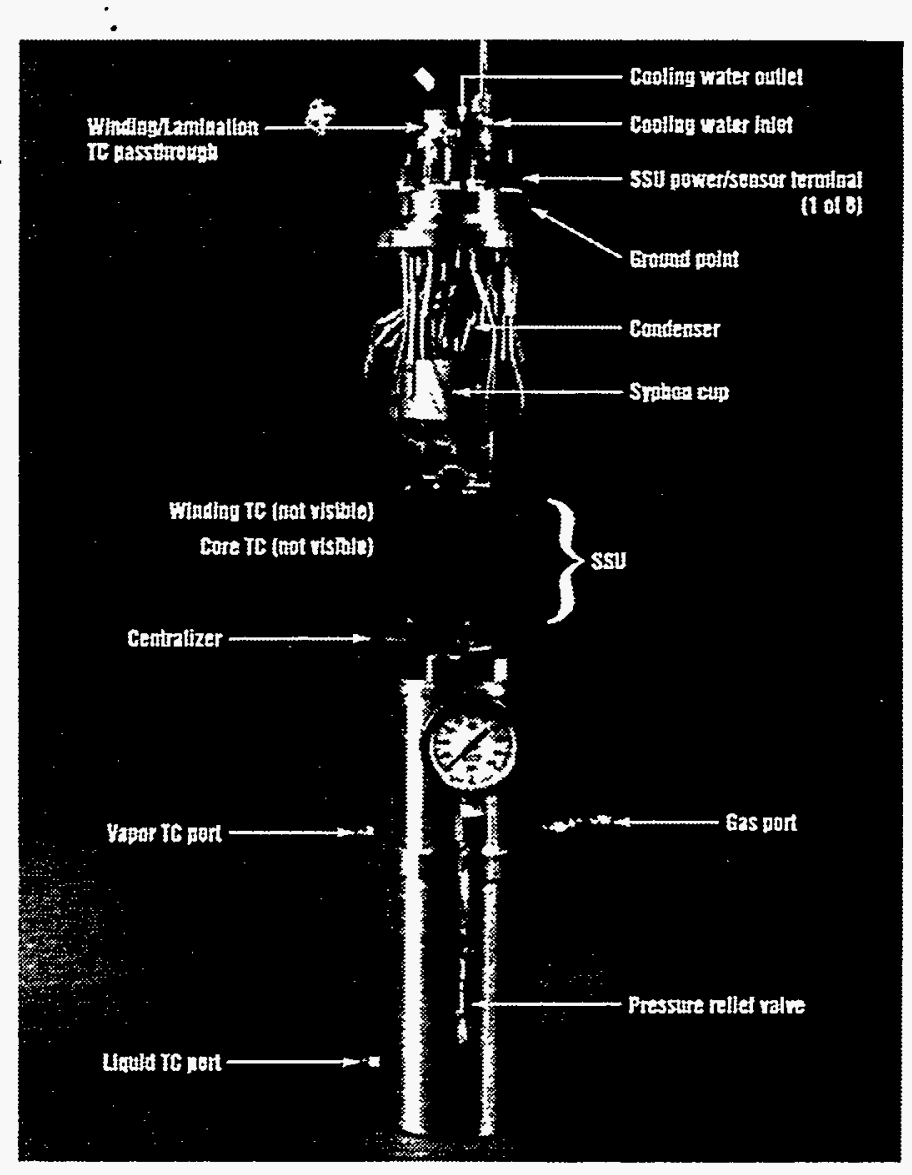




\section{ACCELERATED SCREENING METHODS \\ FOR DETERMINING CHEMICAL AND THERMAL STABILITY \\ OF REFRIGERANT-LUBRICANT MIXTURES}

\section{Objectives:}

To develop screening methods and procedures to assess the chemical and thermal stability of refrigerants and lubricants, as well as additives, metals, surface treatments, and polymers, used in hermetic systems.

To validate these screening methods and procedures.

\section{Results:}

This research is being performed by the University of Dayton Research Institute under contract to ARTI.

A literature search has been completed and several analytical techniques that might be developed into accelerated stability screening tests were identified. These methods employ one or more of the following techniques:

- Incorporation of thermocouple wells into sample vessels for temperature monitoring,

- In situ monitoring of temperature, conductivity, and/or voltage production,

- In situ monitoring of viscosity using surface acoustic wavelength devices,

- Employing differential thermal analysis (DTA) techniques during sample aging,

- Use of flat bottom, four millimeter diameter glass tubes for sample analysis,

- Use of miniature metal bombs for sample analysis.

The report, DOE/CE/23810-10, Accelerated Screening Methods for Determining Chemical and Thermal Stability of Refrigerant-Lubricant Mixtures; Part I: Method Assessment, by Robert Kauffman, April 1993, gives more details on the results of this literature search and the candidate screening methods. This report is currently available from the ARTI Refrigerant Database (RDB \#3501, 42 pages).

Part II concentrates on evaluating various techniques for development into an accelerated screening method. Details of the contractor's progress are contained in the draft final report, DOE/CE/23810-41, Accelerated Screening Methods for Determining Chemical and Thermal Stability of Refrigerant-Lubricant Mixtures; Part II: Experimental Comparison and Verification of Methods, by Mr. Robert Kauffman. 
Tests employing DTA techniques, using thermocouples or thermistors inside or outside the sample vessels, have been conducted. Initial results indicate that these techniques are only slightly sensitive to CFC-12/mineral oil reactions. It is hypothesized that these techniques will be less sensitive to HCFC/lubricant and HFC/lubricant reactions.

Use of ferric fluoride as a degradation catalyst was tested. Initial results show that at temperatures above $175^{\circ} \mathrm{C}\left(347^{\circ} \mathrm{F}\right)$, the catalyzed reactions appear to be more dependent on lubricant degradation than on refrigerant degradation. It is concluded that the use of . ferric fluoride as a catalyst may have the potential for development into an accelerated screening method for lubricant stability.

In situ color (light transmission) measurements were tested as a potential stability screening method. It was found that transmission depended on temperature and light source output, as well as color change of the refrigerant-lubricant mixture, and therefore may not be as promising as other screening techniques reviewed.

Tests involving in situ conductivity monitoring have also been performed. These techniques involve measuring current between two metal electrodes, sealed into the sample vessel, with a known applied voltage. Evaluations were made using combinations of: ac or DC voltage; tungsten, copper, and/or iron metal electrodes; steel, copper or no metal coupons as catalysts; and continuous or non-continuous conductivity monitoring. Initial results indicate that the in situ conductivity measurements correlate with refrigerantlubricant stability as reported in the literature and as determined by other analytical techniques (color and gas chromatography measurements). Initial results also show that continuous measurement of conductivity (i.e., maintaining the applied voltage throughout the aging process) accelerates as well as monitors the degradation of refrigerant-lubricant mixtures.

Initial tests were conducted using HFC-134a and four polyolester lubricants, heated in modified sealed glass tubes (see Figure 8-1) for two days at $175^{\circ} \mathrm{C}\left(347^{\circ} \mathrm{F}\right)$. Conductivity was monitored continuously by application of a triangular voltage wave-form (oscillating between \pm 15 volts) across two tungsten leads sealed into the tubes. Dramatic changes in the first several hours of measurements are hypothesized to be related to interactions between the metal (tungsten) surface and the refrigerant-lubricant mixture. Conductivity changes thereafter were seen to correspond to chemical/thermal stability as determined by ASTM color tests. 
Figure 8-1. Modified Sealed Glass Tube.

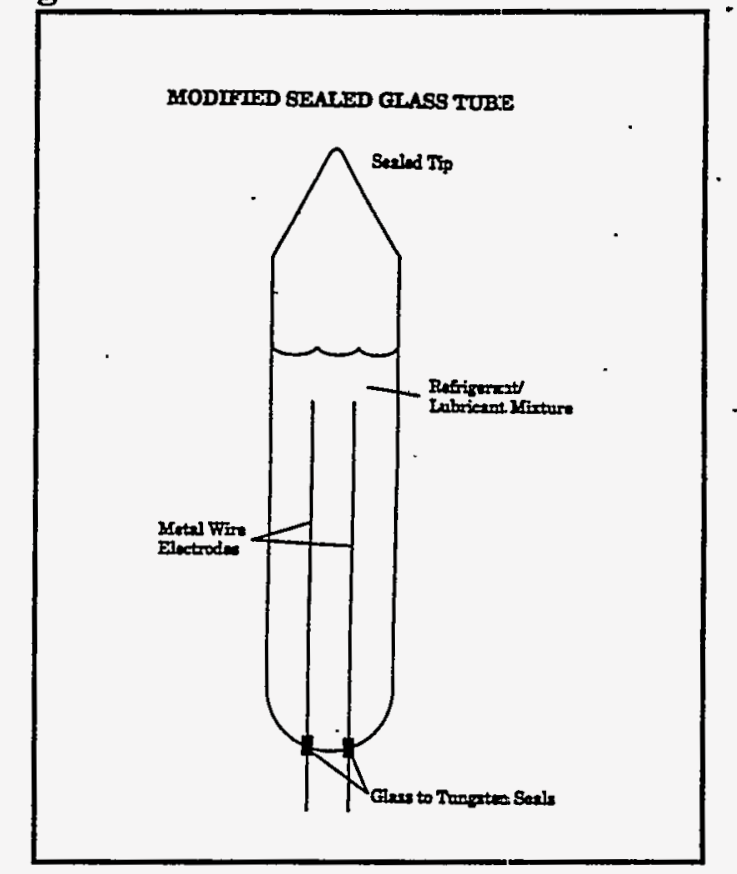

Three aluminum heating blocks (one with one well, one with three wells, and one with five wells) have been constructed with built-in cartridge heaters and electrical connections for monitoring the conductivity of the fluids inside the modified sealed tubes. A programmable temperature controller has been used to subject refrigerant/lubricant mixtures to both isothermal and ramped temperature tests. Figure 8-2, below, is a schematic of a three-well aluminum block heating system.

Figure 8-2. Three-Well Aluminum Block Heating System.

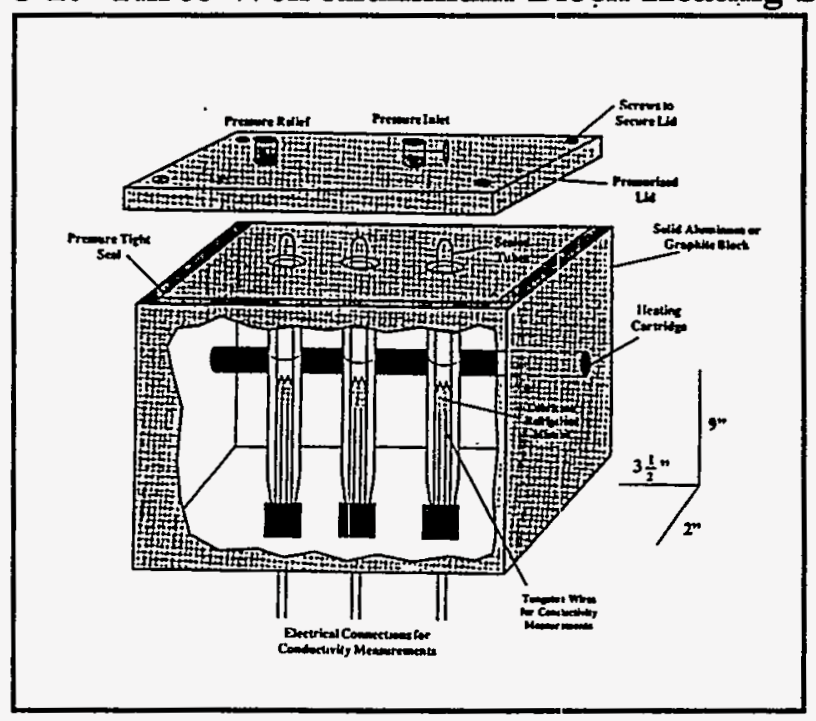


Tests have been conducted with refrigerant/lubricant mixtures in modified sealed glass tubes (see Figure $8-1$ above) at $175^{\circ} \mathrm{C}\left(347^{\circ} \mathrm{F}\right)$ for one week - half the time of the standard ASHRAE 97-1989 sealed glass tube tests. Refrigerants tested were CFC-12, HCFC-22, HFC-134a and a zeotropic blend of HFC-32/134a (30/70 by mass). Lubricants tested were a naphthenic and two paraffinic mineral oils (MOs), an alkylbenzene oil, two polypropylene glycols (PAGs) and four polyolesters (POEs). Conductivity was measured continuously by application of a $\pm 15 \mathrm{~V}$ triangular waveform ( 1 minute period) and graphs of conductivity (averaged over 1. hour intervals) vs. time were obtained.

The principal investigator has proposed that the following empirical relationship of the conductivity could be used to rank/screen the stability of the mixtures:

t

$$
\text { Total Conductivity Change }=\sum \mid \underset{n=0}{\mathrm{CR}_{\mathrm{n}+1}-\mathrm{CR}_{\mathrm{n}} \mid}
$$

where:

$\mathrm{CR}$ is the conductivity reading,

$\mathrm{n}=0$ is the time when the aluminum block reaches temperature equilibrium (approximately two hours),

$\mathrm{n}=\mathrm{t}$ is the time at which the total conductivity change is to be calculated, multiplied by the number of conductivity readings that are taken per unit time.

Total Conductivity Change calculations were prepared for the refrigerant/lubricant mixtures aged as described above. Other indices typically used to determine refrigerant stability, such as color and the presence of degradation products and trace metals, were also measured. The results are shown in Tables 8-1 through 8-4.

The principal investigator cites the following advantages for using in situ conductivity test as an accelerated screening method for determining chemical and thermal stability of refrigerant-lubricant mixtures:

- Good agreement with the current test as described in ANSI/ASHRAE Standard 97-1989, Sealed Glass Tube Method to Test the Chemical Stability of Material for Use Within Refrigeration Systems

- Less hazardous than ANSI/ASHRAE Standard 97-1989, due to elimination of handling of heated glass tubes

- More sensitive to degradation of high stability HCFC and HFC refrigerantlubricant mixtures than ANSI/ASHRAE Standard 97-1989

- Provides time resolved degradation measurements so that changes in degradation 
rates can be monitored in situ allowing for tests of unstable mixtures to be terminated early and for tests of highly stable mixtures to be extended

- Can be used to rapidly determine upper temperature limits of refrigerant-lubricant mixtures by applying increasing temperature profiles and monitoring conductivity in situ.

A final report is currently under review by the project monitoring committee. 
Table 8-1. Color, Volatile Degradation Products, Trace Metal, and Total Conductivity Measurements of CFC-12 Refrigerant/Lubricant Mixtures Aged at $175^{\circ} \mathrm{C}\left(347^{\circ} \mathrm{F}\right)$ for One Week

\begin{tabular}{|c|c|c|c|c|c|c|c|}
\hline \multirow{2}{*}{$\begin{array}{c}\text { Lubricant } \\
\text { (Note 1) }\end{array}$} & \multirow{2}{*}{$\begin{array}{c}\text { Steel } \\
\text { Catalyst? }\end{array}$} & \multicolumn{2}{|c|}{ Degradation } & \multicolumn{2}{|c|}{ Total Conductivity } & \multicolumn{2}{|c|}{ Trace Metals (ppm) } \\
\hline & & Color & Vol \% & 1 day & 7 days & $\mathbf{S i}$ & $\mathrm{Fe}$ \\
\hline Naphithenic MO & No. & $<05$ & $<0003$ & 20 & 25 & 4 & $<1$ \\
\hline Paraffinic \#1 MO & No & $<05$ & $<0 \cap 03$ & 20 & 20 & 5 & $<1$ \\
\hline Paraffinic \#2 $\mathrm{MO}$ & مa & $<05$ & $<0003$ & 2 & 5 & 3 & $<1$ \\
\hline Alkylhenzene & $\mathrm{No}$ & $<05$. & $<0003$ & 20 & 24 & 5 & $<1$ \\
\hline PAG-dial & No & $<05$ & مn1 & 664 & 1960 & 9 & $<1$ \\
\hline PAG-hutyl monoether & مم & $<05$ & $<002$ & Note 2 & Note 2 & 7 & $<1$ \\
\hline Mixed Acid \#1 POE & مNה & $<05$ & $<0003$ & 2 & 5 & 6 & $<1$ \\
\hline Mixed_Acid\#2POE & Nم & $<0.5$ & $0 \cap 03$ & 59 & 149 & 4 & $<1$ \\
\hline Branched_Acid \# 1 POE & Nم & $<05$ & $<0003$ & 66 & 536 & 6 & $<1$ \\
\hline Branched Acid \# 2 POE & $\mathrm{No}$ & $<05$ & $<0003$ & 4 & 10 & 28 & $<1$ \\
\hline Japhthenic MO & Yes & 15 & 031 & 16 & 84 & 25 & 3 \\
\hline Paraffinic \#1 MO & Yes & $<05$ & 003 & 1 & 6 & 4 & $<1$ \\
\hline Baraffinic \#2 MO & Yes & $<05$ & 009 & 2 & 6. & 8 & 1 \\
\hline Alkylhenzene & Yes & $<05$ & 008 & 6 & 15 & 11 & 1 \\
\hline PAG_dinl & Yes & Note 3 & Note 3 & Note 3 & Note 3 & Note 3 & Note 3 \\
\hline PAG - hutyl monoether & Yes & 780 & 231 & Note 4 & Note 4 & 964 & 58 \\
\hline Mixed_Acid \#1_POE & Yes. & $05-10$ & م96 & 209 & 1479 & 158 & 44 \\
\hline Mixed_Acid \#2 POE & Yes & 5.5 & 213 & Note 5 & Note 5 & 705 & 1640 \\
\hline Branched_Acid_\# 1 POE & Yes & 55 & 058 & Note 6 & Note 6 & 357 & 132 \\
\hline Branched_Acid \#2 2DEE & $\begin{array}{l}\text { Yes } \\
\text { (1) All lubri } \\
\text { (2) Heated } \\
\text { (3) Offscale } \\
\text { (4) Offscale } \\
\text { (5) Offscale } \\
\text { (6) Offscale }\end{array}$ & $\begin{array}{l}\text { a } 5 \\
\text { ats dried pric } \\
\text { only } 0.8 \text { day } \\
\text { ter } 0.1 \text { day t } \\
\text { on after heat } \\
\text { ter } 0.8 \text { days } \\
\text { ter } 0.4 \text { days }\end{array}$ & $\begin{array}{l}\frac{0.35}{\text { use }} \\
\text { exploded aft } \\
\text { then remov }\end{array}$ & $\begin{array}{l}216 \\
\text { days }\end{array}$ & 365 & 7 & 5 \\
\hline
\end{tabular}


Table 8-2. Color, Volatile Degradation Products; Trace Metal, and Total Conductivity Measurements of HCFC-22 Refrigerant/Lubricant Mixtures Aged at $175^{\circ} \mathrm{C}\left(347^{\circ} \mathrm{F}\right)$ for One Week

\begin{tabular}{|c|c|c|c|c|c|c|c|}
\hline \multirow{2}{*}{$\begin{array}{l}\text { Lubricant } \\
\text { (Note 1) }\end{array}$} & \multirow{2}{*}{$\begin{array}{c}\text { Steel } \\
\text { Catalyst } \\
\text { Used? }\end{array}$} & \multicolumn{2}{|c|}{ Degradation } & \multicolumn{2}{|c|}{$\begin{array}{c}\text { Total } \\
\text { Conductivity }\end{array}$} & \multicolumn{2}{|c|}{$\begin{array}{c}\text { Trace Metals } \\
\text { (ppm) }\end{array}$} \\
\hline & & Color & Vol. \% & 1 day & 7 days & Si & $\mathrm{Fe}$ \\
\hline Naphthenic MO & No & $0: 5$ & $<0.02$ & .149 & $\dot{284}$ & 92 & 1 \\
\hline Paraffinic \#1 MO & No & $<0.5$ & $<0.02$ & $: 4$ & 9 & 20 & $<1$ \\
\hline Paraffinic \#2 MO & No & $<0.5$ & $<0.02$ & 7 & 12 & 10 & $<1$ \\
\hline Alkylbenzene & No & 0.5 & $<0.02$ & 44 & 56 & 27 & 1 \\
\hline PAG - diol & No & $<0.5$ & $<0.02$ & 3654 & 23653 & 97 & $<1$ \\
\hline PAG - butyl monoether & No & 0.5 & $<0.02$ & 1595 & 2226 & 49 & $<1$ \\
\hline Mixed Acid \#1 POE & No & $<0.5$ & $<0.02$ & 286 & 591 & 23 & 1 \\
\hline Mixed Acid \#2 POE & No & $<0.5$ & $<0.02$ & 958 & 1330 & 9 & 1 \\
\hline Branched Acid \#1 POE & No & 1.5 & $<0.02$ & Note 2 & Note 2 & 32 & $<1$ \\
\hline Branched Acid \#2 POE & No & $<0.5$ & $<0.02$ & 32 & 38 & 49 & $<1$ \\
\hline Naphthenic MO & Yes & $<0.5$ & $<0.01$ & 61 & 68 & 21 & 1 \\
\hline Paraffinic \#1 MO & Yes & $<0.5$ & $<0.01$ & 5 & 9 & 62 & 2 \\
\hline Paraffinic \#2 MO & Yes & $<0.5$ & $<0.01$ & 10 & 15 & 9 & 2 \\
\hline Alkylbenzene & Yes & $<0.5$ & $<0.01$ & 66 & 90 & 23 & 2 \\
\hline PAG - diol & Yes & 05 & $0 . \dot{053}$ & Note 3 & Note 3 & 34 & 313 \\
\hline PAG - butyl monoether & Yes & 1.5 & $<0.01$ & 199 & 2525 & 23 & 852 \\
\hline Mixed Acid \#1 POE & Yes & $<0.5$ & $<0.01$ & 89 & 187 & 11 & 2 \\
\hline Mixed Acid \#2 POE & Yes & $<0.5$ & $<0.01$ & 631 & 1214 & 12 & 37 \\
\hline Branched Acid \#1 POE & Yes & 0.5 & $<0.01$ & Note 4 & Note 4 & 48 & 15 \\
\hline Branched Acid \#2 POE & Yes & $<0.5$ & $<0.01$ & 500 & 852 & 33 & 20 \\
\hline
\end{tabular}

Notes: (1) All lubricants dried prior to use

(2) Offscale after 3 hours

(3) Offscale after 0.5 days

(4) Offscale after 2.7 days 
Table 8-3. Color, Volatile Degradation Products, Trace Metal, and Total Conductivity Measurements of HFC-134a Refrigerant/Lubricant Mixtures Aged at $175^{\circ} \mathrm{C}\left(347^{\circ} \mathrm{F}\right)$ for One Week

\begin{tabular}{|c|c|c|c|c|c|c|c|}
\hline \multirow{2}{*}{$\begin{array}{c}\text { Lubricant } \\
\text { (Note 1) }\end{array}$} & \multirow{2}{*}{$\begin{array}{l}\text { Steel } \\
\text { Catalyst } \\
\text { Used? }\end{array}$} & \multicolumn{2}{|c|}{ Degradation } & \multicolumn{2}{|c|}{$\begin{array}{c}\text { Total } \\
\text { Conductivity } \\
\end{array}$} & \multicolumn{2}{|c|}{$\begin{array}{c}\text { Trace Metals } \\
\text { (ppm) }\end{array}$} \\
\hline & & Color & Vol. \% & 1 day & 7 days & Si & $\mathrm{Fe}$ \\
\hline Naphthenic MO & No & $<0.5$ & $<0.01$ & 1 & 3 & 49 & 1 \\
\hline Paraffinic \#1 MO & No & $<0.5$ & $<0.01$ & 2 & 9 & 9 & 1 \\
\hline Paraffinic \#2 MO & No & $<0.5$ & $<0.01$ & 1 & 7 & 8 & $<1$ \\
\hline Alkylbenzene & No & $<0.5$ & $<0.01$ & 1 & 7 & 7 & $<1$ \\
\hline PAG - diol & No & $<0.5$ & $<0.01$ & 4153 & 9102 & 13 & $<1$ \\
\hline PAG - butyl monoether & No & $<0.5$ & $<0.01$ & 237 & 494 & 10 & $<1$ \\
\hline Mixed Acid \#1 POE & No & $<0.5$ & $<0.01$ & 3 & 7 & 5 & $<1$ \\
\hline Mixed Acid \#2 POE & No & $<0.5$ & $<0.01$ & 144 & 288 & 8 & $<1$ \\
\hline Branched Acid \#1 POE & No & $<0.5$ & $<0.01$ & 47 & 276 & 7 & $<1$ \\
\hline Branched Acid \#2 POE & No & $<0.5$ & $<0.01$ & 2 & 8 & 7 & $<1$ \\
\hline Naphthenic MO & Yes & $<0.5$ & $<0.02$ & 1 & 2 & 8 & $<1$ \\
\hline Paraffinic \#1 MO & Yes & $<0.5$ & $<0.02$ & 1 & 6 & 15 & $<1$ \\
\hline Paraffinic \#2 MO & Yes & $<0.5$ & $<0.02$ & 1 & 3 & 9 & $<1$ \\
\hline Alkylbenzene & Yes & $<0.5$ & $<0.02$ & 1 & 3 & 22 & 1 \\
\hline PAG - diol & Yes. & $<0.5$ & $<0.02$ & 5548 & 18902 & 20 & 17 \\
\hline PAG - butyl monoether & Yes & $<0.5$ & $<0.02$ & 355 & 476 & 10 & 2 \\
\hline Mixed Acid \#1 POE & Yes & $<0.5$ & $<0.02$ & 25 & 43 & 10 & 5 \\
\hline Mixed Acid $\# 2$ POE & Yes & $<0.5$ & $<0.02$ & 163 & 336 & 9 & 2 \\
\hline Branched Acid \#1 POE . & Yes & $<0.5$ & $<0.02$ & 41 & 116 & 12 & 2 \\
\hline Branched Acid \#2 POE & Yes & $<0.5$ & $<0.02$ & 9 & 15 & 4 & $<1$ \\
\hline
\end{tabular}

Notes:

(1) All lubricants dried prior to use 
Table 8-4. Color, Volatile Degradation Products, Trace Metal, and Total Conductivity Measurements of HFC-32/134a (30/70) Refrigerant/Lubricant Mixtures Aged at $175^{\circ} \mathrm{C}\left(347^{\circ} \mathrm{F}\right)$ for One Week

\begin{tabular}{|c|c|c|c|c|c|c|c|}
\hline \multirow{2}{*}{$\begin{array}{l}\text { Lubricant } \\
\text { (Note 1) }\end{array}$} & \multirow{2}{*}{$\begin{array}{c}\text { Steel } \\
\text { Catalyst } \\
\text { Used? }\end{array}$} & \multicolumn{2}{|c|}{ Degradation } & \multicolumn{2}{|c|}{$\begin{array}{c}\text { Total } \\
\text { Conductivity }\end{array}$} & \multicolumn{2}{|c|}{$\begin{array}{c}\text { Trace Metals } \\
\text { (ppm) }\end{array}$} \\
\hline & & Color & Vol. \% & 1 day & 7 days & $\mathbf{S i}$ & Fe \\
\hline Naphthenic MO & No & $<0.5$ & $<0.02$ & 1 & 3 & 32 & $<1$ \\
\hline Paraffinic \#1 MO & No & $<0.5$ & $<0.02$ & 1 & -5 & 8 & $<\cdot 1$ \\
\hline Paraffinic \#2 MO & No & $<0.5$ & $<0.02$ & 1 & 6 & 16 & $<1$ \\
\hline Alkylbenzene & No & $<0.5$ & $<0.02$ & 1 & 3 & 15 & $<1$ \\
\hline PAG - diol & No & $<0.5$ & $<0.02$ & 4418 & 9700 & 26 & $<1$ \\
\hline PAG - butyl monoether & No & $<0.5$ & $<0.02$ & 332 & 1262 & 24 & $<1$ \\
\hline Mixed Acid \#1 POE & No ${ }^{-\cdot}$ & $<0.5$ & $<0.02$ & 1 & 6 & 11 & $<1$ \\
\hline Mixed Acid \#2 POE & No & $<0.5$ & $<0.02$ & 119 & 174 & 17 & $<1$ \\
\hline Branched Acid \#1 POE & No & $0.5-1.0$ & $<0.02$ & 14 & 109 & 11 & $<1$ \\
\hline Branched Acid \#2 POE & No & $<0.5$ & $<0.02$ & 8 & 17 & 17 & $<1$ \\
\hline Naphthenic MO & Yes & $<0.5$ & $<0.02$ & $<1$ & 4 & 13 & $<1$ \\
\hline Paraffinic \#1 MO & Yes & $<0.5$ & $<0.02$ & 1 & 5 & 10 & $<1$ \\
\hline Paraffinic \#2 MO & Yes & $<0.5$ & $<0.02$ & 1 & 6 & 15 & $<1$ \\
\hline Alkylbenzene & Yes & $<0.5$ & $<0.02$ & 1 & 3 & 14 & $<1$ \\
\hline PAG - diol & Yes & $<0.5$ & $<0.02$ & 3322 & 15148 & 21 & 13 \\
\hline PAG - butyl monoether & Yes & $<0.5$ & $<0.02$ & 465 & 565 & 7 & 3 \\
\hline Mixed Acid \#1 POE & Yes & $<0.5$ & 0.02 & 13 & 33 & 11 & $<1$ \\
\hline Mixed Acid \#2 POE & Yes & $<0.5$ & $<0.02$ & 389 & 851 & 12 & 6 \\
\hline Branched Acid \#1 POE & Yes & 0.5 & $<0.02$ & 54 & 197 & 64 & 2 \\
\hline Branched Acid \#2 POE & Yes & $<0.5$ & $<0.02$ & 20 & 35 & 15 & $<1$ \\
\hline
\end{tabular}

Notes:

(1) All lubricants dried prior to use 


\section{INVESTIGATION OF FLUSHING AND CLEAN-OUT METHODS}

\section{Objective:}

To develop one or more alternative flushing and clean-out procedures

- to effectively reduce mineral oil content in a HFC-134a retrofit to less than 5\% weight in polyolester lubricant or

- to successfully flush a refrigerant system after a compressor burnout has occurred, using a zero ozone depleting alternative flushing fluid.

Results:

Part 1 of this effort entailed a literature search and study of possible alternatives to the current flushing and cleanout methods. Part 2 of the project will prove out the feasibility of an alternative flushing and cleanout method. Two competing contractors were selected to conduct the Part 1 study. Following the review of both studies, ARTI awarded Part 2 to Integral Sciences, Inc.

A summary of the results of the literature search conducted by Integral Sciences, along with a description of proposed laboratory and field testing in contained in the interim report, DOE/CE/23810-37, Investigation of Flushing and Clean-Out Methods for Refrigerant Equipment to Ensure System Compatibility (Part 1), by John J. Byrne and Marc W. Abel, April 1994.

Integral Sciences will conduct laboratory and field testing to determining the effectiveness of using a low side oil separation and removal system for removing mineral oil during retrofit procedures. 


\section{INVESTIGATION INTO THE FRACTIONATION OF REFRIGERANT BLENDS}

\section{Objective:}

To develop theoretical models and verify with experimental data for:

- determining concentration and pressure shifts due to different solubilities of the refrigerant blend components in the lubricant, if any.

- investigating the effects of fractionation resulting from the successive system charges from a storage/shipping container on the performance of typical airconditioning unit.

- experimental verification of fractionation shifts in composition and pressure of zeotropic refrigerant blends within the components of a refrigeration system during operation and non-operation.

- experimental verification of fractionation shifts in composition and pressure resulting from slow (isothermic) and rapid (adiabatic) leak scenarios.

\section{Results:}

United Technologies Research Center (UTRC) is performing this research under contract to ARTI. A detailed report of its work to date is included in the quarterly technical status report, DOE/CE/23810-51C, Investigation into the Fractionation of Refrigerant Blends, by Frank Biancardi, January 1995. Theoretical models are under development for each of the scenarios listed in the objective. Once formulated, these models will be verified by comparing predicted compositions from the model with experimental measurements in actual systems. 


\section{METHODS DEVELOPMENT}

FOR MEASURING AND CLASSIFYING

FLAMMABILITY/COMBUSTIBILITY OF REFRIGERANTS

\section{Objectives:}

To develop appropriate test procedures and conditions, based on an understanding of ANSI/ASTM E681-85, to measure the flammability of refrigerants.

To establish the conditions under which refrigerants and refrigerant blends exhibit flammability and/or combustibility, as a function of composition and test conditions including the effects of humidity.

\section{Results:}

The New Mexico Engineering Research Institute (NMERI), University of New Mexico is performing the work under contract to ARTI. To date NMERI has completed a literature search of technical papers on flammability test methods, summarized their conclusion and developed an annotated bibliography of these technical papers. The results of this initial effort are documented in the interim report, DOE/CE/23810-42G, Methods Development for Measuring and classifying Flammability/Combustibility of Refrigerants: Task 1 - Annotated Bibliography and Summary, by Everett W. Heinonen and Robert E. Tapscott, June 1994. NMERI also incorporated this information in a PC based flammability refrigerants database.

Building on the knowledge gained from Task 1, NMERI developed a test plan to investigate the effects of various parameters that effect a refrigerants flammability using a stainless steel explosion sphere test rig and a glass sphere as prescribed by an ASTM Standard E-681. The stainless steel explosion sphere uses pressure rises and rates of pressure rise for detection of flammability/combustibility, while the glass flask uses visual observation for detection. Results are reported in the draft final report, DOE/CE/2381050, Methods Development for Measuring and Classifying Flammability/Combustibility of Refrigerants: Task 3 - Laboratory Test Results, by Everett W. Heinonen and Robert E. Tapscott, December 1995.

The effect of four different ignition sources (electrical activated match head, electrically heated wire, AC spark and DC spark), initial pressure, initial temperature, and humidity on the flammability limits of propane and a number of refrigerants including R-32, $\mathrm{R} 32 / 125, \mathrm{R} 32 / 134 \mathrm{a}$, and $\mathrm{R} 32 / 134 \mathrm{a} / 125$ were investigated in both test apparatuses. 
NMERI reported the following conclusions:

Visual observations of flammability with R-32 and blends containing R-32 corresponded to overpressures of slightly over $2.1 \mathrm{kPa}(0.3 \mathrm{psi})$. However, corresponding overpressures for other flammability refrigerants are likely to vary.

The match ignitions resulted in lower concentrations observed for the lower and upper flammability limits (LFL and UFL) compared to those measured using other ignition sources.

The match ignitions resulted in lower concentrations observed for the lower and upper flammability limits (LFL and UFL) compared to those measured using other ignition sources.

DC spark ignition sources developed for the test were less than satisfactory. Lowvoltage DC spark ignition source delivered less energy to the spark gap than anticipated and the high-voltage DC spark ignition source had an incident in which and electrical arc was generated outside the test apparatus.

The AC spark ignition source was an effective ignitions source which repeatedly ignited refrigerant blends.

The heating wire ignition source provided enough energy to ignite propane, but not enough to ignite $\mathrm{R}-32$.

Higher initial pressures created higher overpressures.

Higher initial temperatures widened the flammability limits of R-32 blends. However, the presence of humidity is even greater.

The relative effects of initial temperature (ambient versus $100^{\circ} \mathrm{C}$ ) and humidity (dry versus moist) are dramatically illustrated in Figures 11-1 through 11-3 for three different test refrigerant blends containing $\mathrm{R}-32$. 
Figure 11-1. R32/134a Refrigerant Blend Flammability

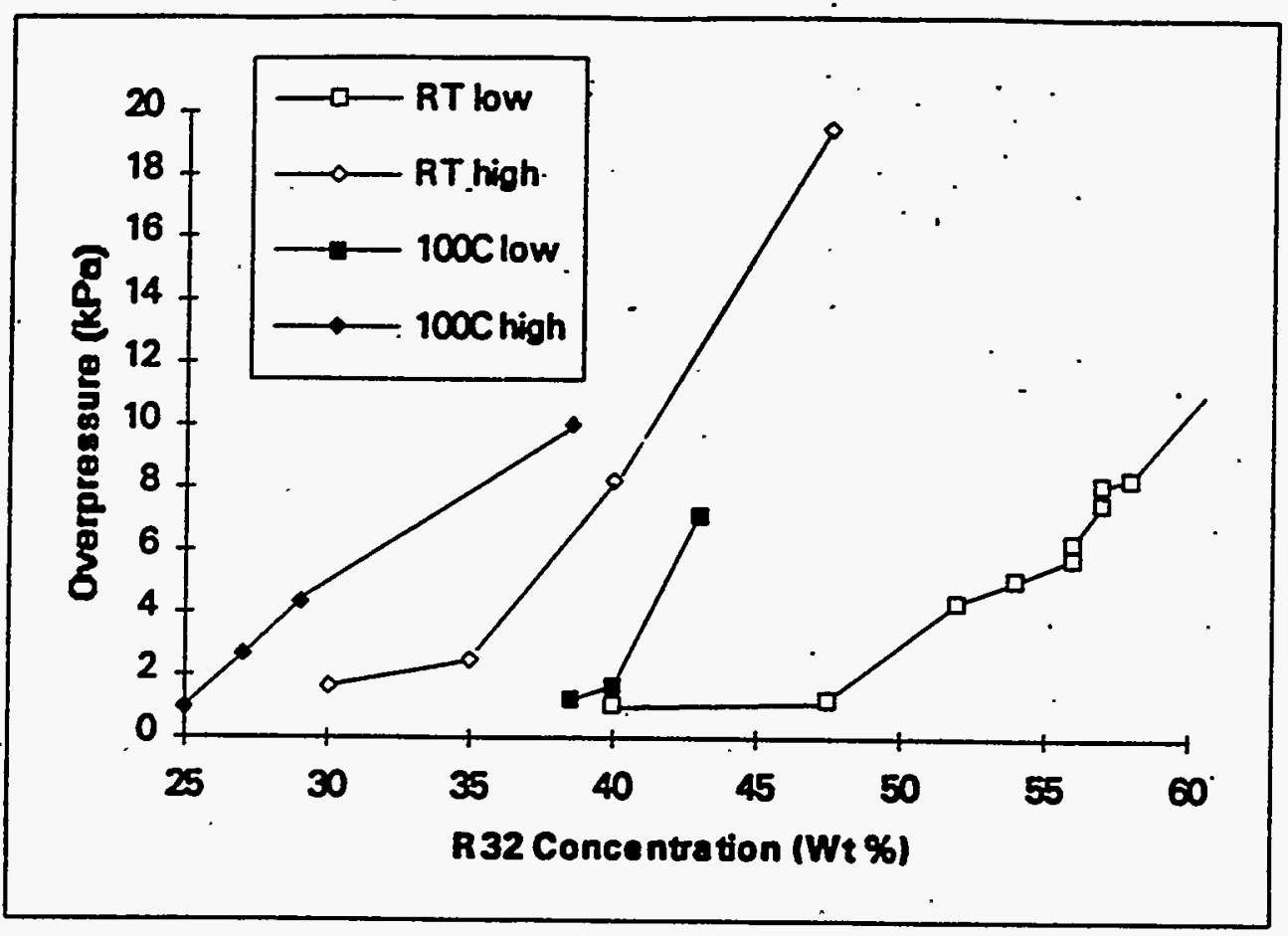

Figure 11-2. R32/125 Refrigerant Blend Flammability

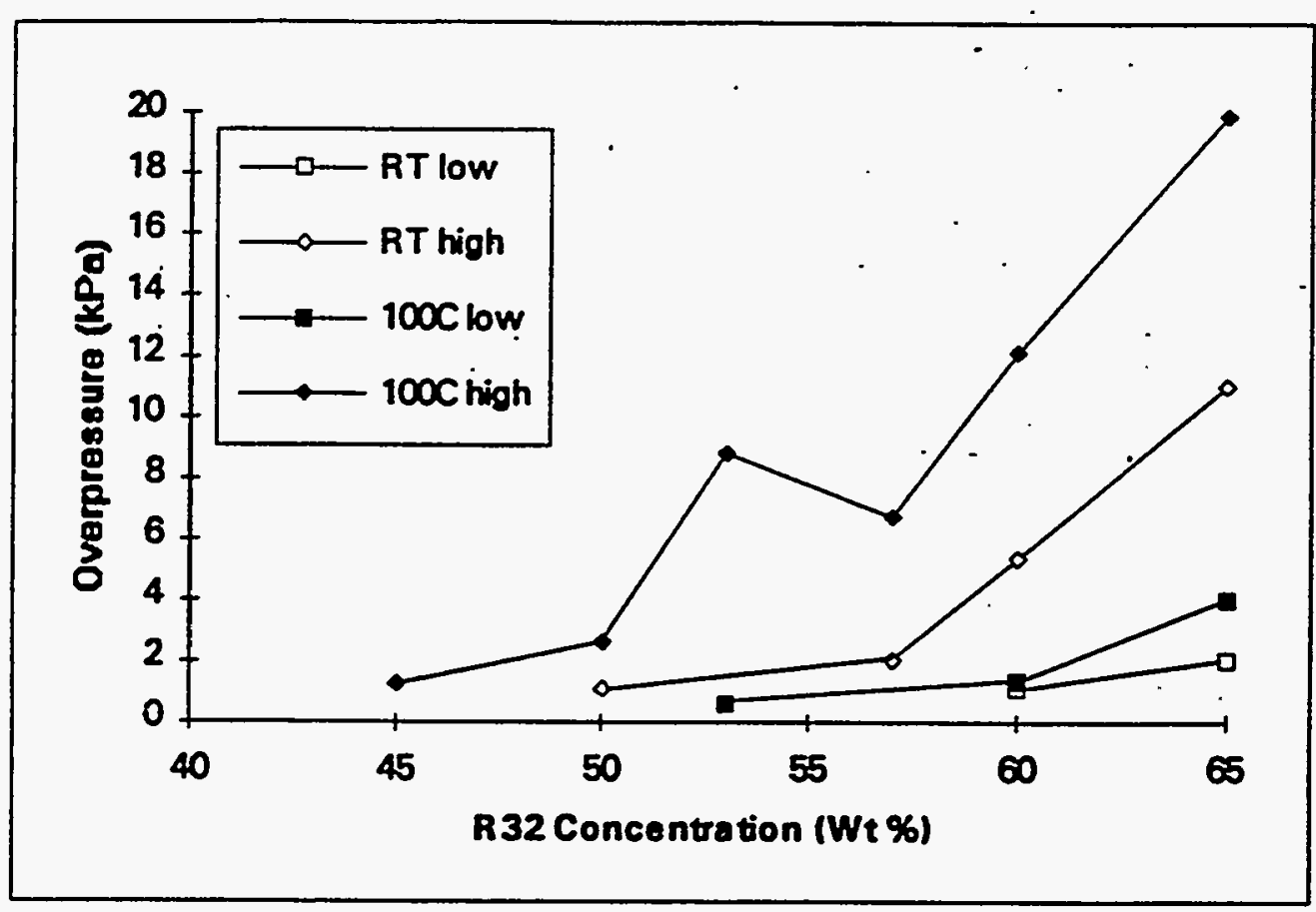

$11-3$ 
Figure 11-3. R32/134a/125 Refrigergnt Blend Flammability

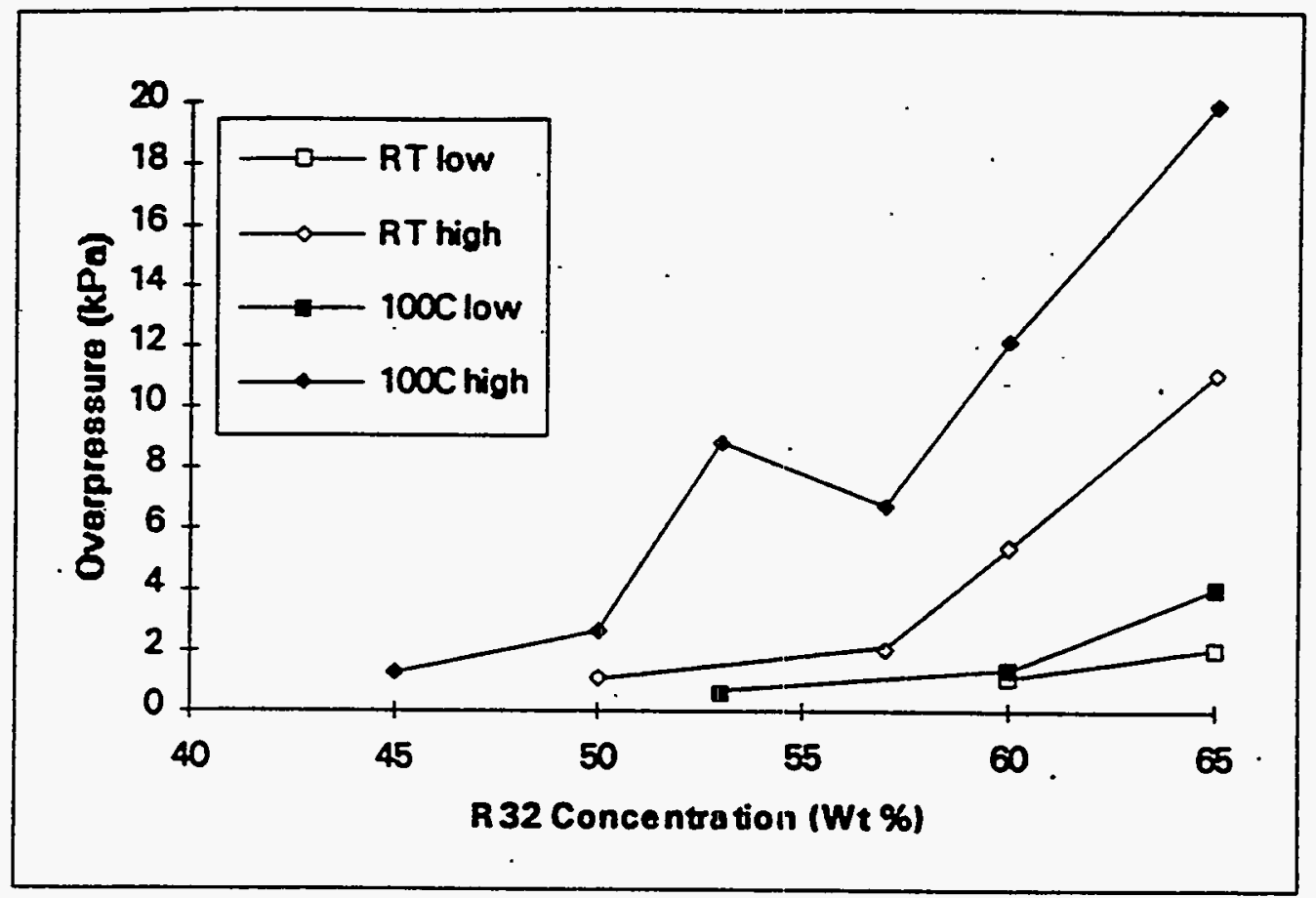

$11-4$ 


\section{Objective:}

To evaluate the suitability of an opposed-flow twin-flame burner for determining flammability limits of refrigerants.

\section{Results:}

The Building and Fire Research Laboratory of the National Institute of Standards and Technology (NIST) is performing this project under contract to ARTI. Phase I of this project used a opposed-flow burner (see Figure 12-1) to evaluate the flammability limits of methane, HFC-32 and mixtures of HFC-32 and HFC-125 in air for different flow conditions. NIST has completed Phase I and has published its results in the interim report, DOE/CE/23810-58, Lean Flammability Limit as a Fundamental Refrigerant Property, by C. Womeldorf, M. King and W. Grosshandler, 31 March 1995.

\section{Figure 12-1. Opposed-flow Burner}

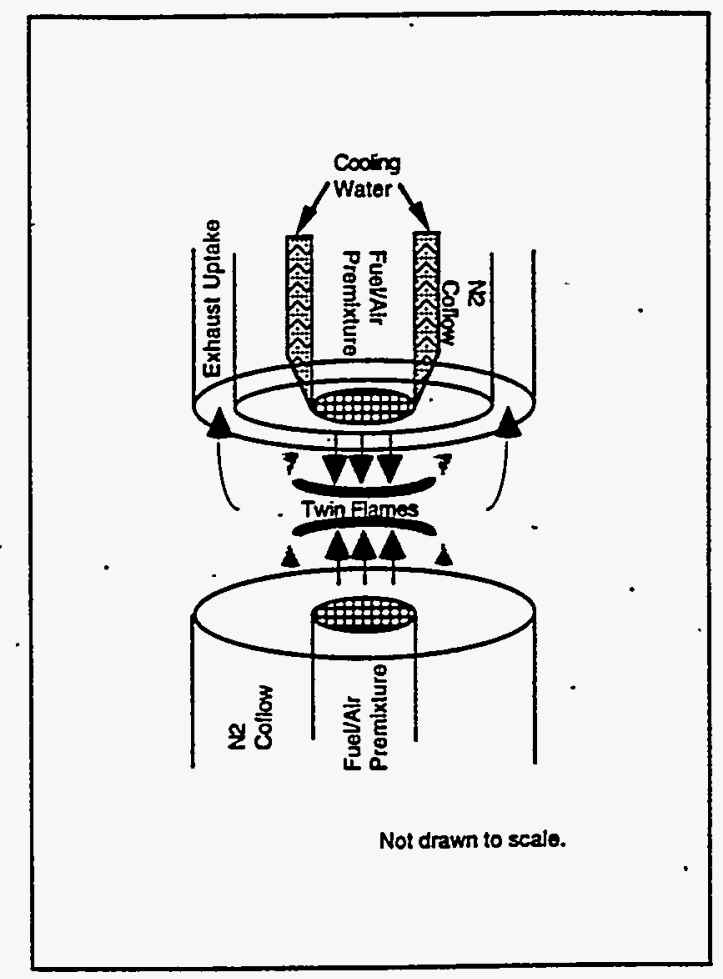

Eiqure 1: Flow diagram of counterflow bumer. 
- Demonstration of Apparatus Suitability

The lean flammability limit is defined as the fuel/air mixture which extinguishes an adiabatic flame when the strain rate (i.e., the norrnal gradient of velocity) is zero. The lean flammability limit can be evaluated at either the upper or lower flammability limits by extrapolating extinguishment stoichiometries for decreasing strain rates to zero. This method was described by Law, Zhu and Yu (1986), "Propagation and Extinction of Stretched Premixed Flames", 21st Symposium on Combustion: The Combustion Institute, pages 1419-1426.

As a result of Phase I work, NIST confirmed the suitability of the opposed-flow burner for evaluating lean flammability limits and established that the lean flammability limits of refrigerants and refrigerant mixtures could be evaluated in the opposed-flow burner apparatus.

From measurements using the opposed-flow burner, NIST estimated the lean flammability limit at the lower flammability limit of R-32 to be $12.0 \pm 0.6 \%$ by volume in air and at the upper flammability limit around $30 \%$ R-32 by volume in air (insufficient data points were taking at the upper flammability limit to determine a range). Figure 12-2 plots the measured extinction points of R-32/air premixed flames in terms of global strain rate versus equivalence ratio. A scale for $\% \mathrm{R}-32$ by volume in air corresponding to the equivalence ratio is also included.

\section{Figure 12-2. Extinction Points of R-32/Air Premixed Flame}

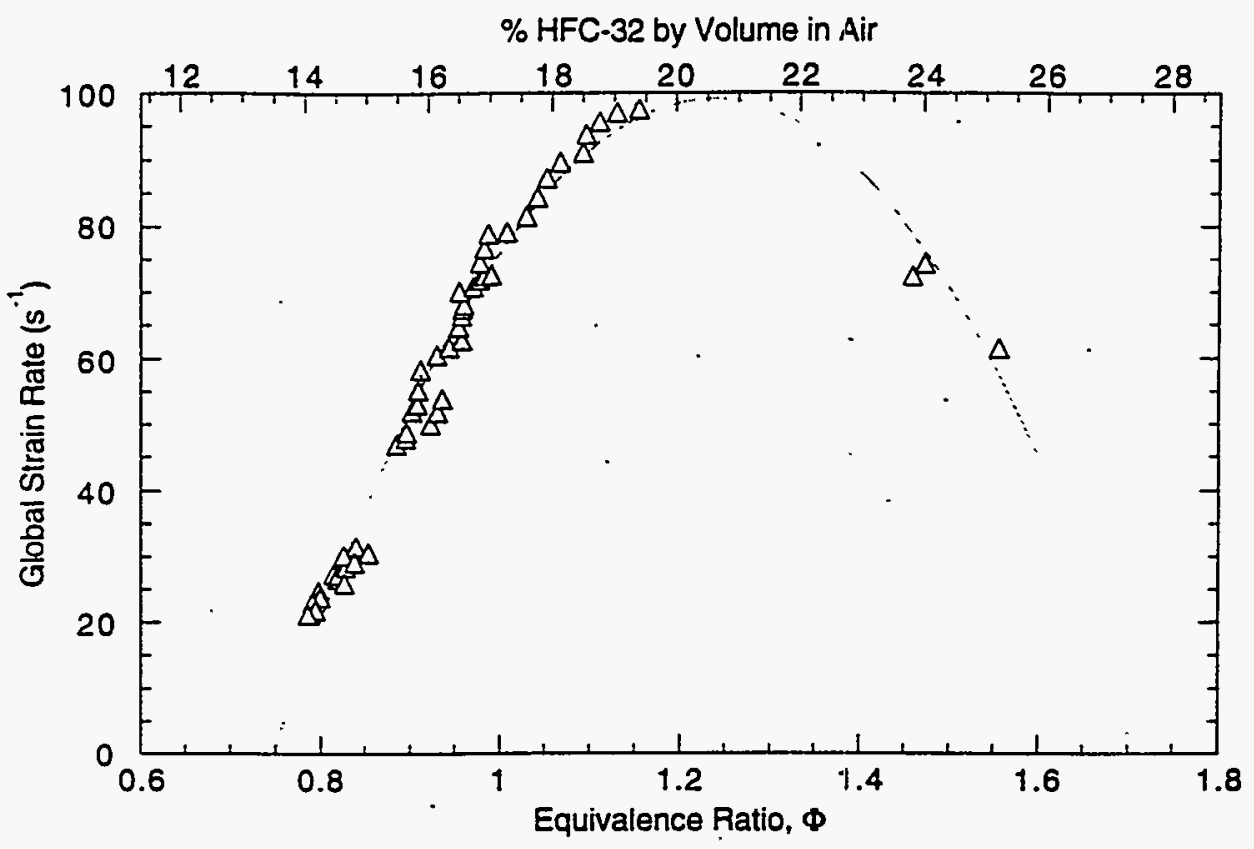

$12-2$ 
Global strain rate is defined as the mean incoming jet velocity divided by the half the jet separation. This neglects any thickness of the stagnation layer between the twin flames. For its measurements of R-32 and R-32/125 mixtures, NIST keep the jet separation distance constant at $15.9 \pm 0.1 \mathrm{~mm}(0.625 \mathrm{in})$ while varying the velocity below $200 \mathrm{~cm} / \mathrm{s}$ $(6.6 \mathrm{ft} / \mathrm{s})$. NSST noted that repeatable results could not.be achieved at velocities less than $15 \mathrm{~cm} / \mathrm{s}\left(0.5^{\circ} \mathrm{ft} / \mathrm{s}\right)$ due to buoyancy effects destablize the stagnation plane and the flame.

The equivalence ratio, $\Phi$, is defined as the number of moles of R-32 (or for mixtures the number of mole of R-32 plus R-125) per mole of air, normalized by the stoichiometric fuel/air molar ratio. For R-32 and refrigerant mixtures containing R-32 as the flammable component, the equivalence ratio can be converted to \% volume refrigerant using the following formula:

$$
\text { percent volume of refrigerant }=100\left(\frac{\Phi}{\Phi+4.76}\right)
$$

Figure 12-3 depicts the linear fits that NIST applied to data subsets to determine the lean flammability limit. As shown in Figure 12-4, these values within the range of published values.

Figure 12-3. Range of Lean Flammability Limit of R-32 in Air

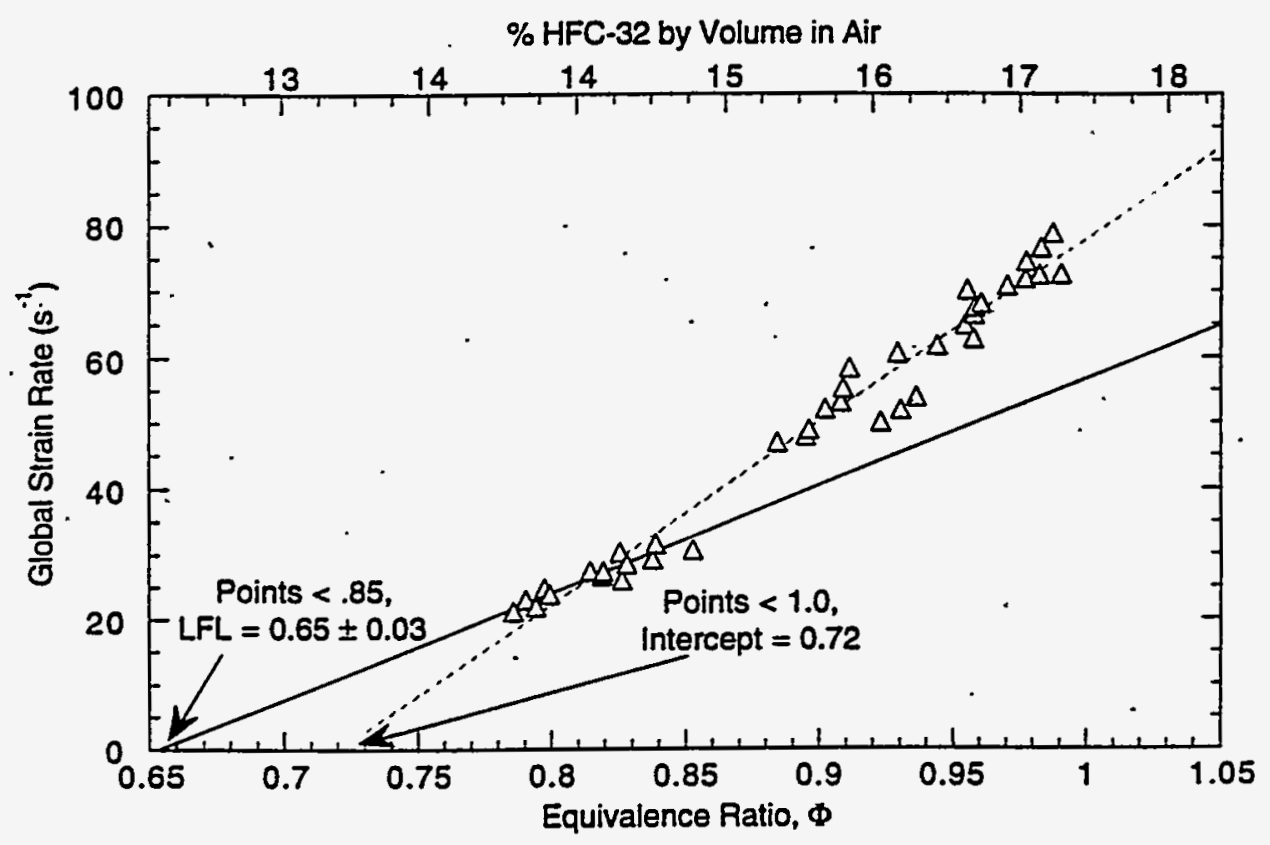

$12-3$ 
Figure 12-4. Comparison R-32 Lean Flammability Kimit with Other Published Results

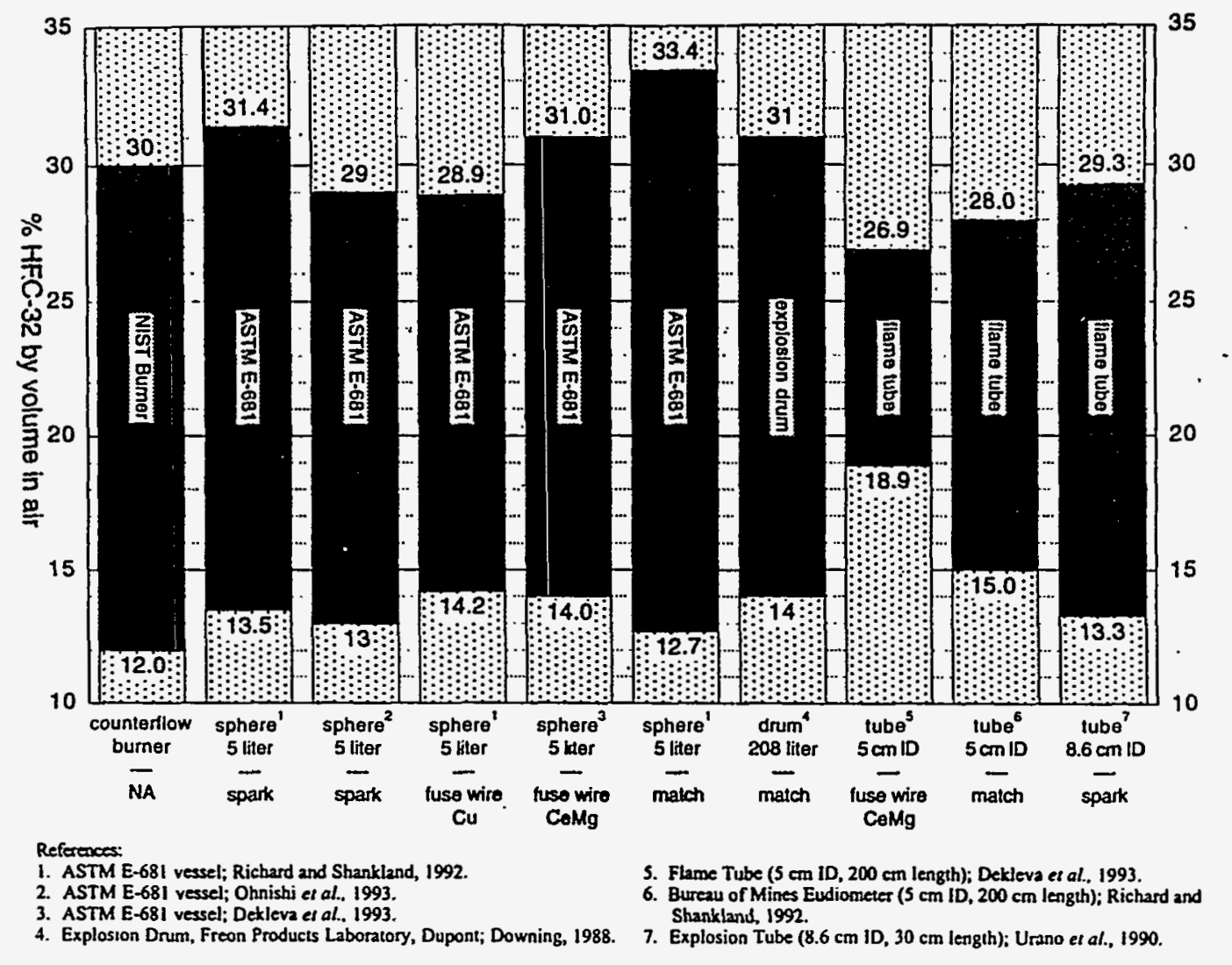

NIST also used the opposing-flow burner to determine the critical flammability ratio of R125 in R-32 and estimated it to be $18.5 \pm 0.8 \%$ by volume. Figure $12-5$ plots the measured values for the global strain rate versus the equivalence ration and Figure 12-6 plots NIST's extrapolation of this data to produce the critical flammability ratio.

Results from these experiments are preliminary and were used strictly to evaluate the feasibility of this type apparatus. In Phase 2 of this project, NIST will make design improvements to the opposing-flow burner apparatus and will ascertain operating parameters of the new apparatus by conducting measurements on R-32/dry air and methane/dry air. NIST will also conduct measurements to determine the lean flammability limits of R-32/air, R-134a/air , and R-245ca/air at $0 \%$ and $50 \%$ relative humidity air (humidity level of air at room temperature and atmospheric pressure). 
Figure 12-5. Extinction Points for R-32/125 Flames at Various Concentrations of R-125

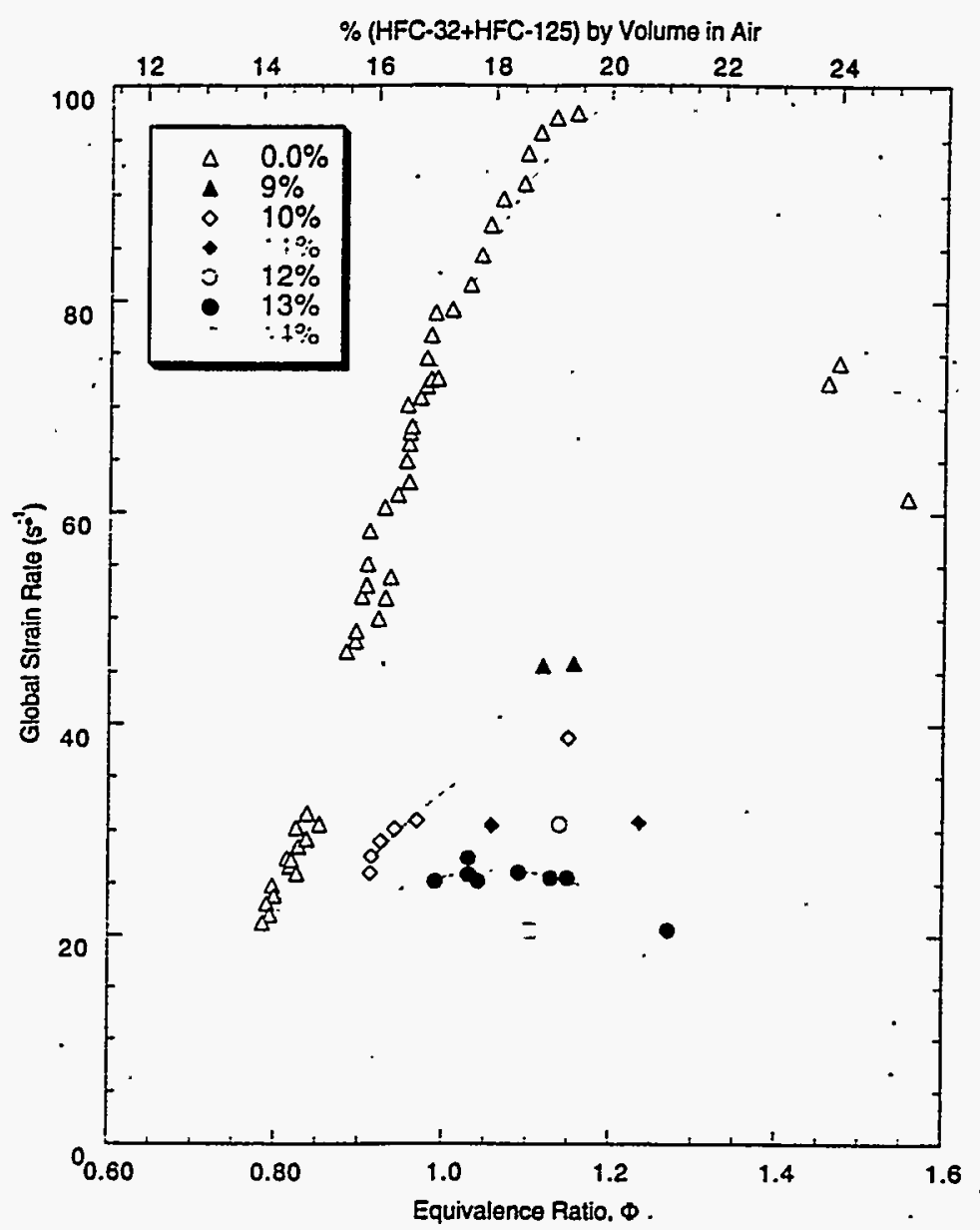

Figure 12-6. Extrapolation of Zero Strain Rate to Determine Critical Flammability Ratio of R-125 in R-32

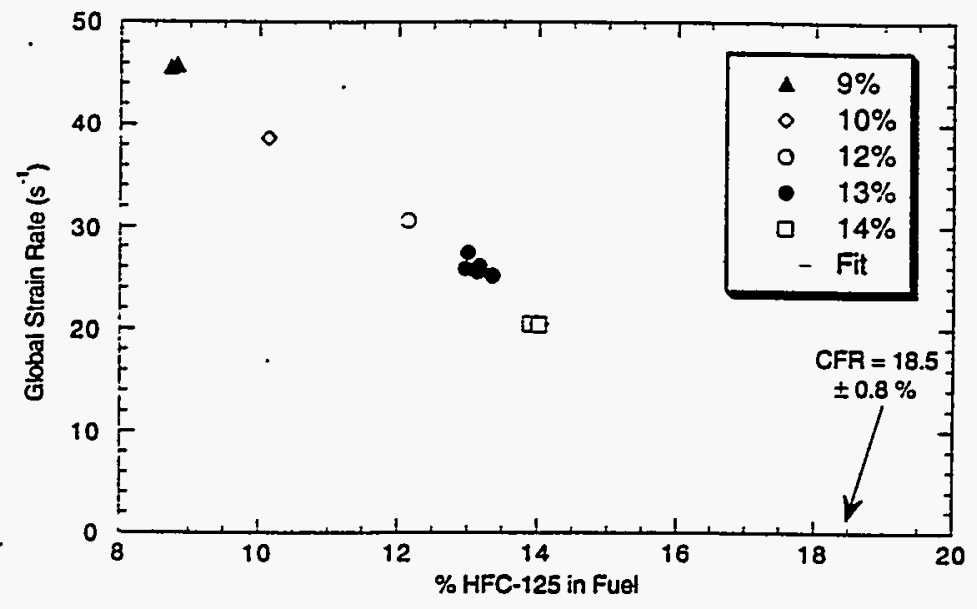

$12-5$ 


\section{Objective:}

To provide information that will enable manufacturers of refrigeration components and systems to select reliable POE lubricant additives.

\section{Results:}

Imagination Resources, Inc., is performing this research under contract to ARTI.

Part I of this project consists of a confidential survey of the lubricant additives being used in the commercial production of suitable refrigerant polyolesters. It is unlikely that specific additives will be identified due to the competitive nature of this information. Nonetheless, it is anticipated that the general chemical category or class of the substance, as well as its purpose, will be revealed. The contractor is currently conducting research under Part I of this project.

Compatibility and stability tests will be conducted under Part II of this project. 


\section{STUDY OF FOAMING CHARACTERISTICS}

\section{Objective:}

To determine the rate at which a POE lubricant will. absorb HFC refrigerants.

To determine the rate at which an HFC refrigerant leaves solution with a POE when exposed to a pressure drop.

To define the characteristics of the foam formed when refrigerant leaves solution with a lubricant after being exposed to a pressure drop.

\section{Results:}

Proposals for performing research to meet the above objectives have been received and are under evaluation. A contractor has not yet been picked. 


\section{STUDY OF LUBRICANT CIRCULATION IN SYSTEMS}

\section{Objectives:}

Determine the fundamental lubricant return parameters for HFC/POE mixtures to map how the characteristics of different refrigerant/lubricant mixtures affect lubricant return to the compressor.

Determine the fundamental lubricant return parameters for $\mathrm{HFC} /$ mineral oil mixtures to assess whether (or under what circumstances) irnmiscible systems can provide sufficient lubricant return.

Results:

The United Technologies Research Center (UTRC) was recently placed under contract with ARTI to perform this work. An interim progress report is expected in October 1995.

The overall purpose of the project is to investigate the impact of fluid properties, for immiscible refrigerant-lubricant mixtures, on lubricant return in typical residential splitunit systems. UTRC will identify worst case (i.e., extreme limits that manufacturers permit their equipment to be installed and operated) vertical and horizontal separation (example: $20 \mathrm{ft}$ vertical and $100 \mathrm{ft}$ horizontal), operating temperatures (example: heat pump winter operation of $0^{\circ} \mathrm{F}$ ), and typical failure modes that could jeopardize the system in heating or cooling modes.

To make the testing insensitive of the compressor utilized (e.g., in general, a scroll compressor puts out much less lubricant than does a reciprocating compressor), a known amount of lubricant will be injected into the compressor discharge stream. The refrigerant/lubricant pairs to be tested in the work are:

Baselines Mixtures R-22/Suniso3GS mineral oil

$\mathrm{R}-407 \mathrm{C} /$ Suniso 3GS mineral oil

$\mathrm{R}-407 \mathrm{C} /$ Suniso $1 \mathrm{GS}$ mineral oil

$\begin{array}{llll}\text { Test Mixtures } & \text { R-407C/Mobil EAL 22C } & \text { low miscibility } & \text { low viscosity } \\ & \text { R-407C/ICI RL68L } & \text { low miscibility } & \text { high viscosity } \\ \text { R-407C/Castrol SW22 } & \text { high miscibility } & \text { low viscosity } \\ & \text { R-407C/Castrol SW68 } & \text { high miscibility } & \text { high viscosity }\end{array}$ 
For each of the seven refrigerant-lubricant pairs indicated above, four separate test modes will be run:
(1) worst case heating
(2) worst case heating with a component failure (e.g., blocked fan)
(3) worst case cooling
(3) worst case cooling with a component failure (e.g., blocked fan) .

Each of the above tests will be conducted to ensure failure. The tests will be performed in duplicate to improve accuracy of results. On-line, dynamic instrumentation will be utilized to measure oil circulation and to ascertain whether the lubricant is being moved as a slug or as interspersed liquid. 


\section{Objectives:}

Model the performance of HFC-245ca in actual chillers.

Conduct performance tests of HFC-245ca in a low pressure chiller and compare the results with the modeled performance and with performance tests of CFC-11 and HCFC-123 in the same chiller.

Assess the commercial viability of HFC-245ca to retrofit CFC-11 and HCFC-123 chillers in the field and for use in new chillers.

\section{Results:}

The Trane Company was recently placed under contract with ARTI to perform this work. As indicated by the objectives, the work is to accomplished in three tasks:

\section{Task 1 - Calculate Performance}

The cycle performance of a chiller utilizing HFC-245ca will be calculated and compared to the modeled results for CFC-11 and for HCFC-123.

\section{Task 2 - Performance Tests}

The relative heat transfer performance of HFC-245ca, HCFC-123, and CFC-11, in both the boiling and condensing modes, will be evaluated in single-tube tests for two different tube geometries.

The capacity and efficiency, at ARI water-cooled conditions, will be determined in a low-pressure chiller operated with HFC-245ca. The results will be compared against baseline performance tests obtained with CFC-11 and HCFC-123.

\section{Task 3 - Commercial Viability}

Assess the commercial viability for use of HFC-245ca to retrofit CFC-11 and HCFC-123 chillers in the field and for use in new chillers (assuming acceptable toxicity results).

An interim progress report detailing the results from the Task 1 modeling is expected to be available for release in June 1995. The final report is expected to be available for release in March 1996. 
REFRIGERANT DATABASE

\section{Objectives:}

To develop a database for materials compatibility and lubricant research (MCLR) information on substitutes for chlorofluorocarbon (CFC) and hydrochlorofluorocarbon (HCFC) refrigerants for applied refrigeration cycles.

To assemble physical properties, materials compatibility, and related test data for these refrigerants and lubricants, along with comparative data for currently-used refrigerants.

To make the data readily accessible for rapid screening and identification of pertinent source documents based on user-defined search criteria.

\section{Results:}

James M. Calm, Engineering Consultant, is performing this research under contract to ARTI. The database is available on a subscription basis (for a nominal charge to recover distribution costs) in either a computerized or printed format.

The core of the database consists of bibliographic citations and synopses for publications that may be useful in research and design of air-conditioning and refrigeration equipment. The bibliographic citations provide information to facilitate ordering of source documents from the author or the publisher. Approximately $40 \%$ of the documents are available from the database contractor. Detailed synopses have been prepared for many of the entries. These detailed synopses describe the data, tests, evaluations, and the materials noted in the documents. The synopses permit searching of information by refrigerant or refrigerantlubricant combination, topic, author, material (by generic or commercial name), specific refrigerant property, or just about any other combination of search criteria.

The computerized version of the database includes summaries for over 240 refrigerants, both single-component and blends. Refrigerants are identified by ASHRAE Standard 34 designations, chemical names and formulae, common names, refrigerant groups, blend compositions, and familiar chemical abstract numbers. Summary property data (with dimensional quantities in dual IP and SI units) are provided for molecular mass, atmospheric boiling point, melting or freezing point, and critical-point parameters. The lower and upper flammability limits (LFL and UFL), ASHRAE Standard 34 safety classification, ozone depletion potential (ODP), global warming potential (GWP), halocarbon global warming potential (HGWP), and common uses are indicated if known. Specific sources are referenced for the data to enable verification, obtaining further information, and examining underlying limitations. 
The February 1995 release of the ARTI Refrigerant Database contained in excess of 2,600 entries related to:

- refrigerant properties

- performance with new refrigerants

- materials compatibility

- lubricants for new refrigerants

- environmental and safety data

- related research programs

- toxicity data 


\section{REFRIGERANT TOXICITY SURVEY}

\section{Objective}

This research project entails search, review, and consolidation of toxicity information on alternative refrigerants as well as development of recommendations for toxicity classification methods for air-conditioning and refrigeration applications. The work addresses four needs:

- To locate and assemble data on new refrigerants for classification and determination of allowable quantities in safety standards.

- To prepare a summary, with referenced data, on the health effects of new refrigerants for use by the air-conditioning and refrigeration industry in assessing refrigerant toxicity.

- To incorporate the data and identified sources (references) into the ARTI Refrigerant Database, to facilitate subsequent retrieval of information needed to satisfy building code requirements.

- To provide recommendations to improve the manner by which toxicity is classified.

\section{Results}

James M. Calm, Engineering Consultant, is performing this research under contract to ARTI. Results from the work are expected second quarter 1995 


\section{THERMOPHYSICAL PROPERTIES OF HFC-32, HCFC-123, HCFC-124 AND HFC-125}

\section{Objective:}

To provide highly accurate, selected thermophysical properties data for refrigerants HFC$32, \mathrm{HCFC}-123, \mathrm{HCFC}-124$, and HFC-125; and to fit these data to theoretically-based equations of state and detailed transport property models.

\section{Results:}

The Thermophysics Division of the National Institute of Standards and Technology (NIST) has completed measurements and correlations of HFC-32, HCFC-123, HCFC-124 and HFC-125. This data filled the gaps that existed. in data sets and resolved problems and uncertainties that existed in and between those data sets. Measurements and determinations of thermodynamic properties included vapor pressure-volume-temperature behavior, liquid pressure-volume-temperature behavior, saturation and critical points, vapor speed of sound and ideal gas heat capacity, and isochoric heat capacity. The data was fitted to the Carnahan-Starling-DeSantis (CSD) and the modified Benedict-WebbRubin (MBWR) equations of state. Measurements and correlations of transport properties included thermal conductivity and viscosity measurements.

A detailed report of the results is presented in the final report, DOE/CE/23810-16, Thermophysical Properties, April 1993, by Richard F. Kayser, PhD (RDB \#3860, 242 pages). Key results are summarized below:

\section{$\underline{\mathrm{HFC}-32}$}

\section{MBWR Equation of State}

NIST has revised its 32-term MBWR equation of state and its ideal gas heat capacity $\left(\mathrm{C}_{\mathrm{p}}^{\circ}\right)$ equation for HFC-32 (see Table 19-1). The equation is reported to be valid at temperatures from the triple point at $137 \mathrm{~K}$ up to $400 \mathrm{~K}\left(-213\right.$ to $\left.260^{\circ} \mathrm{F}\right)$. The maximum pressure for the equation is $40 \mathrm{MPa}(5800 \mathrm{psi})$. The equation may be reasonably extrapolated up to $500 \mathrm{~K}\left(440^{\circ} \mathrm{F}\right)$ and $100 \mathrm{MPa}(14500 \mathrm{psi})$. NIST fitted the equation using a multi-parameter linear least squares routine on the measured data. 


\section{Molar Heat Capacity}

The molar heat capacity of HFC-32 was measured using an adiabatic calorimeter. Measurements included 79 values in the liquid state and 105 values in the vapor and liquid two-phase region. The measurements covered temperatures ranging from 141 to $342 \mathrm{~K}$ $\left(-206\right.$ to $\left.156^{\circ} \mathrm{F}\right)$ and pressures up to $35 \mathrm{MPa}(5000 \mathrm{psi})$. [Results are tabulated in Tables 9 through 19 and Tables 17 through 19, DOE/CE/23810-16.

\section{Thermal Conductivity}

The thermal conductivity of HFC-32 was measured at 1030 points covering temperatures from 160 to $340 \mathrm{~K}\left(-167\right.$ to $\left.160^{\circ} \mathrm{F}\right)$ and pressures up to $70 \mathrm{MPa}(10,000 \mathrm{psi})$. [Results are presented in Table 22, DOE/CE/23810-16]. Figure 19-1 is a plot of the thermal conductivity surface.

\section{Shear Viscosities}

Shear viscosities of compressed and saturated fluid HFC-32 were measured using two torsionally oscillating, quartz-crystal viscometers. [Results are presented in Tables 24 and 25 , DOE/CE/23810-16. NIST Correlated the data to the following equation:

$$
\begin{aligned}
& \eta^{-1}=406.1(\mathrm{~V}-0.0340) \\
& \text { where } \eta \text { is viscosity in mPa-s } \\
& \quad \mathrm{V} \text { is the molar volume in mol/L }
\end{aligned}
$$

\section{$\underline{\mathrm{HCFC}-123}$}

\section{MBWR Equation of State}

NIST has revised its MBWR equation of state and its ideal gas heat capacity $\left(\mathrm{C}_{\mathrm{p}}^{\circ}\right)$ equation for HCFC-123 (see Table 19-2). This work was prompted by an evaluation of the equations of state for HFC-134a and HCFC-123 carried out by Annex 10 of the International Energy Agency. Weaknesses revealed during the evaluation included the derived properties for speed of sound and heat capacity. The revised equation is reported to be valid at temperatures from just above the triple point up to $550 \mathrm{~K}\left(530^{\circ} \mathrm{F}\right)$ and at pressures up to $40 \mathrm{MPa}(5800 \mathrm{psi})$.

\section{Thermal Conductivity}

The thermal conductivity of HCFC-123 was measured at 11618 points. Liquid-phase data cover temperatures from 180 to $440 \mathrm{~K}\left(-136\right.$ to $\left.332^{\circ} \mathrm{F}\right)$ and pressures up to $70 \mathrm{MPa}$ $(10,000 \mathrm{psi})$. Vapor-phase data cover temperaturès from 290 to $449 \mathrm{~K}\left(62\right.$ to $\left.332^{\circ} \mathrm{K}\right)$. [Results are presented in Table 75, DOE/CE/23810-16. Figure 19-2 is a plot of the thermal conductivity surface. 


\section{Molar Heat Capacity}

NIST measured the molar heat capacity of HCFC-123 using an adiabatic calorimeter. Measurements included 79 values in the single phase liquid state and 92 values in the saturated-liquid state. The measurements covered temperatures ranging from 167 to 341 $\mathrm{K}\left(-159\right.$ to $\left.155^{\circ} \mathrm{F}\right)$ and pressures up to $35 \mathrm{MPa}(5000 \mathrm{psi})$. [Results are tabulated in Tables 66 through 71 and Tables 72 through 73, DOE/CE/23810-16].

\section{HCFC-124}

MBWR Equation of State

NIST has revised its 32-term MBWR equation of state and its ideal gas heat capacity $\left(\mathrm{C}_{\mathrm{p}}^{\circ}\right.$ ) equation for HCFC-124 (see Table 19-3). The equation is reported to be valid at temperatures ranging from 210 to $450 \mathrm{~K}\left(-82\right.$ to $\left.350^{\circ} \mathrm{F}\right)$ and it may be reasonably extrapolated up to $500 \mathrm{~K}\left(440^{\circ} \mathrm{F}\right)$. The maximum pressure for the equation is $20 \mathrm{MPa}$ (3000 psi).

\section{Speed of Sound Measurements}

Speed of sound in HCFC-124 was measured using a cylindrical acoustic resonator along isotherms between 250 and $400 \mathrm{~K}\left(-9\right.$ and $\left.261^{\circ} \mathrm{F}\right)$ at pressures ranging from 20 to $900 \mathrm{kPa}$ (3 to $130 \mathrm{psi}$ ). [Results are presented in Table 30, DOE/CE/23810-16]. NIST analyzed the speed of sound measurements at low pressures to determine the ideal-gas heat capacity, $\mathrm{C}_{\mathrm{p}}{ }^{\circ}$ [Revised results are presented in Table 19-3].

\section{Molar Heat Capacity}

The molar heat capacity of HCFC-124 was measured using an adiabatic calorimeter. Measurements included 74 values in the single phase liquid state and 132 values in the saturated-liquid state. The measurements covered temperatures ranging from 173 to 345 $\mathrm{K}\left(-148\right.$ to $\left.161^{\circ} \mathrm{F}\right)$ and pressures up to $35 \mathrm{MPa}(5000 \mathrm{psi})$. [Results are tabulated in Tables 33 through 37 and Table 38, DOE/CE/23810-16].

\section{$\underline{\text { HFC-125 }}$}

MBWR Equation of State

NIST has revised its 32-term MBWR equation of state and its ideal gas heat capacity $\left(\mathrm{C}_{\mathrm{p}}^{\circ}\right)$ for HFC-125 (see Table 19-4). The equation is reported to be valid at temperatures ranging from 200 to $400 \mathrm{~K}\left(-100\right.$ to $\left.260^{\circ} \mathrm{F}\right)$. It may be reasonably extrapolated up to 500 $\mathrm{K}\left(440^{\circ} \mathrm{F}\right)$. The maximum pressure for the equation is $20 \mathrm{MPa}(2900 \mathrm{psi})$. 
Speed of Sound Measurements

Speed of sound in HFC-125 was measured using a cylindrical acoustic resonator along isotherms between 240 and $380 \mathrm{~K}\left(-27.1\right.$ and $\left.224.3^{\circ} \mathrm{F}\right)$ at pressures up to $1 \mathrm{MPa}(145 \mathrm{psi})$. [Results are presented in Table 46, DOE/CE/23810-16]. NIST analyzed the speed of sound measurements as low pressures to determine the ideal-gas heat capacity, $\mathrm{C}_{\mathrm{p}}{ }^{\circ}[$ Revised .results are presented in Table 19-4]. 
Table 19-1. Coefficients to the MBWR Equation of State for HFC-32. [units are $\mathbf{K}$, bar, L, mol] (fit of Outcalt, 8-2-94)

$$
P=\quad \sum_{n=1}^{9} a_{n} \rho^{n}+\exp \left(-\rho^{2} / \rho_{c}^{2}\right) \sum_{n=10}^{15} a_{n} \rho^{2 n-17}
$$

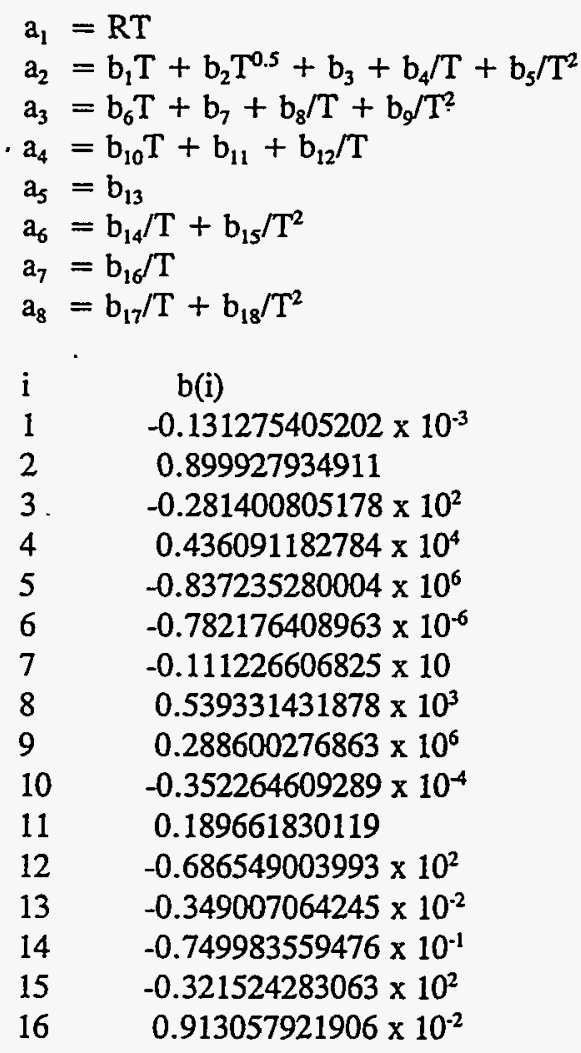

$a_{7}=b_{16} / \mathrm{T}$

$a_{8}=b_{17} / T+b_{18} / T^{2}$

b(i)

$-0.131275405202 \times 10^{-3}$

$a_{9}=b_{19} / T^{2}$

$a_{10}=b_{20} / T^{2}+b_{21} / T^{3}$

$a_{11}=b_{22} / T^{2}+b_{23} / T^{4}$

$a_{12}=b_{24} / \mathrm{T}^{2}+b_{25} / \mathrm{T}^{3}$.

$a_{13}=b_{26} / T^{2}+b_{27} / T^{4}$

$a_{14}=b_{28} / T^{2}+b_{29} / T^{3}$

$a_{15}=b_{30} / T^{2}+b_{31} / T^{3}+b_{32} / T^{4}$

$\begin{array}{lr}\text { i } & \text { b(i) } \\ 17 & -0.17108\end{array}$

$17-0.171082181849 \times 10^{-3}$

$18 \quad 0.503986984347 \times 10^{-1}$

$19-0.830354867752 \times 10^{-3}$

$20 \quad-0.245522676708 \times 10^{6}$

$21-0.107859056038 \times 10^{8}$

$22 \quad-0.429514279646 \times 10^{4}$

$23 \quad 0.808724729567 \times 10^{8}$

$24-0.125945229993 \times 10^{2}$

$25-0.105735009761 \times 10^{4}$

$26-0.904064745354 \times 10^{-1}$

$27-0.183578733048 \times 10^{4}$

$28 \quad-0.169690612464 \times 10^{-3}$

$29 \quad 0.639250820631 \times 10^{-1}$

$30 \quad-0.204925767440 \times 10^{-6}$

$31-0.165629700870 \times 10^{-3}$

$32-0.932607493424 \times 10^{-2}$

critịcal parameters:

$$
\begin{array}{ll}
\mathrm{P}_{\mathrm{c}} & =57.95 \mathrm{bar} \\
\mathrm{P}_{\mathrm{c}} & =8.2078 \mathrm{~mol} / \mathrm{L} \\
\mathrm{T}_{\mathrm{c}} & =351.35 \mathrm{~K} \\
\mathrm{R} & =0.08314471 \mathrm{Lbar} /(\mathrm{mol} \cdot \mathrm{K})
\end{array}
$$

Ideal Gas Heat Capacity Equation [units are $\mathrm{K}$ and $\mathrm{J} /(\mathrm{mol} \cdot \mathrm{K})$ ]

$$
\begin{array}{ll}
\mathrm{C}_{\mathrm{p}}^{\circ}=\mathrm{c}_{0}+\mathrm{c}_{1} \mathrm{~T}+\mathrm{c}_{2} \mathrm{~T}^{2}+\mathrm{c}_{3} \mathrm{~T}^{3} \\
\mathrm{i} & \mathrm{c}(\mathrm{i}) \\
0 & 36.79959 \\
1 & -6.304821 \times 10^{-2} \\
2 & 3.757936 \times 10^{-4} \\
3 & -3.219812 \times 10^{-7}
\end{array}
$$


Table 19-2. Coefficients to the MBWR Equation of State for HCFC-123. (units are K, bar, L, mol) (fit of Younglove 3-25-94)

$$
P=\quad \sum_{n=1}^{9} a_{n} \dot{p}^{n}+\exp \left(-\rho^{2} / \rho_{c}^{2}\right) \sum_{n=10}^{15} a_{n} \rho^{2 n-17}
$$

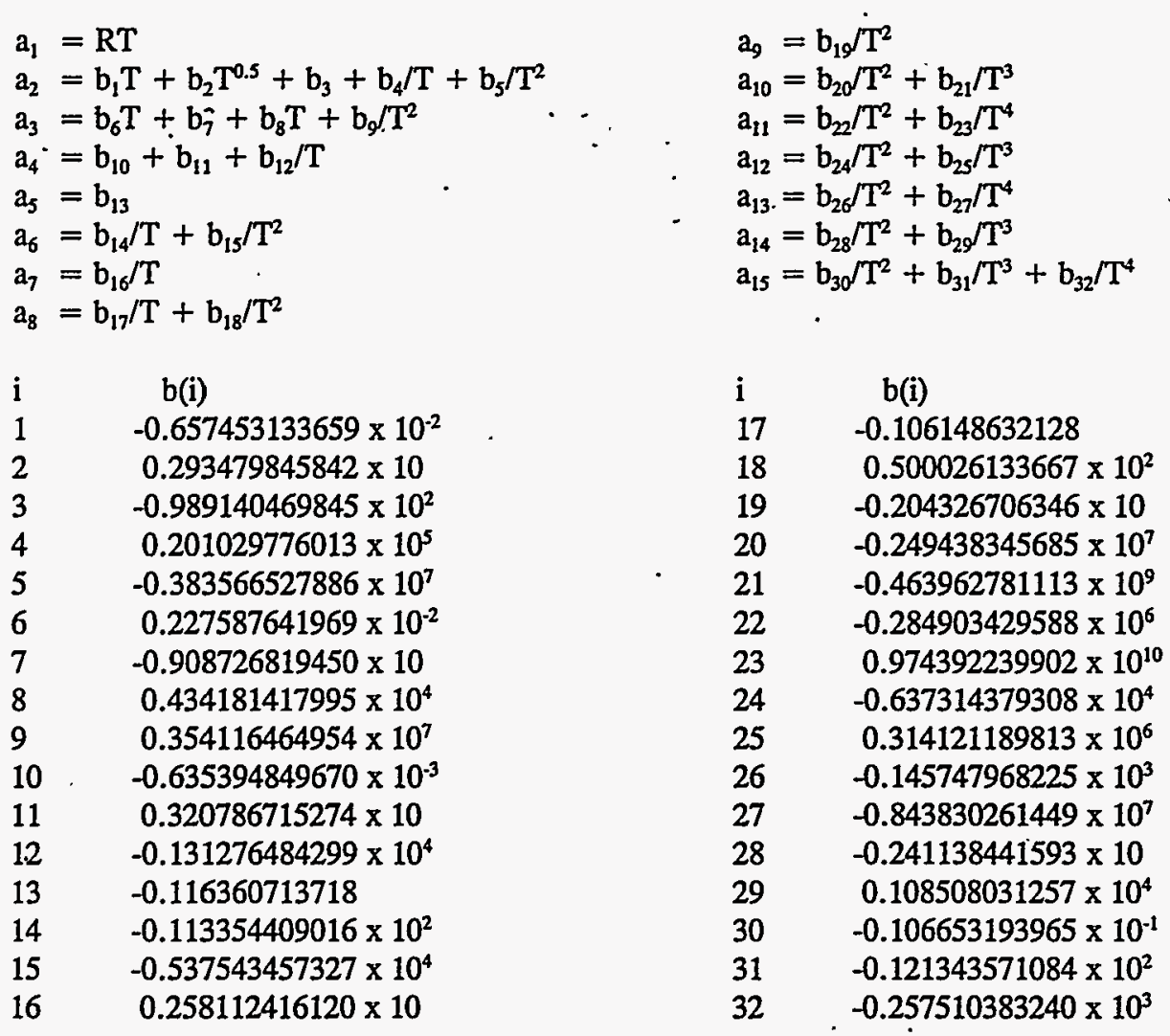

critical parameters:

$$
\begin{array}{ll}
\mathrm{P}_{\mathrm{c}} & =36.618 \mathrm{bar} \\
\mathrm{\rho}_{\mathrm{c}} & =3.596417 \mathrm{~mol} / \mathrm{L} \\
\mathrm{T}_{\mathrm{c}} & =456.831 \mathrm{~K} \\
\mathrm{R} & \quad=0.08314510 \cdot \mathrm{L} \mathrm{bar} /(\mathrm{mol} \cdot \mathrm{K})
\end{array}
$$

Ideal Gas Heat Capacity Equation [units. are $\mathrm{K}$ and $\mathrm{J} /(\mathrm{mol} \cdot \mathrm{K})$ ]

$$
\begin{aligned}
& \mathrm{C}_{\mathrm{p}}^{\circ}=\mathrm{c}_{0}+\mathrm{c}_{1} \mathrm{~T}+\mathrm{c}_{2} \mathrm{~T}^{2}+\mathrm{c}_{3} \mathrm{~T}^{3} \\
& \text { i } \quad \mathrm{c}(\mathrm{i}) \\
& 0 \quad 17.01154 \\
& 10.4046308 \\
& 2 \quad-4.644803 \times 10^{-4} \\
& 3 \quad 2.347418 \times 10^{-7}
\end{aligned}
$$


Table 19-3. Coefficients to the MBWR Equation of State for HCFC-124. (units are K, bar, L, mol) (fit of Younglove 6-9-93)

$$
P=\quad \sum_{n=1}^{9} a_{n} \rho^{n}+\exp \left(-\rho^{2} / \rho_{c}^{2}\right) \sum_{n=10}^{15} a_{n} \rho^{2 n-17}
$$

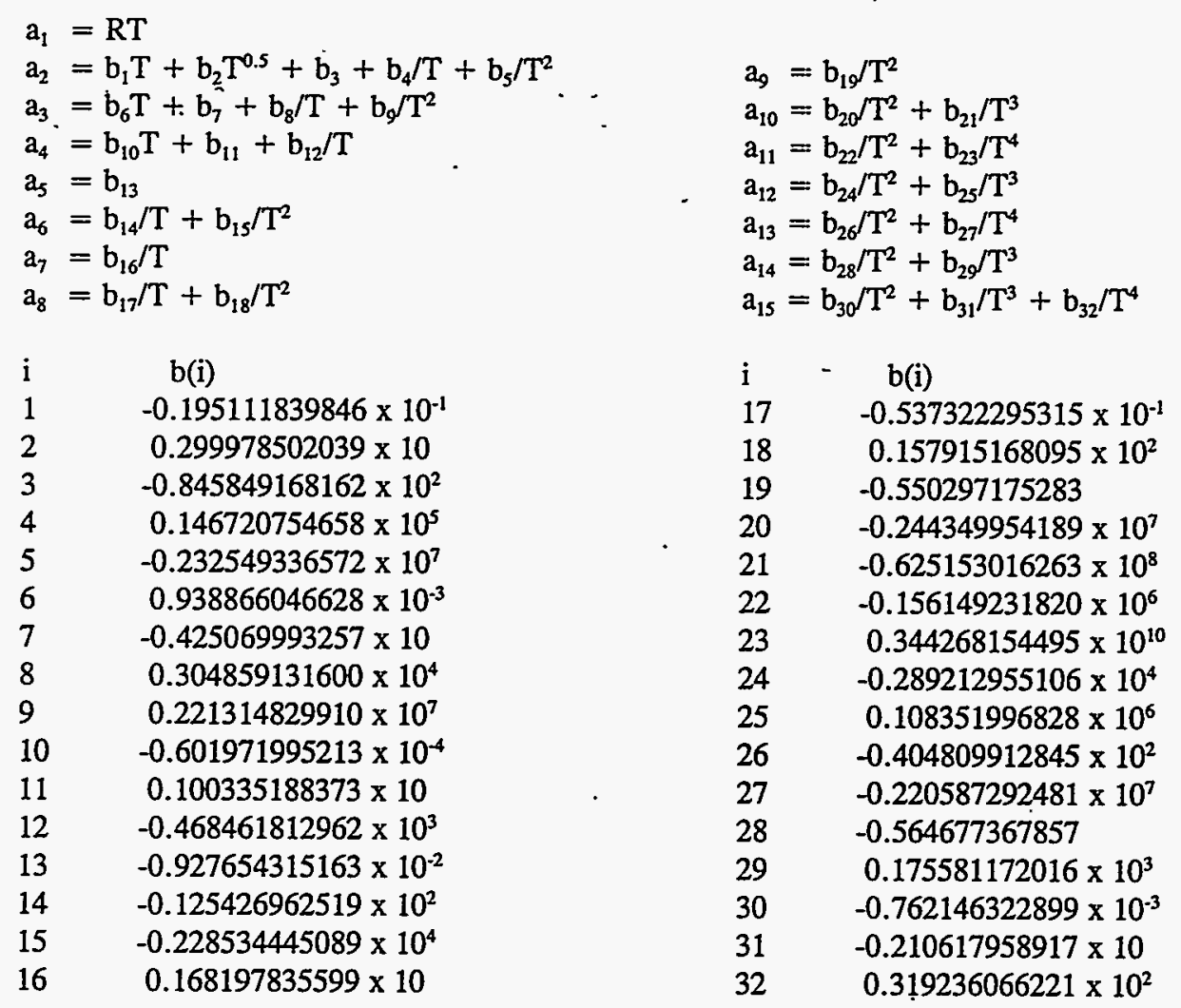

critical parameters:

$$
\begin{array}{ll}
\mathrm{P}_{c} & =36.37 \mathrm{bar} \\
\mathrm{P}_{c} & =4.101527 \mathrm{~mol} / \mathrm{L} \\
\mathrm{T}_{c} & =395.62 \mathrm{~K} \cdot \\
\mathrm{R} & =0.08314510 \mathrm{~L} \cdot \mathrm{bar} /(\mathrm{mol}-\mathrm{K})
\end{array}
$$

Ideal Gas Heat Capacity Equation [units are $\mathrm{K}$ and $\mathrm{J} /(\mathrm{mol} \cdot \mathrm{K})$ ]

$$
\begin{array}{cl}
\mathrm{C}_{p}^{\circ}=c_{0}+c_{1} \mathrm{~T}+\mathrm{c}_{2} \mathrm{~T}^{2}+\mathrm{c}_{3} \mathrm{~T}^{3} \\
\mathrm{i} & \mathrm{c}(\mathrm{i}) \\
0 & 26.65068 \\
1 & 0.2824672 \\
2 & -1.233470 \times 10^{-4} \\
3 & -5.677589 \times 10^{-8}
\end{array}
$$


Table 19-4. Coefficients to the MBWR equation of state for HFC-125 (units are K, bar, L, mol)

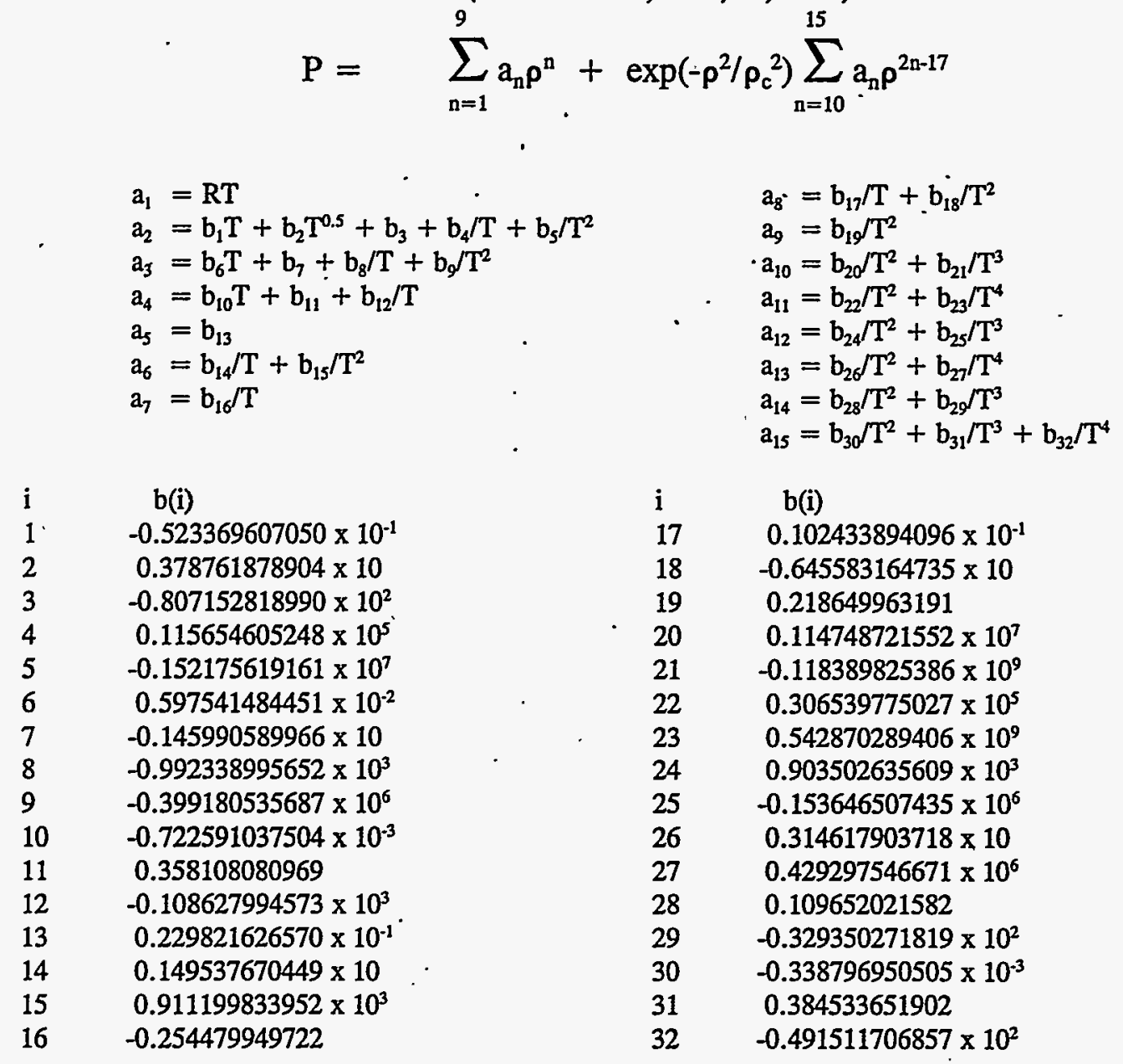

- critical parameters:

$$
\begin{aligned}
\mathrm{P}_{\mathrm{c}} & \doteq 36.29 \mathrm{bar} \\
\cdot \mathrm{P}_{\mathrm{c}} & =4.75996 \mathrm{~mol} / \mathrm{L} \\
\mathrm{T}_{\mathrm{c}} & =339.33 \mathrm{~K} \\
\mathrm{R} & =0.08314471 \mathrm{Lbar} /(\mathrm{mol}-\mathrm{K})
\end{aligned}
$$

Ideal Gas Heat Capacity Equation [units are $\mathrm{K}$ and $\mathrm{J} /(\mathrm{mol} \cdot \mathrm{K})$ ]

$$
\begin{array}{cc}
\mathrm{C}_{\mathrm{p}}^{\circ}=\mathrm{c}_{0}+\mathrm{c}_{1} \mathrm{~T}+\mathrm{c}_{2} \mathrm{~T}^{2}+\mathrm{c}_{3} \mathrm{~T}^{3} \\
\mathrm{i} & \mathrm{c}(\mathrm{i}) \\
0 & 25.87069 \\
1 & 0.2690914 \\
2 & -1.331388 \times 10^{-4} \\
3 & 4.101330 \times 10^{-9} \\
& \\
& . \\
& 19-8
\end{array}
$$


Figure 19-1. Thermal Conductivity Surface of HFC-32

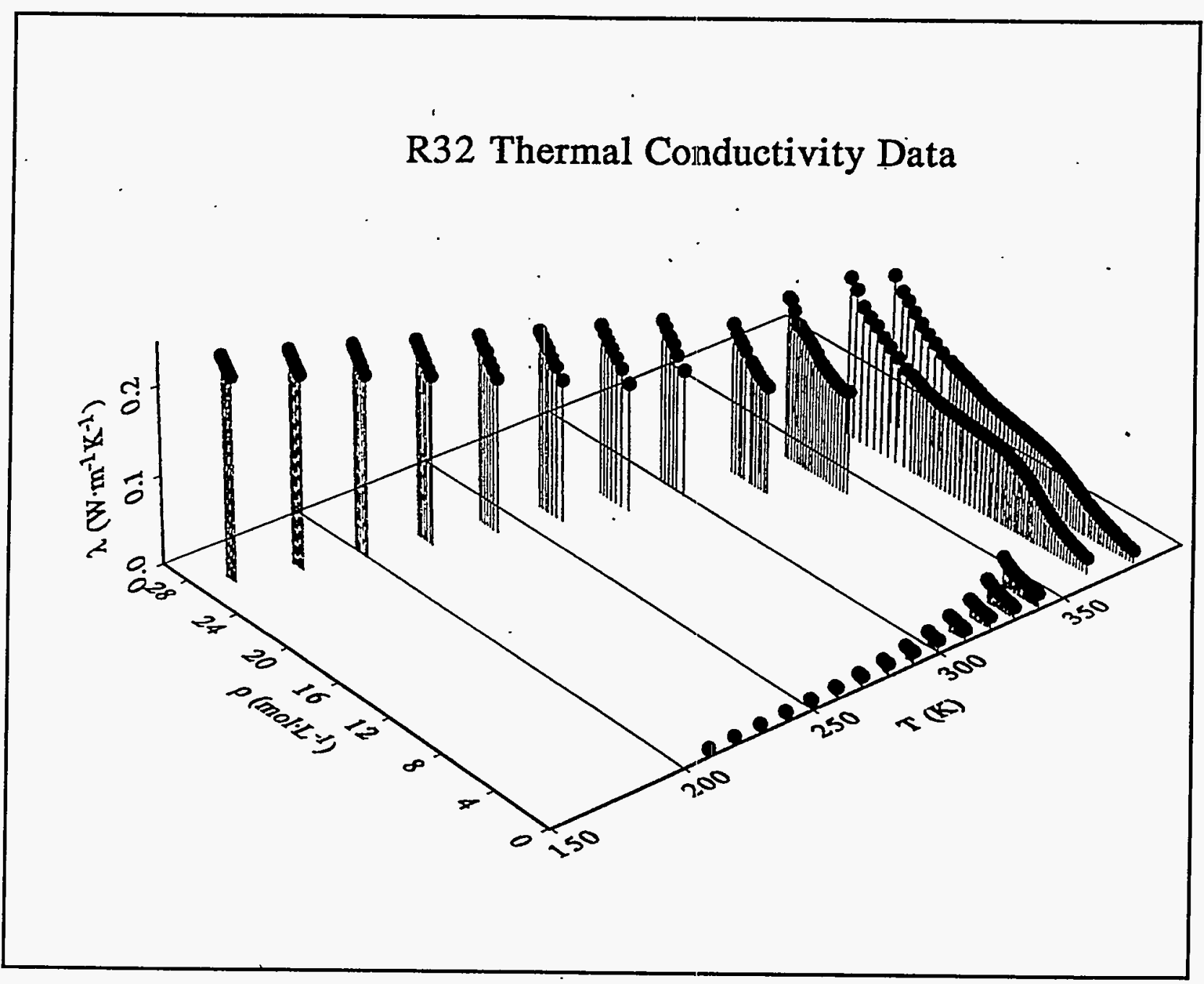


Figure 19-2. Thermal Conductivity Surface of HCFC-123

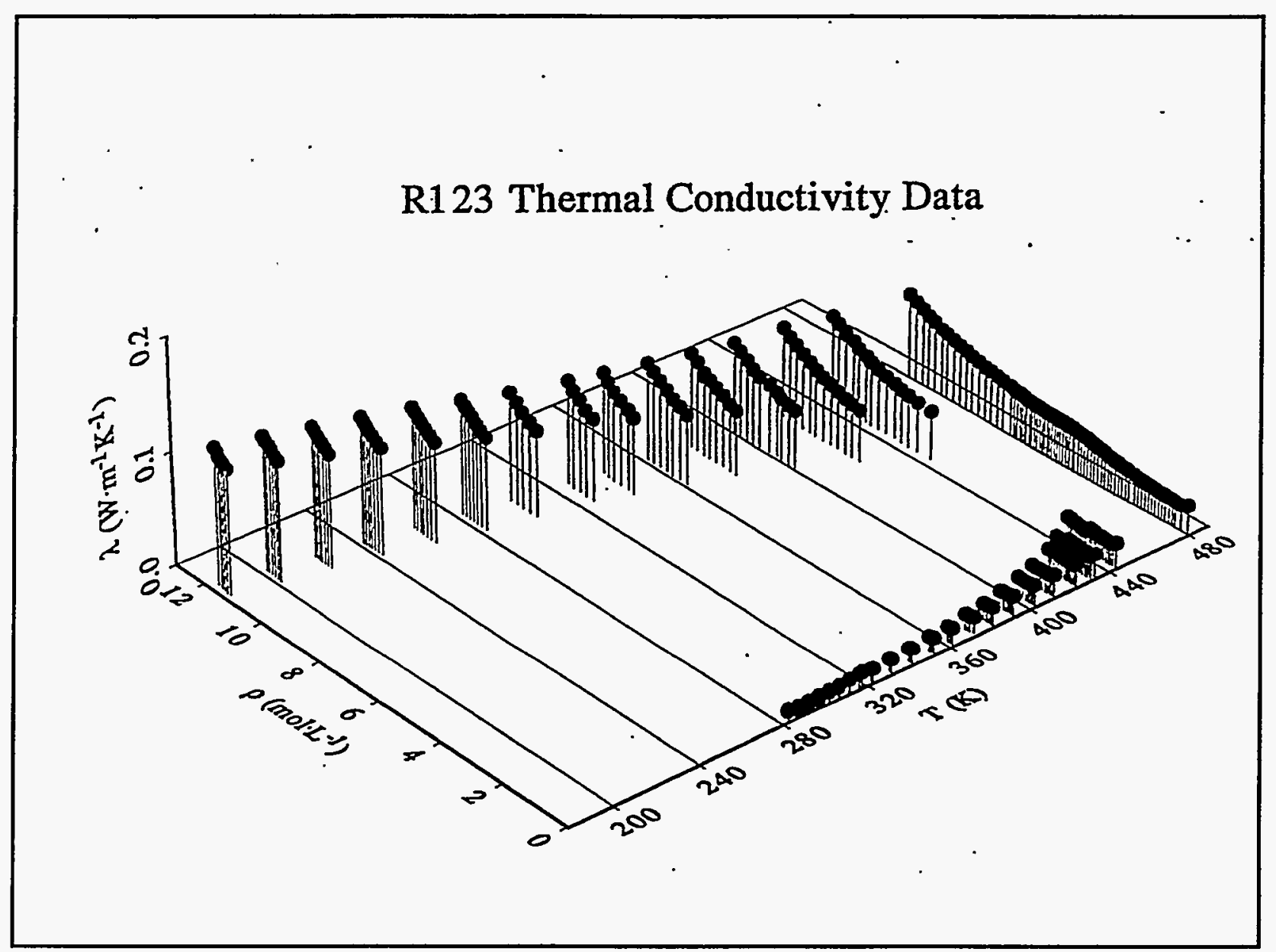




\section{THERMOPHYSICAL PROPERTIES OF \\ HFC-143a AND HFC-152a}

\section{Objective:}

To provide highly accurate, selected thermophysical properties data for refrigerants HFC143a $\left(\mathrm{CH}_{3} \mathrm{CF}_{3}\right)$ and HFC-152a $\left(\mathrm{CH}_{3} \mathrm{CHF}_{2}\right)$; and to fit these data to theoretically-based . equations of state and detailed transport property models.

Results:

The Thermophysics Division of the National Institute of Standards and Technology (NIST) at Boulder, CO, has conducted measurements and correlations of HFC-143a and HFC152a. The new data filled gaps in existing data sets and resolved the problems and uncertainties that existed in and between those data sets. Measurements and determinations of thermodynamic properties included vapor and liquid pressure-volumetemperature (PVT) behavior, saturation and critical points, vapor speed of sound, ideal gas heat capacity, and isochoric heat capacity. The data was then fitted to the modified Benedict-Webb-Rubin (MBWR) equation of state. Measurements and correlations of transport properties included thermal conductivity and viscosity. Results are contained in the final report, DOE/CE-23810-39, Thermophysical Properties of HFC-143a and HFC$152 a$, July 1994 , by $\mathrm{W}$. M. Haynes, $\mathrm{PhD}$. These results are summarized below.

\section{$\underline{\mathrm{HFC}-143 \mathrm{a}}$}

\section{MBWR Equation of State}

NIST has analyzed thermophysical properties measurements from this project and data from existing literature to develop a 32-term modified Benedict-Webb-Rubin equation of state for HFC-143a. Table 20-1 provides the coefficients to the MBWR equation of state. The MBWR equation of state is reported to be valid at temperatures from 180 to $400 \mathrm{~K}$ $\left(-136\right.$ to $260^{\circ} \mathrm{F}$ ) and for pressures up to $40 \mathrm{MPa}$ (5800 psia). The equation may be reasonably extrapolated from the triple point temperature of $162 \mathrm{~K}$ up to $500 \mathrm{~K}(-168$ to $\left.440^{\circ} \mathrm{F}\right)$ and for pressures up to $100 \mathrm{MPa}(14,500 \mathrm{psia})$.

\section{Speed of Sound Measurements}

The speed of sound in HFC-143a was measured using a cylindrical acoustic resonator. Measurements were conducted along isotherms ranging from 235.0 to $400.0 \mathrm{~K}$ (-36.7 to $260.3^{\circ} \mathrm{F}$ ) and at pressures from 40 to $1000 \mathrm{kPa}$ (6 to $\left.145.0 \mathrm{psia}\right)$. [The results are presented in Table 7, DOE/CE/23810-39]. NIST analyzed this data to determine the ideal-gas heat capacity, $\mathrm{C}_{\mathrm{p}}{ }^{\circ}$. [Results are presented in Table 8, DOE/CE-23810]. These data were then fitted to the 
following equation:

$$
\mathrm{C}_{\mathrm{p}}{ }^{\circ}=\mathrm{c}_{0}+\mathrm{c}_{1} \mathrm{~T}+\mathrm{c}_{2} \mathrm{~T}^{2}+\mathrm{c}_{3} \mathrm{~T}^{3}
$$

where:

\section{SI UNITS}

$$
\begin{array}{ll}
c_{0} & =19.09245 \\
c_{1} & =0.2035019 \\
c_{2} & =2.607884 \times 10^{-5} \\
c_{3} & =-1.724083 \times 10^{-7} \\
\mathrm{~T} & =\text { temperature in } \mathrm{K}
\end{array}
$$

\section{Liquid Molar Heat Capacity}

NIST measured the molar heat capacity at constant pressure for HFC-143a. Measurement included 136 values in the liquid state and 84 values in the vapor + liquid two-phase region. The measurements covered temperatures from 165 to $343 \mathrm{~K}\left(-163\right.$ to $\left.158^{\circ} \mathrm{F}\right)$ and pressures up to $35 \mathrm{MPa}$ (5100 psi). [Results are presented in Tables 10 through 13, DOE/CE/23810-39]

\section{Thermal Conductivity Measurements}

The thermal conductivity of HFC-143a was measured at 1229 points (121 points at steady state and 1108 transient measurements). [Results are presented in Tables 18 and 19, DOE/CE/2381039]. The measurements covered temperatures from 191 to $373 \mathrm{~K}\left(-116\right.$ to $\left.212^{\circ} \mathrm{F}\right)$ and pressures up to $70 \mathrm{MPa}(10,200$ psia). Figure 20-1 depicts a plot of the thermal conductive surface for HFC-143a.

\section{Shear Viscosity}

The shear viscosity of compressed vapor and saturated liquid HFC-143a were measured at temperatures from 255.6 to $337.8 \mathrm{~K}\left(0.4\right.$ to $\left.148.4^{\circ} \mathrm{F}\right)$ using a torsionally oscillating quartz crystal viscometer for the vapor measurements and a capillary viscometer for the liquid measurement. [Results are presented in Tables 21 and 22, DOE/CE/23810-39]. NIST correlated the saturated liquid viscosity data to the following equation:

$$
\begin{aligned}
& \eta=3.563 \times 10^{-8} e^{53191 \mathrm{~T}^{2}}\left(\mathrm{~V}-5.1608 \times 10^{-4}\right) \\
& \text { where }
\end{aligned}
$$

$\eta$ is viscosity in Pa.s

$\mathrm{T}$ is temperature in $\mathrm{K}$

$\mathrm{V}$ is the specific volume in $\mathrm{m}^{3} / \mathrm{kg}$ 


\section{$\underline{\text { HFC-152a }}$}

\section{MBWR Equation of State}

NIST has revised the 32-term modified Benedict-Webb-Rubin equation of state for HFC152a This revised equation of state will be incorporated into future version of the REFPROP computer program. Table 20-2 provides the revised coefficients to the equation of state. The equation is valid at temperatures from 155 to $450 \mathrm{~K}\left(-181\right.$ to $\left.350^{\circ} \mathrm{F}\right)$ and pressures up to $40 \mathrm{MPa}$ (5800. psia). The equation may be reasonably extrapolated up to $500 \mathrm{~K}\left(440^{\circ} \mathrm{F}\right)$ and pressures up to $100 \mathrm{MPa}(14500 \mathrm{psia})$.

\section{Speed of Sound Measurements}

NIST measured the speed of sound in HFC-152a using a cylindrical acoustic resonator: Measurements were conducted along isotherms fiom 242.8 to $400.0 \mathrm{~K}\left(-22.7\right.$ to $\left.260.3^{\circ} \mathrm{F}\right)$ and at pressures from 35 to $1030 \mathrm{kPa}$ (5 to $149.4 \mathrm{psia}$ ). [Results are presented in Table 30 , DOE/CE/23810-39]. NIST obtained the ideal-gas heat capacity, $\mathrm{C}_{\mathrm{p}}{ }^{\circ}$, by analyzing this data and fitting it to the following equation:

$$
\begin{aligned}
& C_{p}^{0}=c_{0}+c_{1} T+c_{2} T^{2}+c_{3} T^{3} \\
& \text { where: } \quad \text { SI UNITS } \\
& \begin{array}{ll}
\mathrm{c}_{0} & =27.12550 \\
\mathrm{c}_{1} & =9.220968 \times 10^{-2} \\
\mathrm{c}_{2} & =2.189062 \times 10^{-4} \\
\mathrm{c}_{3} & =-2.514364 \times 10^{-7} \\
\mathrm{~T} & =\text { temperature in } \mathrm{K}
\end{array}
\end{aligned}
$$

\section{Molar Heat Capacity}

The molar heat capacity of HFC-152a was measured with an adiabatic calorimeter. Measurements includes 85 points in the single-phase liquid phase and 70 points in the saturated liquid state. Liquid measurements covered temperatures from 164 to $343 \mathrm{~K}$ (164 to $\left.158^{\circ} \mathrm{F}\right)$ and pressures up to $35 \mathrm{MPa}(5100 \mathrm{psia})$. Saturated liquid measurements covered temperatures from 162 to $315 \mathrm{~K}\left(-167\right.$ to $\left.107^{\circ} \mathrm{F}\right)$. [Results are presented in Tables 33 through 36, DOE/CE/23810-39].

\section{Thermal Conductivity Measurements}

NIST has used high-temperature transient hot-wire thermal conductivity instruments to measure the thermal conductivity of HFC-152a at 1588 points (184 stead-state and 1404 transient hot-wire measurements). [Results are presented in Tables 41 and 42, DOE/CE/23810-39]. Figure 20-2 depicts the thermal conductivity surface for HFC-152a. 


\section{Shear Viscosity}

The shear viscosity of compressed vapor and saturated liquid HFC-152a were measured at temperatures from 254.7 to $330.9 \mathrm{~K}\left(-1.2\right.$ to $\left.136^{\circ} \mathrm{F}\right)$ using a torsionally oscillating quartz crystal viscometer for the vapor measurements and a capillary viscometer for the liquid measurement. [Results are presented in Tables 44 and 45, DOE/CE/23810-39]. The saturated liquid viscosity data has been correlated to the following equation:

$$
\begin{aligned}
& \eta=4.536 \times 10^{-8}\left(\mathrm{~V}-8.2740 \times 10^{-4}\right) \\
& \text { where }
\end{aligned}
$$

$\eta$ is viscosity in $\mathrm{Pa}$.

$\mathrm{T}$ is temperature in $\mathrm{K}$

. $\mathrm{V}$ is the specific volume in $\mathrm{m}^{3} / \mathrm{kg}$ 
Table 20-1. Coefficients to the MBWR Equation of State for HFC-143a [Units are K, bar, L, mol] (fit of Outcalt 4-1-94)

$$
P=\quad \sum_{n=1}^{9} a_{n} \rho^{n}+\exp \left(-\rho^{2} / \rho_{c}^{2}\right) \sum_{n=10}^{15} a_{n} \rho^{2 n-17}
$$

$$
\begin{aligned}
& \mathrm{a}_{1}=\mathrm{RT} \\
& \mathrm{a}_{2}=\mathrm{b}_{1} \mathrm{~T}+\mathrm{b}_{2} \mathrm{~T}^{0.5}+\mathrm{b}_{3}+\mathrm{b}_{4} / \mathrm{T}+\mathrm{b}_{5} / \mathrm{T}^{2} \\
& \mathrm{a}_{3}=\mathrm{b}_{6} \mathrm{~T}+\mathrm{b}_{7}+\mathrm{b}_{8} / \mathrm{T}+\mathrm{b}_{9} / \mathrm{T}^{2} \\
& \mathrm{a}_{4}=\mathrm{b}_{10} \mathrm{~T}+\mathrm{b}_{11}+\mathrm{b}_{12} / \mathrm{T} \\
& \mathrm{a}_{5}=\mathrm{b}_{13} \\
& \mathrm{a}_{6}=\mathrm{b}_{14} / \mathrm{T}+\mathrm{b}_{15} / \mathrm{T}^{2} \\
& \mathrm{a}_{7}=\mathrm{b}_{16} / \mathrm{T} \\
& \mathrm{a}_{8}=\mathrm{b}_{17} / \mathrm{T}+\mathrm{b}_{18} / \mathrm{T}^{2} \\
& \mathrm{a}_{9}=\mathrm{b}_{19} / \mathrm{T}^{2} \\
& \mathrm{a}_{10}=\mathrm{b}_{20} / \mathrm{T}^{2}+\mathrm{b}_{21} / \mathrm{T}^{3} \\
& \mathrm{a}_{11}=\mathrm{b}_{22} / \mathrm{T}^{2}+\mathrm{b}_{23} / \mathrm{T}^{4} \\
& \mathrm{a}_{12}=\mathrm{b}_{24} / \mathrm{T}^{2}+\mathrm{b}_{25} / \mathrm{T}^{3} \\
& \mathrm{a}_{13}=\mathrm{b}_{26} / \mathrm{T}^{2}+\mathrm{b}_{27} / \mathrm{T}^{4} \\
& \mathrm{a}_{14}=\mathrm{b}_{28} / \mathrm{T}^{2}+\mathrm{b}_{29} / \mathrm{T}^{3} \\
& \mathrm{a}_{15}=\mathrm{b}_{30} / \mathrm{T}^{2}+\mathrm{b}_{31} / \mathrm{T}^{3}+\mathrm{b}_{32} / \mathrm{T}^{4}
\end{aligned}
$$

$b_{i}$

$0.326053658322 \times 10^{-1}$

$-0.846331139371 \times 10^{-1}$

$-0.305253599792 \times 10^{2}$

$0.917478595120 \times 10^{4}$

$-0.165632008187 \times 10^{7}$

$-0.474205931664 \times 10^{-2}$

$0.568175751594 \times 10$

$-0.232029232656 \times 10^{4}$

$0.728436638001 \times 10^{6}$

$0.214685469778 \times 10^{-3}$

$$
0.132142017636 \times 10^{-1}
$$

$-0.421876231759 \times 10^{2}$

$-0.128899645225 \times 10^{-1}$.

$0.115735615336 \times 10$

$-0.483926814735 \times 10^{3}$

$-0.222296460032 \times 10^{-1}$ $\rho_{\mathrm{c}}=5.14868 \mathrm{~mol} / \mathrm{L}$

$\mathrm{T}_{\mathrm{c}}=346.751 \mathrm{~K}$

$\mathrm{P}_{\mathrm{c}}=38.32$ bar

$\mathrm{R}=8.314510 \mathrm{~L} \cdot \mathrm{bar} /(\mathrm{mol} \cdot \mathrm{K})$ i

$17-0.927939144228 \times 10^{-3}$

$18 \quad 0.250947031242 \times 10$

$19-0.755054824294 \times 10^{-1}$

$20 \quad-0.171719132604 \times 10^{6}$

$21-0.404322973367 \times 10^{8}$

$22-0.119371454920 \times 10^{5}$

$23 \quad 0.238466476268 \times 10^{9}$

$24-0.819911376240 \times 10^{2}$

$25-0.686895987123 \times 10^{4}$

$.26-0.134398312504 \times 10$

$27-0.107791878226 \times 10^{6}$

$28-0.161289900259 \times 10^{-1}$

$29 \quad 0.705806081763 \times 10$

$30 \quad 0.942860255089 \times 10^{-5}$

$31-0.562324749115 \times 10^{-1}$

$32 \quad 0.499692107366 \times 10$ 
Figure 20-1. Thermal Conductivity Surface for HFC-143a.

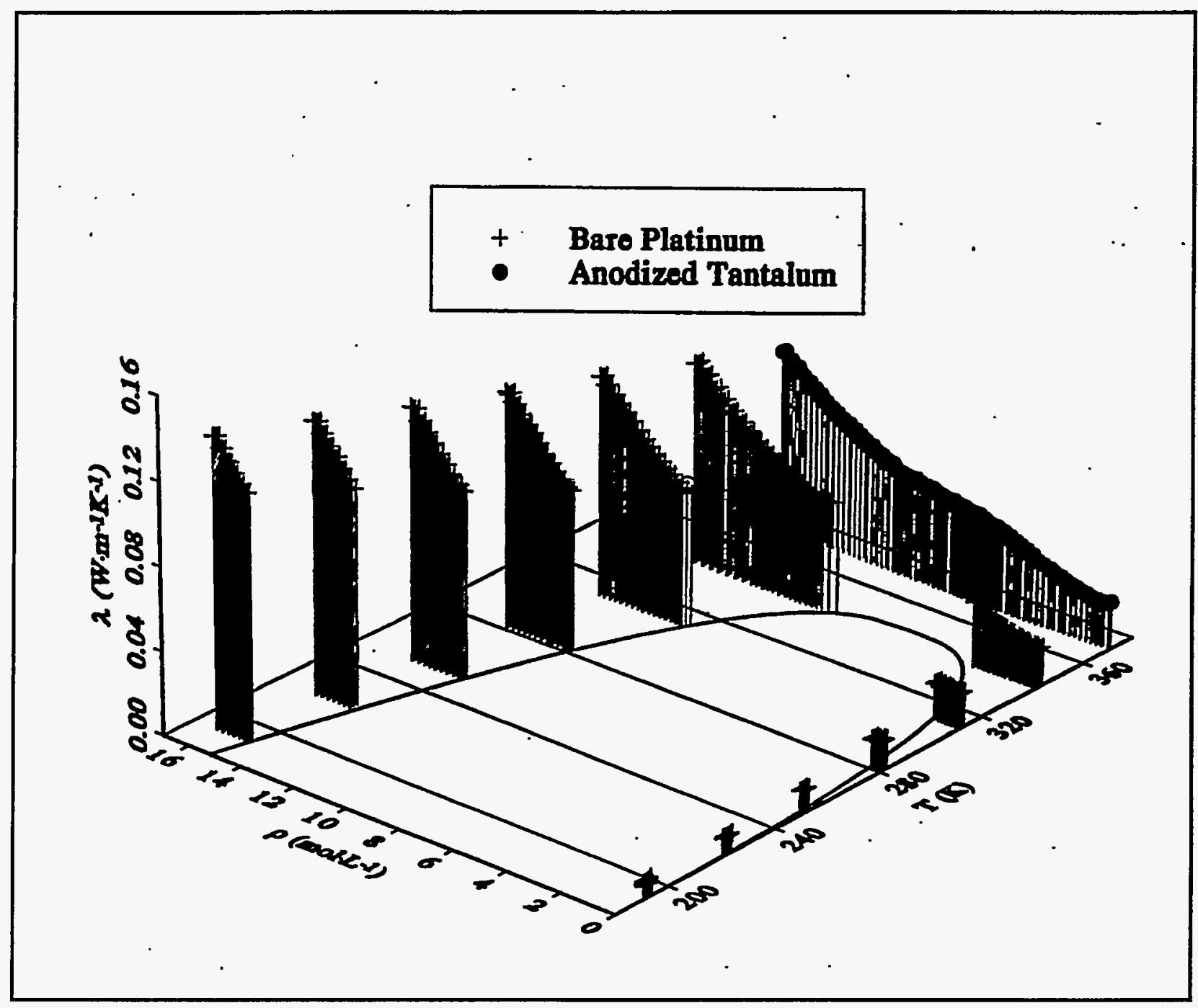


Table 20-2. Revised Coefficients to the MBWR Equation of State for HFC-152a.

(units are K, bar, L, mol) (fit of Outcalt 7-13-94)

$$
P=\quad \sum_{n=1}^{9} a_{n} \rho^{n}+\exp \left(-\rho^{2} / \rho_{c}^{2}\right) \sum_{n=10}^{15} a_{n} \rho^{2 n-17}
$$

$$
\begin{aligned}
& \mathrm{a}_{1}=\mathrm{RT} \\
& \mathrm{a}_{2}=\mathrm{b}_{1} \mathrm{~T}+\mathrm{b}_{2} \mathrm{~T}^{0.5}+\mathrm{b}_{3}+\mathrm{b}_{4} / \mathrm{T}+\mathrm{b}_{5} / \mathrm{T}^{2} \text {. } \\
& \mathrm{a}_{3}=\mathrm{b}_{6} \mathrm{~T}+\mathrm{b}_{7}+\mathrm{b}_{8} / \mathrm{T}+\mathrm{b}_{9} / \mathrm{T}^{2} \\
& a_{4}=b_{10} T+b_{11}+b_{12} / T \\
& a_{5}=b_{13} \\
& a_{6}=b_{14} / T+b_{15} / T^{2} \\
& \mathrm{a}_{7}=\mathrm{b}_{16} / \mathrm{T} \\
& \mathrm{a}_{8 .}=\mathrm{b}_{17} / \mathrm{T}+\mathrm{b}_{18} / \mathrm{T}^{2} \\
& a_{9}=b_{19} / T^{2} \\
& a_{10}=b_{20} / T^{2}+b_{21} / T^{3} \\
& a_{11}=b_{22} / T^{2}+b_{23} / T^{4} \\
& a_{12}=b_{24} / T^{2}+b_{25} / T^{3} \\
& a_{13}=b_{26} / T^{2}+b_{27} / T^{4} \\
& \mathrm{a}_{14}=\mathrm{b}_{28} / \mathrm{T}^{2}+\mathrm{b}_{29} / \mathrm{T}^{3} \\
& a_{15}=b_{30} / T^{2}+b_{31} / T^{3}+b_{32} / T^{4} \\
& \text { i } \quad b_{i} \\
& 1-0.250029315106 \times 10^{-1} \\
& 2 \quad 0.314406758955 \times 10 \\
& 3-0.842501194121 \times 10^{2} \\
& 4 \quad 0.152109896841 \times 10^{5} \\
& 5 \quad-0.235150953572 \times 10^{7} \\
& 6-0.560606848017 \times 10^{-3} \\
& 7 \quad-0.561725012842 \\
& 8 \quad 0.349883524824 \times 10^{3} \\
& 9 \quad 0.671534833264 \times 10^{6} \\
& 10 \quad-0.101677799337 \times 10^{-3} \\
& 11 \quad 0.503738839118 \\
& 12-0.205514094728 \times 10^{3} \\
& 13-0.137760294518 \times 10^{-1} \\
& 14-0.205012592095 \\
& 15-0.220865713923 \times 10^{3} \\
& 16 \quad 0.691474699057 \times 10^{-1}
\end{aligned}
$$

$$
\begin{aligned}
& \dot{\rho_{c}}=5.57145 \mathrm{~mol} / \mathrm{L} \\
& \mathrm{T}_{\mathrm{c}}=386.4 \dot{4} 1 \mathrm{~K} \\
& \mathrm{P}_{\mathrm{c}}=45.167 \mathrm{bar} \\
& \mathrm{R}=0.08314471 \mathrm{Lbar} /(\mathrm{mol} \mathrm{K})
\end{aligned}
$$$$
\text { i } \quad b_{i}
$$

$$
0.758342353876
$$$$
-0.185756493708 \times 10^{-1}
$$$$
-0.437568865038 \times 10^{6}
$$$$
-0.386718918565 \times 10^{8}
$$$$
-0.176762932975 \times 10^{5}
$$$$
0.519483578337 \times 10^{9}
$$$$
-0.160087962199 \times 10^{3}
$$$$
0.773474059810 \times 10^{4}
$$$$
-0.145595794648 \times 10
$$$$
-0.743051998138 \times 10^{5}
$$$$
-0.951744381887 \times 10^{-2}
$$$$
0.387877679400 \times 10
$$$$
-0.195015377121 \times 10^{-4}
$$$$
-0.160761476257 \times 10^{-1}
$$

$-0.841063960548$ 
Figure 20-2. Thermal Conductivity Surface of HFC-152a.

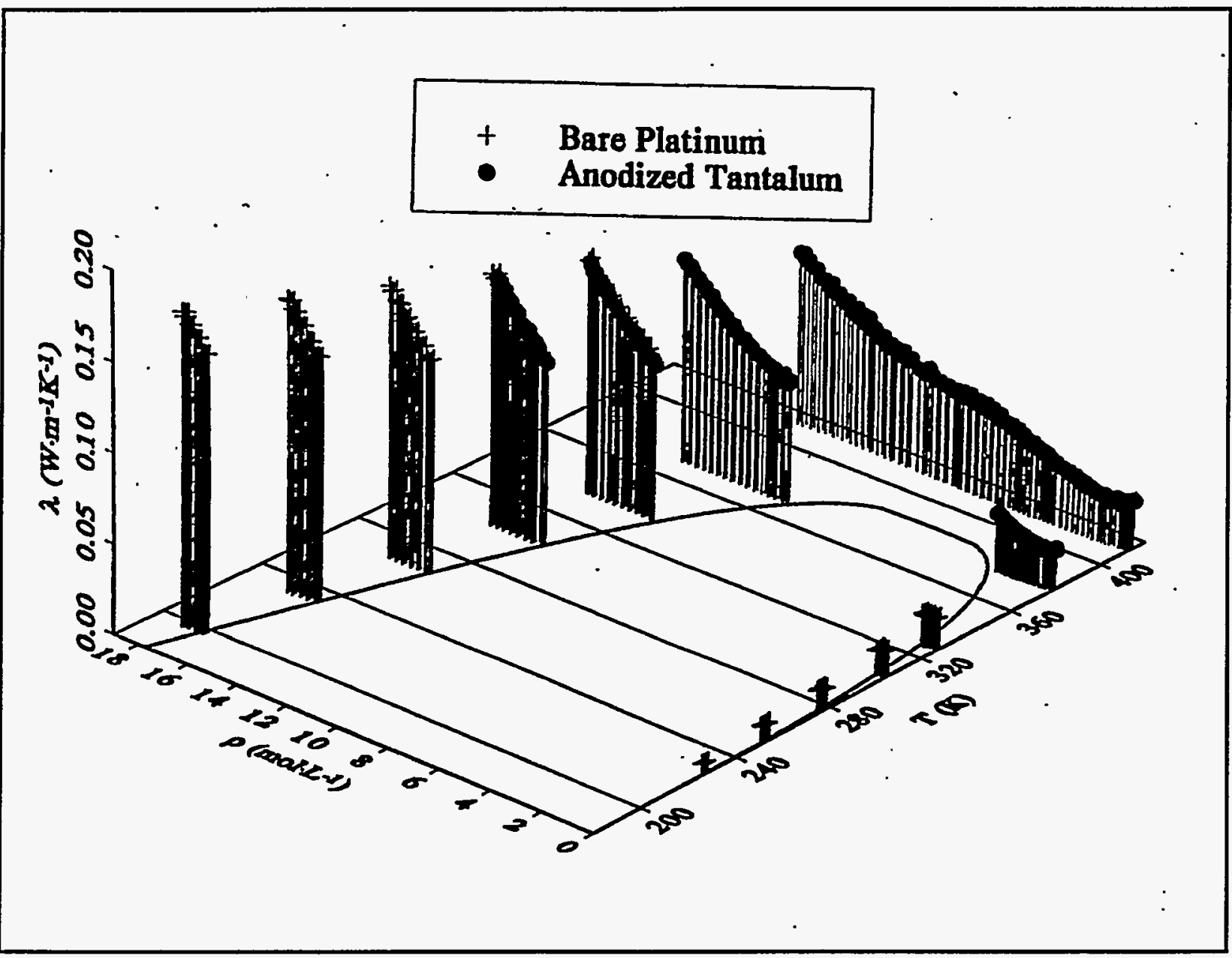


Objective:

To provide information regarding the coefficients of performance (COP), capacities, compressor discharge temperatures, compressor discharge pressures, and compressor discharge pressure ratios of nine alternative fluids relative to $\mathrm{HCFC}-22$ and three alternative fluids relative to $\mathrm{R}-502$.

Results:

The Building Environment Division of the National Institute of Standards and Technology (NIST) completed this research under contract with ARTI. Detailed results of this study are reported in the final report, DOE/CE/23810-7, Theoretical Evaluations of $R-22$ Alternative Fluids, January 1993, by Piotr A. Domanski, $\mathrm{PhD}$ and David A. Didion, $\mathrm{PhD}$. This report is currently available from the ARTI Refrigerant Database (RDB \#3305, 32 pages). The following refrigerants and refrigerant blends were evaluated:

Alternative Refrigerants/Blends (\% Weight)

HCFC-22 Alternatives

HFC-32/HFC-125 (60/40)

HFC-32/HFC-134a (25/75)

HFC-32/HFC-134a (30/70)

HFC-32/HFC-125/HFC-134a (10/70/20)

HFC-32/HFC-125/HFC-134a (30/10/60)

HFC-32/HFC-227ea (35/65)

HFC-32/HFC-125/HFC-134a/R-290 (20/55/20/5)

HFC-134a

R-290 (Propane)

R-502 Alternatives

HFC-32/HFC-125/HFC-143a (10/45/45)

HFC-125/HFC-143a/HFC-134a (44/52/4)

HFC-125/HFC-143a (45/55)

Results of the evaluations are presented in Figures 21-1 and 21-2. 
Figure 21-1. Relative COPs and Capacities of HCFC-22 Alternatives.

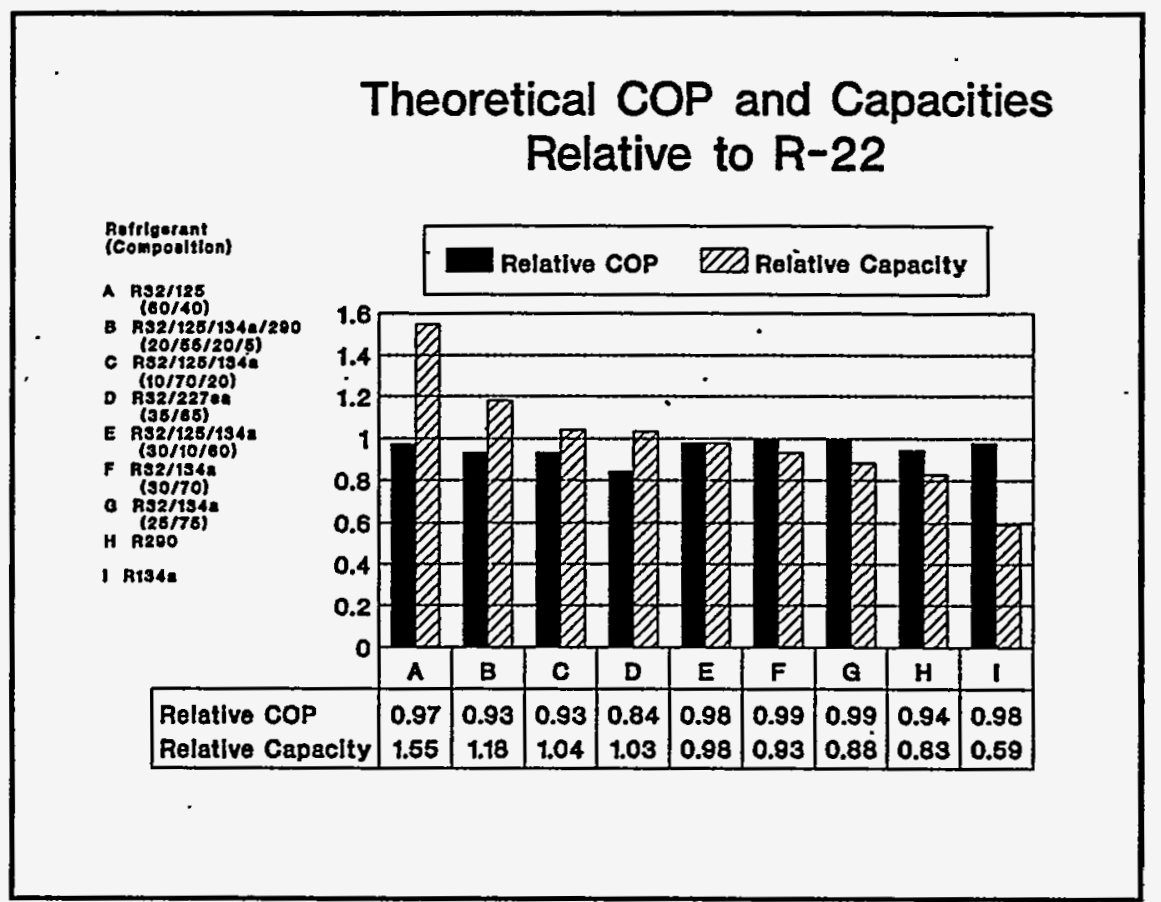

Figure 21-2. Relative COPs and Capacities of R-502 Alternatives.

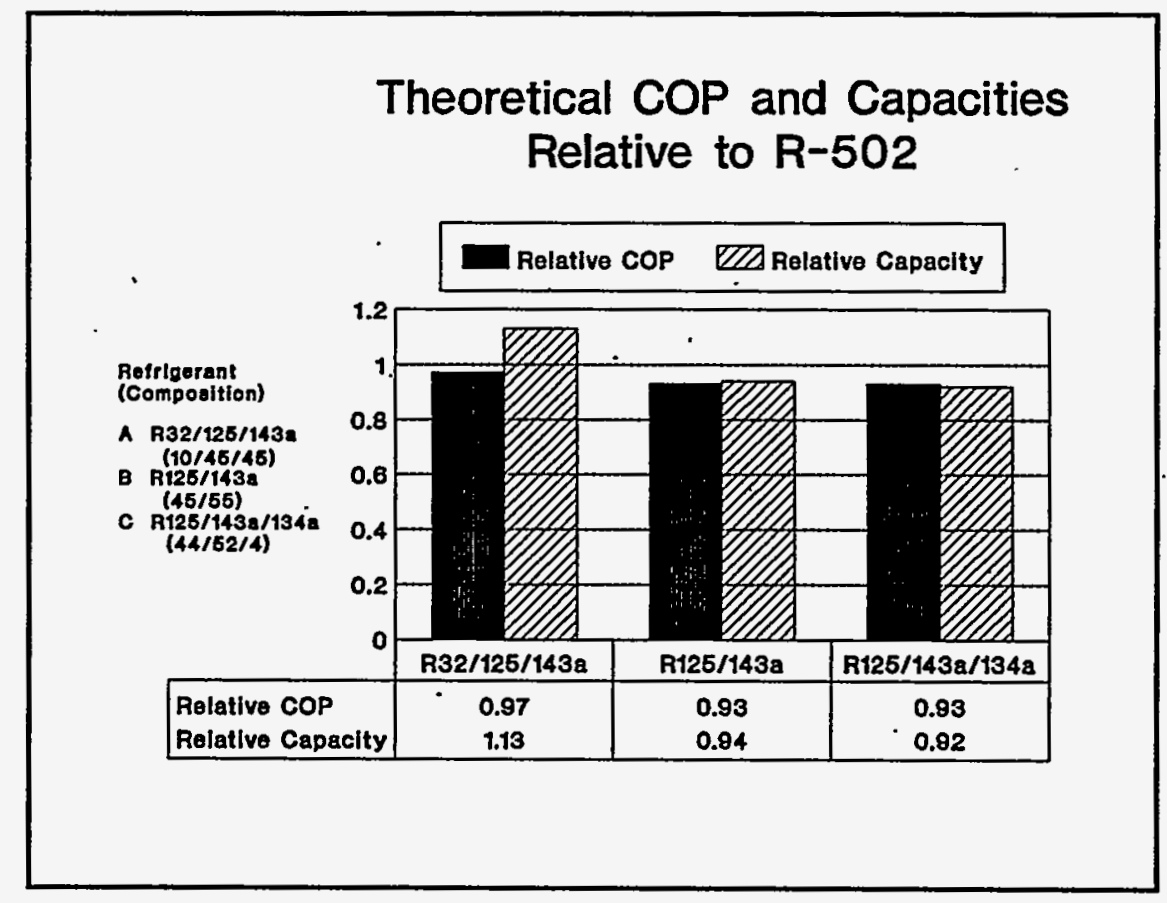

$$
21-2
$$




\section{CHEMICAL AND THERMAL STABILITY \\ OF REFRIGERANT-LUBRICANT MIXTURES WITH METALS}

\section{Objective:}

To provide information on the stability of potential substitutes for CFC refrigerants and appropriate lubricants.

\section{Results:}

Spauschus Associates, Inc., has completed this research under contract with ARTI. A detailed report of results is presented in the final report, DOE/CE/23810-5, Chemical and Thermal Stability of Refrigerant-Lubricant Mixtures with Metals, 9 October 1992, by Dietrich F. Huttenlocher, $\mathrm{PhD}$, (RDB \#3608, 126 pages). Key results are summarized below:

\section{Alternative Refrigerant-Lubricant Combinations}

CFC-11 (baseline) with:

naphthenic mineral oil (ISO 32)

naphthenic mineral oil (ISO 46)

HCFC-22 with:

naphthenic mineral oil (ISO 32)

HFC-32 with:

pentaerythritol ester mixed-acid (ISO 32)

polypropylene glycol butyl monoether (ISO 32)

HCFC-123 with:

naphthenic mineral oil (ISO 32)

naphthenic mineral oil (ISO 46)

HCFC-124 with:

alkylbenzene (ISO 32)

HFC-125 with:

pentaerythritol ester mixed-acid (ISO 32)

polypropylene glycol butyl monoether (ISO 32)

modified polyglycol (ISO 32)

HFC-134 with:

pentaerythritol ester mixed-acid (ISO 32) 


\section{Alternative Refrigerant-Lubricant Combinations (Continued)}

HFC-134a with:

pentaerythritol ester mixed-acid (ISO 22)

pentaerythritol ester branched-acid (ISO 32)

pentaerythritol ester branched-acid (ISO 100)

polypropylene glycol butyl monoether (ISO 32)

polypropylene glycol diol (ISO 22)

modified polyglycol.(ISO 32)

HCFC-142b with:

alkylbenzene (ISO 32)

HFC-143a with:

pentaerythritol ester branched-acid (ISO 32)

HFC-152a with:

alkylbenzene (ISO 32)

Based on the results of his research, Dr. Huttenlocher made the following conclusions:

- All HFCs tested, along with HCFC-22, were very stable and did not undergo any measurable chemical reactions or thermal decompositions at temperatures up to $200^{\circ} \mathrm{C}\left(392^{\circ} \mathrm{F}\right)$.

- HCFC-124 and HCFC-142b were less stable than the HFCs tested but more stable than CFC-12 (a long time industry standard).

- While HCFC-123 was the least stable of the "new" refrigerants tested, it was still ten fold more stable than CFC-11 (the refrigerant it is intended to replace in low pressure chiller applications).

- The pentaerythritol ester lubricants included in the project exhibited acid number increases after aging at $200^{\circ} \mathrm{C}\left(392^{\circ} \mathrm{F}\right)$. The high viscosity (ISO 100) pentaerythritol ester exhibited additional evidence of molecular changes during aging at $200^{\circ} \mathrm{C}$. The formation of $\mathrm{CO}_{2}$ indicated decarboxylation of the high viscosity pentaerythritol ester lubrication at that temperature.

- All of the polyalkylene glycol lubricants had signs of molecular change after aging. 


\section{MISCIBILITY OF LUBRICANTS WITH REFRIGIERANTS}

\section{Objective:}

To provide information on the miscibility of both current and new lubricants with potential substitutes for CFC refrigerants.

\section{Results:}

Iowa State University of Science and Technology is performing this research under contract with ARTI. Phase 1 of the project, preliminary miscibility screening, has been completed. These studies examined mixtures at three refrigerant-lubricant concentrations $(10,50$, and $95 \%$ refrigerant by weight) and a single viscosity for each lubricant. Miscibility studies were conducted over a temperature range of -50 to $90^{\circ} \mathrm{C}\left(-58\right.$ to $\left.194^{\circ} \mathrm{F}\right)$ for most mixtures and -50 to $60^{\circ} \mathrm{C}\left(-58\right.$ to $\left.140^{\circ} \mathrm{F}\right)$ for high pressure refrigerant mixtures. A detailed report on the results of this research is presented in DOE report number DOE/CE/23810-6, Miscibility of Lubricants with Refrigerants (Phase 1), October 1992, by Michael B. Pate, PhD, Steven C. Zoz, and Lyle J. Berkenbosch (RDB \#3503, 64 pages).

Iowa State University has completed Phase 2 of the project which encompassed detailed miscibility plots with five additional refrigerant-lubricant concentrations $(20,35,65,80$ and $90 \%$ refrigerant by weight) and two viscosity grades for each lubricant. The final report, DOE/CE/23810-18, Miscibility of Lubricants with Refrigerants, January 1994, by Michael B. Pate, PhD, Steven C. Zoz, and Lyle J. Berkenbosch, contains detailed results. Preliminary results are summarized in Table 23-1. 
Table 23-1. Miscibility of Lubricants with Refrigerants.

\begin{tabular}{|c|c|c|c|c|c|c|c|c|c|c|}
\hline \multirow[b]{2}{*}{ Lubricant } & \multicolumn{10}{|c|}{ Refrigerant } \\
\hline & R22 & R32 & R123 & R124 & R125 & R134 & R134a & R142b & R143a & R152a \\
\hline $\begin{array}{l}\text { Minaral OII } \\
\text { IsO } 32 \text { cSt }\end{array}$ & $\mid \begin{array}{l}>-100 \\
<36 \% \\
>80 \%\end{array}$ & 1 & M & $\begin{array}{c}>200 \\
08 \\
<23 \%\end{array}$ & I & 1 & I & $\mid \begin{array}{l}>-40 C \\
<50 x \\
>80 x\end{array}$ & 1 & 1 \\
\hline $\begin{array}{l}\text { Minora Oil } \\
\text { ISO } 68 \text { csi }\end{array}$ & $\mid \begin{array}{c}>0 \\
\text { or } \\
<36 \%\end{array}$ & I & $\left|\begin{array}{c}>-400 \\
\text { or } \\
<47 x\end{array}\right|$ & $\left|\begin{array}{c}>800 \\
\text { of } \\
<22 \%\end{array}\right|$ & 1 & I & 1 & $\mid \begin{array}{l}>-30 C \\
<21 \% \\
>89 \%\end{array}$ & 1 & I \\
\hline $\begin{array}{l}\text { Alkylbonzene } \\
\text { ISO } 32 \mathrm{cSi}\end{array}$ & $M$ & 1 & $\boldsymbol{M}$ & $M$ & 1 & 1 & 1 & $M$ & 1 & $>50 c$ \\
\hline $\begin{array}{l}\text { Alkylbenzone } \\
\text { ISO } 68 \mathrm{cSt}\end{array}$ & $M$ & I & $M$ & M & 1 & $1 \cdot$ & 1 & $M$ & 1 & $\begin{array}{l}>50 C \\
\text { of } \\
<20 x\end{array}$ \\
\hline $\begin{array}{l}\text { Polypropylene Ghycol } \\
\text { Butyl Monoether } \\
\text { ISO } 32 \text { cSt }\end{array}$ & $M$ & $<53 \%$ & M & M & $\begin{array}{c}<50 c \\
\text { of } \\
<65 \%\end{array}$ & $\begin{array}{c}>-200 \\
\text { or } \\
<88 x\end{array}$ & $\begin{array}{l}<600 \\
\text { or } \\
<81 \%\end{array}$ & $M$ & $<35 \%$ & M \\
\hline $\begin{array}{l}\text { Polypropylene Glycol } \\
\text { Butyl Monoether } \\
\text { ISO 5B cSt }\end{array}$ & $M$ & $<47 \%$ & $\begin{array}{c}<200 \\
\text { or } \\
>21 x\end{array}$ & $M$ & $\left|\begin{array}{c}100 \\
\text { or } \\
<65 \%\end{array}\right|$ & $M$ & $\begin{array}{l}<500 \\
<64 x\end{array}$ & $M$ & $<38 \%$ & $\begin{array}{l}<80 C \\
<80 x \\
>80 \%\end{array}$ \\
\hline $\begin{array}{l}\text { Polypropylene Glyool } \\
\text { Dloi } \\
\text { ISO } 32 \text { oSt }\end{array}$ & $M$ & $M$ & M & M & $M$ & $M$ & $M$ & $M$ & $<34 \%$ & м \\
\hline $\begin{array}{l}\text { Polypropyliene ahyeol } \\
\text { Oiol } \\
\text { iso } 100 \text { cst }\end{array}$ & $M$ & $<49 \%$ & M & $M$ & $\begin{array}{l}<400 \\
\text { or } \\
<80 \%\end{array}$ & $M$ & $\begin{array}{l}<600 \\
01 \\
<68 \%\end{array}$ & $M$ & $<48 x$ & $\begin{array}{l}<700 \\
<81 \% \\
>80 \%\end{array}$ \\
\hline $\begin{array}{l}\text { Modiliod Polyglyeol } \\
\text { iso } 32 \mathrm{cst}\end{array}$ & $\left|\begin{array}{l}>-20 c \\
<23 \% \\
>50 x\end{array}\right|$ & $\begin{array}{l}<600 \\
>100 \\
<21 x\end{array}$ & $\mid>-400$ & \begin{tabular}{|l|}
$>-100$ \\
$<37 \%$ \\
$>81 x$
\end{tabular} & $\begin{array}{l}<300 \\
>100 \\
<20 \% \\
\end{array}$ & $\begin{array}{l}>00 \\
<23 x \\
>79 x\end{array}$ & $\begin{array}{l}>00 \\
<22 x \\
>52 x\end{array}$ & \begin{tabular}{|l|}
$>-40 C$ \\
$<23 x$ \\
$>68 x$
\end{tabular} & 1 & M \\
\hline $\begin{array}{l}\text { Ponteorythithol Estor } \\
\text { mixed acid } \\
\text { ISO 22 cSt }\end{array}$ & $M$ & $\begin{array}{l}<500 \\
>100 \\
<35 \%\end{array}$ & $\mathbf{M}$ & M & M & M & $\begin{array}{l}>-50 C \\
<69 \% \\
>91 \%\end{array}$ & $\mathbf{M}$ & $<38 \%$ & $M$ \\
\hline $\begin{array}{l}\text { Pentanerythithol Estor } \\
\text { mixod acid } \\
\text { IsO } 32 \mathrm{cBt}\end{array}$ & $M$ & \begin{tabular}{|}
$>-200$ \\
$0 t$ \\
$<51 \%$
\end{tabular} \mid & $M$ & M & M & $M$ & 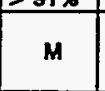 & $M$ & $<49 \times$ & M \\
\hline $\begin{array}{l}\text { Pontaoryth nthol Eetor } \\
\text { mlxod acid } \\
\text { ISO } 100 \mathrm{eSt}\end{array}$ & $M$ & $<35 \%$ & M & M & $\begin{array}{c}<60 C \\
\text { or } \\
<68 \%\end{array}$ & $M$ & $\begin{array}{c}>-100 \\
08 \\
<64 x\end{array}$ & M & $<38 \%$ & M \\
\hline $\begin{array}{l}\text { Pontaerythithol Eator } \\
\text { brenched acid } \\
\text { ISO } 32 \mathrm{cSt}\end{array}$ & $M$ & $>-20$ & $M$ & $M$ & M & $M$ & $M$ & $M$ & $<51 \%$ & $M$ \\
\hline $\begin{array}{l}\text { Pontanythrthol Eator } \\
\text { branched ecld } \\
\text { ISO } 100 \mathrm{cSt}\end{array}$ & M & $<51 x$ & M & $M$ & $\begin{array}{l}<40 C \\
\text { or } \\
<77 x\end{array}$ & $M$ & $\begin{array}{c}<600 \\
\text { or } \\
<70 x\end{array}$ & $M$ & $<34 x$ & $\begin{array}{c}<900 \\
\text { of } \\
<90 x\end{array}$ \\
\hline
\end{tabular}

1 - Immiselbie or miselble orily in a small temperature - concentration region.

$M$ - Mitclble at alf test tomperatures and concentretbons.

<"* - Miscible at all test temporatures or refigorant mass concentretions bolow temperature or concentration indicatod.

$>$ ** - Miscibto at all test tomperatures or refrigerant mase concentrations above temporature or concentration indicatod. 


\section{VISCOSITY, SOLUBILITY AND DENSITY MEASUUREMENTS \\ OF REFRIGERANT-LUBRICANT MIXTURES}

\section{Objective:}

To measure the viscosity, solubility, and density of alternative refrigerant-lubricant mixtures

\section{Results:}

Spauschus Associates, Inc., is performing this research under contract with ARTI. A detailed report of result is contained in the final report, DOE/CE/23810-34, Solubility, Viscosity and Density of Refrigerant/Lubricant Mixtures, by David R. Henderson, PE.

This research involves viscosity, solubility, and density measurements of thirty-five refrigerant-lubricant mixtures listed below at seven different concentrations $(0,10,20,30$, 80,90 , and $100 \%$ refrigerant by weight):

Baseline Mixtures:

CFC-12/mineral oil (ISO $32 \mathrm{cSt}$ )

CFC-12/mineral oil (ISO $100 \mathrm{cSt}$ )

HCFC-22/mineral oil (ISO $32 \mathrm{cSt}$ )

Test Mixtures:

HFC-134a/polypropylene glycol butyl monoether (ISO $68 \mathrm{cSt}$ )

HFC-134a/pentaerythritol ester - mixed acid (ISO $22 \mathrm{cSt}$ )

HFC-134a/pentaerythritol ester - mixed acid (ISO $32 \mathrm{cSt}$ )

HFC-134.a/pentaerythritol ester - mixed acid (ISO $68 \mathrm{cSt}$ )

HFC-134a/pentaerythritol ester - mixed acid (ISO $100 \mathrm{cSt}$ )

HFC-134a/pentaerythritol ester - branched acid (ISO $22 \mathrm{cSt}$ )

HFC-134a/pentaerythritol ester - branched acid (ISO $32 \mathrm{cSt}$ )

HFC-134a/pentaerythritol ester - branched acid (ISO $68 \mathrm{cSt}$ )

HFC-134a/pentaerythritol ester - branched acid (ISO $100 \mathrm{cSt}$ )

HCFC-123/mineral oil (ISO $32 \mathrm{cSt}$ )

HCFC-123/mineral oil (ISO $100 \mathrm{cSt}$ )

HCFC-123/alkylbenzene (ISO $32 \mathrm{cSt}$ )

HCFC-123/alkylbenzene (ISO $68 \mathrm{cSt}$ ) 
Test Mixtures (Continued):

HFC-32/pentaerythritol ester - mixed acid (ISO $22 \mathrm{cSt}$ )

HFC-32/pentaerythritol ester - mixed acid (ISO $68 \mathrm{cSt}$ )

HFC-32/pentaerythritol ester - branched acid (ISO $32 \mathrm{cSt}$ )

HFC-32/pentaerythritol ester - branched acid (ISO $100 \mathrm{cSt}$ )

HFC-125/pentaerythritol ester - mixed acid (ISO $22 \mathrm{cSt}$ )

HFC-125/pentaerythritol ester - mixed acid (ISO $68 \mathrm{cSt}$ )

HFC-125/pentaerythritol ester - branched acid (ISO $32 \mathrm{cSt}$ )

HFC-125/pentaerythritol ester - branched acid (ISO $100 \mathrm{cSt}$ )

HFC-152a/alkylbenzene (ISO $32 \mathrm{cSt}$ )

HFC-152a/alkylbenzene (ISO $68 \mathrm{cSt}$ )

HFC-152a/pentaerythritol ester - mixed acid (ISO $22 \mathrm{cSt}$ )

HFC-152a/pentaerythritol ester - mixed acid (ISO $68 \mathrm{cSt}$ )

HFC-143a/pentaerythritol ester - mixed acid (ISO $22 \mathrm{cSt}$ )

HFC-143a/pentaerythritol ester - mixed acid (ISO $68 \mathrm{cSt}$ )

HFC-143a/pentaerythritol ester - branched acid (ISO $32 \mathrm{cSt}$ )

HFC-143a/pentaerythritol ester - branched acid (ISO $100 \mathrm{cSt}$ )

HCFC-124/alkylbenzene (ISO $32 \mathrm{cSt}$ )

HCFC-124/alkylbenzene (ISO $68 \mathrm{cSt}$ )

HCFC-142b/alkylbenzene (ISO $32 \mathrm{cSt}$ )

Mr. Henderson presents experimental data for each refrigerant-lubricant mixture in the form of curve fitted mathematical models and two charts. One chart presents the density as a function of temperature and concentration. The other presents viscosity and solubility as functions of temperature for given concentrations (Daniel chart).

\section{Low Refrigerant Concentrations}

An oscillating piston viscometer was used to measure viscosities at low refrigerant concentrations. For low refrigerant concentrations viscosity, solubility, and density measurements were fitted to the equations (1) through (4):

\section{High Concentration Refrigerants}

The experimental technique used to measure viscosity for the low refrigerant concentration mixtures was unsuitable for measurement of high refrigerant concentration mixtures for a number or reasons. For the high concentration refrigerant mixture viscosity measurements Mr. Henderson used glass capillary viscometers and differential pressure transducers to measure the pressure differences between a reference bomb containing the $100 \%$ concentration (neat) refrigerant and the two other bombs containing the $90 \%$ and $80 \%$ refrigerant concentration mixtures. The viscometers and pressure bombs were 
thermally controlled in a programmable air bath.

For refrigerant-lubricant mixtures containing HFC-125 or HFC-152a; the refrigerant density is close to the lubricant density which results in data that is not modelled well by the many polynomial equations (cross overs occur near the temperatures where the refrigerant and lubricant densities are equal). For these mixtures containing either of these two refrigerants data was fitted to curves for each concentration.

High refrigerant concentration data (other than HFC-125 and HFC-152a) were fitted to equations (5) through (8). For mixtures containing HFC-125 or HFC-152a, high refrigerant concentration data was fitted to equations (9) through (11).

Multivariate correlation coefficients, $\sigma$, have been calculated to measure the fit of the regression equation to the data. The coefficients are derived from the following expression:

$$
\sigma=\frac{\sqrt{\Sigma\left(y_{t}-y_{a v}\right)^{2}-\Sigma\left(y_{i}-y_{c}\right)^{2}}}{\Sigma\left(y_{t}-y_{a v}\right)^{2}}
$$

where

$$
\begin{array}{ll}
\mathrm{y}_{\mathrm{i}} & =\text { experimental data point } \\
\mathrm{y}_{\mathrm{c}} & =\text { calculated data point } \\
\mathrm{y}_{\mathrm{av}} & =\text { average of experimental data points }
\end{array}
$$




\section{Equations for Low Refrigerant Concentrations}

Dynamic viscosity $(\mu)$ is represented by a modified Walther equation:

$$
\begin{aligned}
\log \{\log (\mu+0.7)\}= & \left\{a_{1}+a_{2} \log (\mathrm{T})+\mathrm{a}_{3} \log ^{2}(\mathrm{~T})\right\} \\
& +\omega\left\{\mathrm{a}_{4}+\mathrm{a}_{5} \log (\mathrm{T})+\mathrm{a}_{6} \log ^{2}(\mathrm{~T})\right\} \\
& +\omega^{2}\left\{\mathrm{a}_{7}+\mathrm{a}_{8} \log (\mathrm{T})+\mathrm{a}_{9} \log ^{2}(\mathrm{~T})\right\}
\end{aligned}
$$

Vapor pressure $(\mathrm{P})$ is represented by:

(2)

$$
\begin{aligned}
& \left\{a_{1}+a_{2} T+a_{3} T^{2}\right\} \\
& +\omega\left\{a_{4}+a_{5} T+a_{6} T^{2}\right\} \\
& +\omega^{2}\left\{a_{7}+a_{8} T+a_{9} T^{2}\right\}
\end{aligned}
$$

Density ( $p$ ) is represented by:

$$
\begin{aligned}
\rho \quad=\quad & \left\{a_{1}+a_{2} T+a_{3} T^{2}\right\} \\
& +\omega\left\{a_{4}+a_{5} T+a_{6} T^{2}\right\} \\
& +\omega^{2}\left\{a_{7}+a_{8} T+a_{9} T^{2}\right\}
\end{aligned}
$$

Kinematic viscosity $(v)$ is represented by:

$$
\begin{aligned}
\log \{\log (v+0.7)\}= & \left\{a_{1}+a_{2} \log (T)+a_{3} \log ^{2}(T)\right\} \\
& +\omega\left\{a_{4}+a_{5} \log (T)+a_{6} \log ^{2}(T)\right\} \\
& +\omega^{2}\left\{a_{7}+a_{8} \log (T)+a_{9} \log ^{2}(T)\right\}
\end{aligned}
$$

where:

$\begin{array}{ll}\mu & \text { dynamic (absolute) viscosity, centipoise } \\ \mathrm{P} & \text { pressure, kilopascals } \\ \rho & \text { density, gram/cubic centimeter } \\ \mathrm{V} & \text { kinematic viscosity, centistoke } \\ \mathrm{T} & \text { temperature, Kelvin } \\ \omega & \text { mass fraction refrigerant } \\ \mathrm{a}_{1} \ldots \mathrm{a}_{9} & \text { constants }\end{array}$


Equations for High Concentration Refrigerants (other than HFC-125 and HFC-152a)

Dynamic viscosity $(\mu)$ is represented by a modified Walther equation:

$$
\begin{aligned}
\log (\mu)= & \left\{a_{1}+a_{2} / T+a_{3} / T^{2}\right\} \\
& +\omega\left\{a_{4}+a_{5} / T+a_{6} / T^{2}\right\} \\
& +\omega^{2}\left\{a_{7}+a_{8} / T+a_{9} / T^{2}\right\}
\end{aligned}
$$

Vapor pressure $(\mathrm{P})$ is represented by:

(6) $\quad \log (\mathrm{P})=\left\{\mathrm{a}_{1}+\mathrm{a}_{2} / \mathrm{T}+\mathrm{a}_{3} / \mathrm{T}^{2}\right\}$

$$
\begin{aligned}
& +\omega\left\{\mathrm{a}_{4}+\mathrm{a}_{5} / \mathrm{T}+\mathrm{a}_{6} / \mathrm{T}^{2}\right\} \\
& +\omega^{2}\left\{\mathrm{a}_{7}+\mathrm{a}_{8} / \mathrm{T}+\mathrm{a}_{9} / \mathrm{T}^{2}\right\}
\end{aligned}
$$

Density ( $\rho)$ is represented by:

$$
\begin{aligned}
=\quad & \left\{a_{1}+a_{2} T_{r}+a_{3} T_{r}^{2}\right\} \\
& +\omega\left\{a_{4}+a_{5} T_{r}+a_{6} T_{r}^{2}\right\} \\
& +\omega^{2}\left\{a_{7}+a_{8} T_{r}+a_{9} T_{r}^{2}\right\}
\end{aligned}
$$

Kinematic viscosity $(v)$ is represented by:

$$
\begin{aligned}
\log (v)=\quad & \left\{a_{1}+a_{2} / T+a_{3} / T^{2}\right\} \\
& +\omega\left\{a_{4}+a_{5} / T+a_{6} / T^{2}\right\} \\
& +\omega^{2}\left\{a_{7}+a_{8} / T+a_{9} / T^{2}\right\}
\end{aligned}
$$

where:

$\begin{array}{ll}\mu & \text { dynamic (absolute) viscosity, centipoise } \\ \mathrm{P} & \text { pressure, kilopascals } \\ \rho & \text { density, gram/cubic centimeter } \\ \nu & \text { kinematic viscosity, centistoke } \\ \mathrm{T} & \text { temperature, Kelvin } \\ \mathrm{T}_{\mathrm{c}} & \text { critical temperature, Kelvin } \\ \mathrm{T}_{\mathrm{r}} & 1-\mathrm{T} / \mathrm{T}_{\mathrm{c}} \\ \omega & \text { mass fraction refrigerant } \\ \log & \text { logarithm to the base } 10 \\ \mathrm{a}_{1} \ldots \mathrm{a}_{9} & \text { constants }\end{array}$


Kinematic viscosity $(v)$ is represented by:

(9)

$$
\begin{array}{lll}
\log \left(v_{100}\right) & =\mathrm{a}_{1}+\mathrm{a}_{2} / \mathrm{T}+\mathrm{a}_{3} / \mathrm{T}^{2} \\
\log \left(v_{90}\right) & =\mathrm{a}_{4}+\mathrm{a}_{5} / \mathrm{T}+\mathrm{a}_{6} / \mathrm{T}^{2} \\
\log \left(v_{80}\right) & =\ldots \mathrm{a}_{7}+\mathrm{a}_{8} / \mathrm{T}+\mathrm{a}_{9} / \mathrm{T}^{2}
\end{array}
$$

Vapor pressure $(\mathrm{P})$ is given by:

(10) $\begin{array}{lll}\log \left(\mathrm{P}_{100}\right) & = & \mathrm{a}_{1}+\mathrm{a}_{2} / \mathrm{T}+\mathrm{a}_{3} / \mathrm{T}^{2} \\ \log \left(\mathrm{P}_{90}\right) & = & \mathrm{a}_{4}+\mathrm{a}_{5} / \mathrm{T}+\mathrm{a}_{6} / \mathrm{T}^{2} \\ \log \left(\mathrm{P}_{80}\right) & = & \mathrm{a}_{7}+\mathrm{a}_{8} / \mathrm{T}+\mathrm{a}_{9} / \mathrm{T}^{2}\end{array}$.

Density $(\rho)$ is given by:

$$
\begin{array}{lll}
\rho_{100} & = & a_{1}+a_{2} T=a_{3} T^{2} \\
\rho_{90} & = & a_{4}+a_{5} T=a_{6} T^{2} \\
\rho_{80} & = & a_{7}+a_{8} T=a_{9} T^{2}
\end{array}
$$

where:

$\begin{array}{ll}v & \text { kinematic viscosity, centistokes } \\ \mathrm{P} & \text { kinematic viscosity, centistokes } \\ \rho & \text { density, gram/cubic centimeter } \\ \log & \text { logarithm to the base } 10 \\ \mathrm{~T} & \text { temperature, Kelvin } \\ \mathrm{a}_{1} \ldots \mathrm{a}_{9} & \text { constants }\end{array}$

the subscripts 100,90 , and 80 refer to the mass fraction refrigerant -

Mr. Henderson's report contains tables with viscosity, solubility and density parameters density charts and Daniel charts for each of the refrigerant lubricant mixtures measured. Tables 24-1 and 24-2 and Figures 24-1 through 24-2 are samples of the summaries for HFC-134a and ISO $68 \mathrm{cSt}$ pentaerythritol ester mixed-acid mixtures. 
Table 24-1. Viscosity, Solubility and Density Parameters HFC-134a/ISO 68 Pentaerythritol Ester Mixed-Acid. (Low Refrigerant Concentrations)

\begin{tabular}{|c|c|c|c|c|}
\hline Coefficient & $\begin{array}{c}\text { Dynamic } \\
\text { Viscosity } \\
\text { (eq. 1) }\end{array}$ & $\begin{array}{c}\text { Vapor } \\
\text { Pressure } \\
\text { (eq. 2) }\end{array}$ & $\begin{array}{c}\text { Density } \\
\text { (eq. 3) }\end{array}$ & $\begin{array}{c}\text { Kinematic } \\
\text { Viscosity } \\
\text { (eq. 4) }\end{array}$ \\
\hline $\mathrm{a}_{1}$ & $1.05204 \mathrm{E}+1$ & $1.16900 \mathrm{E}+3$ & 1.20668 & $1.02380 \mathrm{E}+1$ \\
\hline $\mathrm{a}_{2}$ & -4.11222 & -7.39656 & $-9.16226 \mathrm{E}-4$ & -3.99658 \\
\hline $\mathrm{a}_{3}$ & 0 & $1.16084 \mathrm{E}-2$ & $3.28702 \mathrm{E}-7$ & 0 \\
\hline $\mathrm{a}_{4}$ & $-1.17928 \mathrm{E}+1$ & $-5.87454 \mathrm{E}+3$ & $3.67221 \mathrm{E}-1$ & $-1.20459 \mathrm{E}+1$ \\
\hline $\mathrm{a}_{5}$ & 4.18034 & $-5.09869 \mathrm{E}+1$ & $4.48469 \mathrm{E}-5$ & 4.27634 \\
\hline $\mathrm{a}_{6}$ & 0 & $2.65209 \mathrm{E}-1$ & $-1.13568 \mathrm{E}-6$ & 0 \\
\hline$a_{7}$ & $2.55320 \mathrm{E}+1$ & $1.79697 \mathrm{E}+5$ & $8.22484 \mathrm{E}-1$ & $2.57746 \mathrm{E}+1$ \\
\hline$a_{8}$ & -9.93423 & $-1.02803 \mathrm{E}+3$ & $-4.69511 \mathrm{E}-3$ & $-1.00588 \mathrm{E}+1$ \\
\hline$a_{9}$ & 0 & 1.39473 & $5.95292 \mathrm{E}-6$ & 0 \\
\hline$\sigma$ & 0.9993 & 0.9998 & 0.9999 & 0.9993 \\
\hline
\end{tabular}

Figure 24-1. Density of HFC-134a/ISO 68 Pentaerythritol Ester Mixed-Acid. (Low Refrigerant Concentrations)

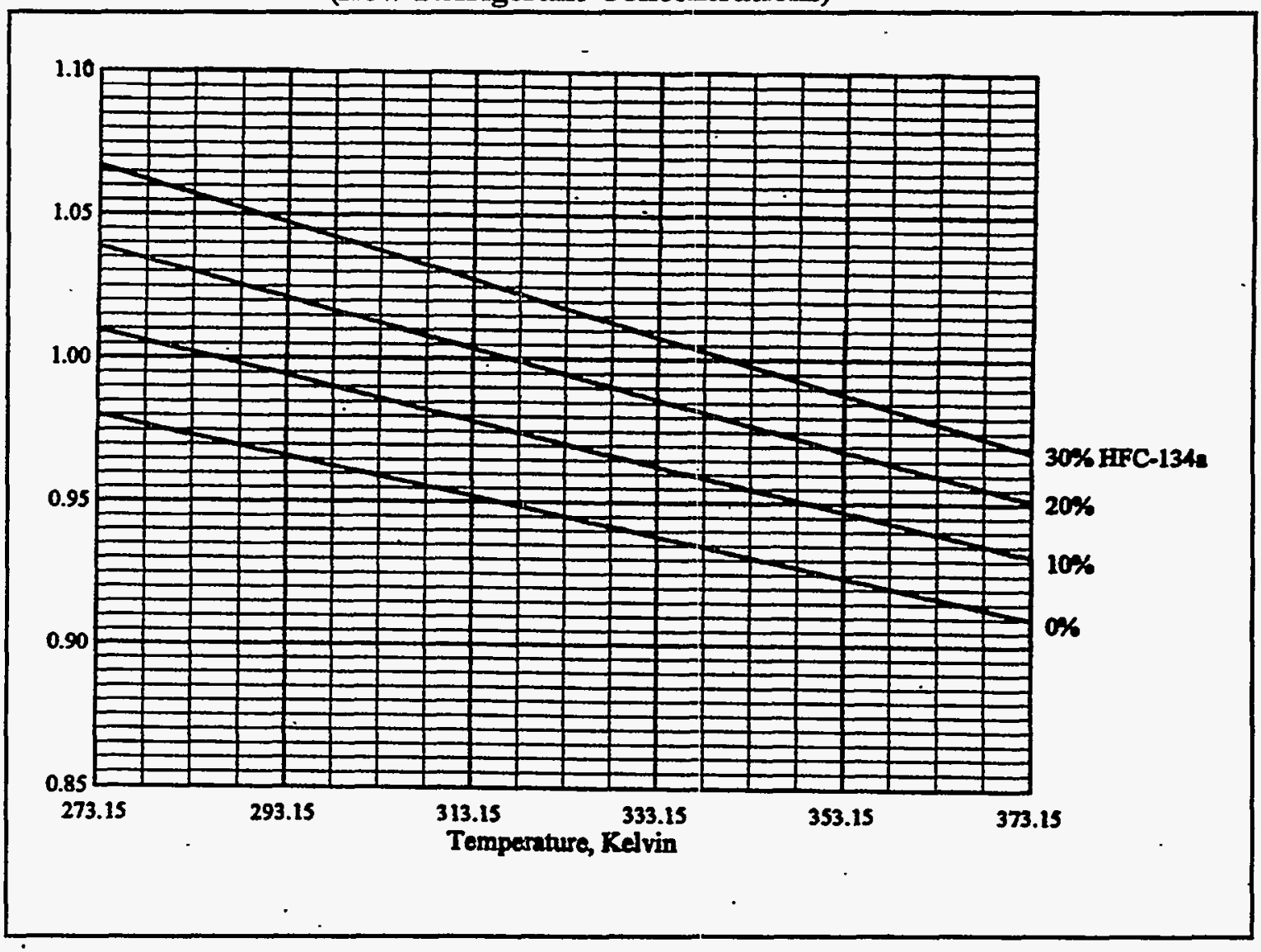


Figure 24-2. Viscosity and Solubility of

HFC-134a/ISO 68 Pentaerythritol Ester Mixed-Acid.

(Low Refrigerant Concentrations)

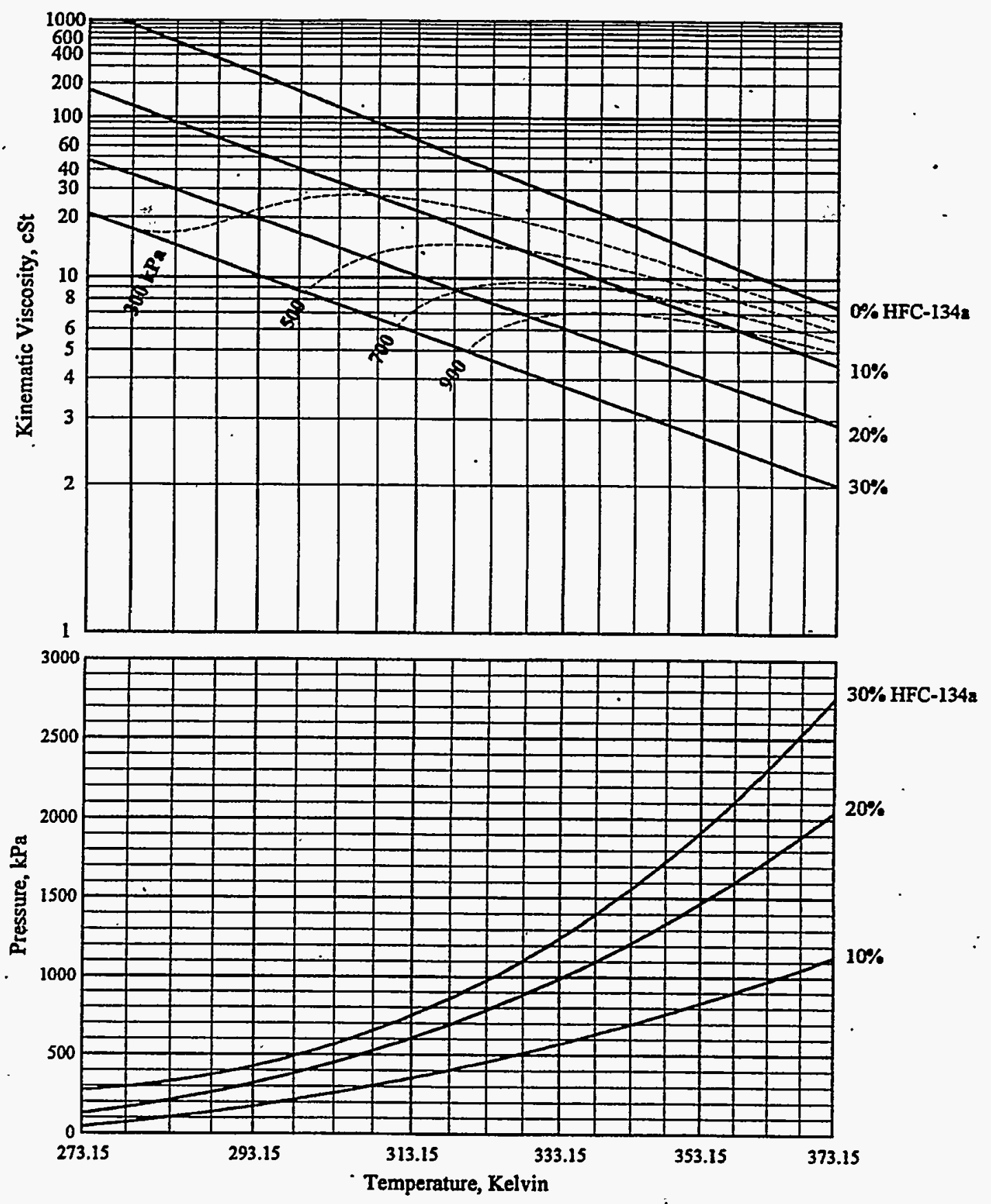


Table 24-2. Viscosity, Solubility and Density Parameters HFC-134a/ISO 68 Pentaerythritol Ester Mixed-Acid. (High Refrigerant Concentrations)

\begin{tabular}{|c|c|c|c|c|}
\hline Coefficient & $\begin{array}{c}\text { Dynamic } \\
\text { Viscosity } \\
\text { (eq. 1) }\end{array}$ & $\begin{array}{c}\text { Vapor } \\
\text { Pressure } \\
\text { (eq. 2) }\end{array}$ & $\begin{array}{c}\text { Density } \\
\text { (eq. 3) }\end{array}$ & $\begin{array}{c}\text { Kinematic } \\
\text { Viscosity } \\
\text { (eq. 4) }\end{array}$ \\
\hline$a_{1}$ & $-154267 \mathrm{E}-1$ & 4.93501 & $-6.03916 \mathrm{E}-2$ & $6.70804 \mathrm{E}-1$ \\
\hline$a_{2}$ & $-1.30839 \mathrm{E}+2$ & $-3.90373 \mathrm{E}+2$ & -2.50554 & $-1.13257 \mathrm{E}+2$ \\
\hline$a_{3}$ & $1.89773 \mathrm{E}+5$ & $-9.75213 \mathrm{E}+4$ & 1.66826 & $1.90778 \mathrm{E}+5$ \\
\hline$a_{4}$ & -1.35162 & 1.98743 & 1.30515 & -2.54713 \\
\hline$a_{5}$ & $-1.84121 \mathrm{E}+2$ & $-6.29632 \mathrm{E}+2$ & 5.39346 & $-2.84865 \mathrm{E}+2$ \\
\hline$a_{6}$ & $-9.66564 \mathrm{E}+4$ & $5.83317 \mathrm{E}+4$ & -4.46156 & $-9.66465 \mathrm{E}+4$ \\
\hline$a_{7}$ & $-7.89904 \mathrm{E}-1$ & $9.65170 \mathrm{E}-1$ & $-3.42380 \mathrm{E}-1$ & $3.26527 \mathrm{E}-1$ \\
\hline$a_{8}$ & $8.13268 \mathrm{E}+2$ & $-7.75785 \mathrm{E}+2$ & -1.35909 & $5.19287 \mathrm{E}+2$ \\
\hline$a_{9}$ & $-1.0393 \mathrm{E}+5$ & $1.2343 \mathrm{E}+5$ & 2.26507 & $-6.55262 \mathrm{E}+4$ \\
\hline$\sigma$ & 0.9999 & 0.9999 & 0.9999 & 0.9999 \\
\hline
\end{tabular}

Figure 24-3. Density of HFC-134a/ISO 68 Pentaerythritol Ester Mixed-Acid. (High Refrigerant Concentrations)

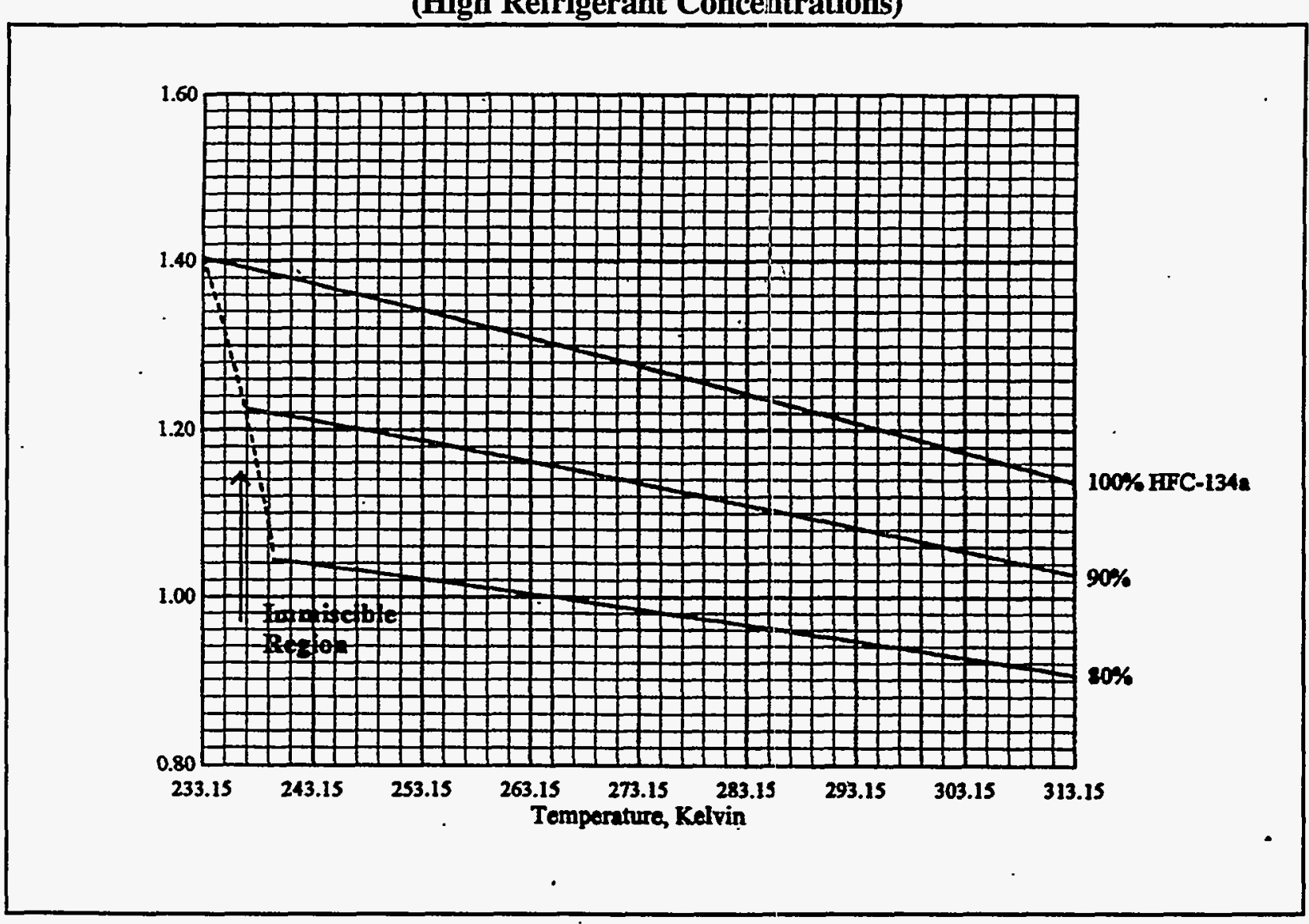


Figure 24-4. Viscosity and Solubility of

HFC-134a/ISO 68 Pentaerythritol Ester Mixed-Acid. (High Refrigerant Concentrations)

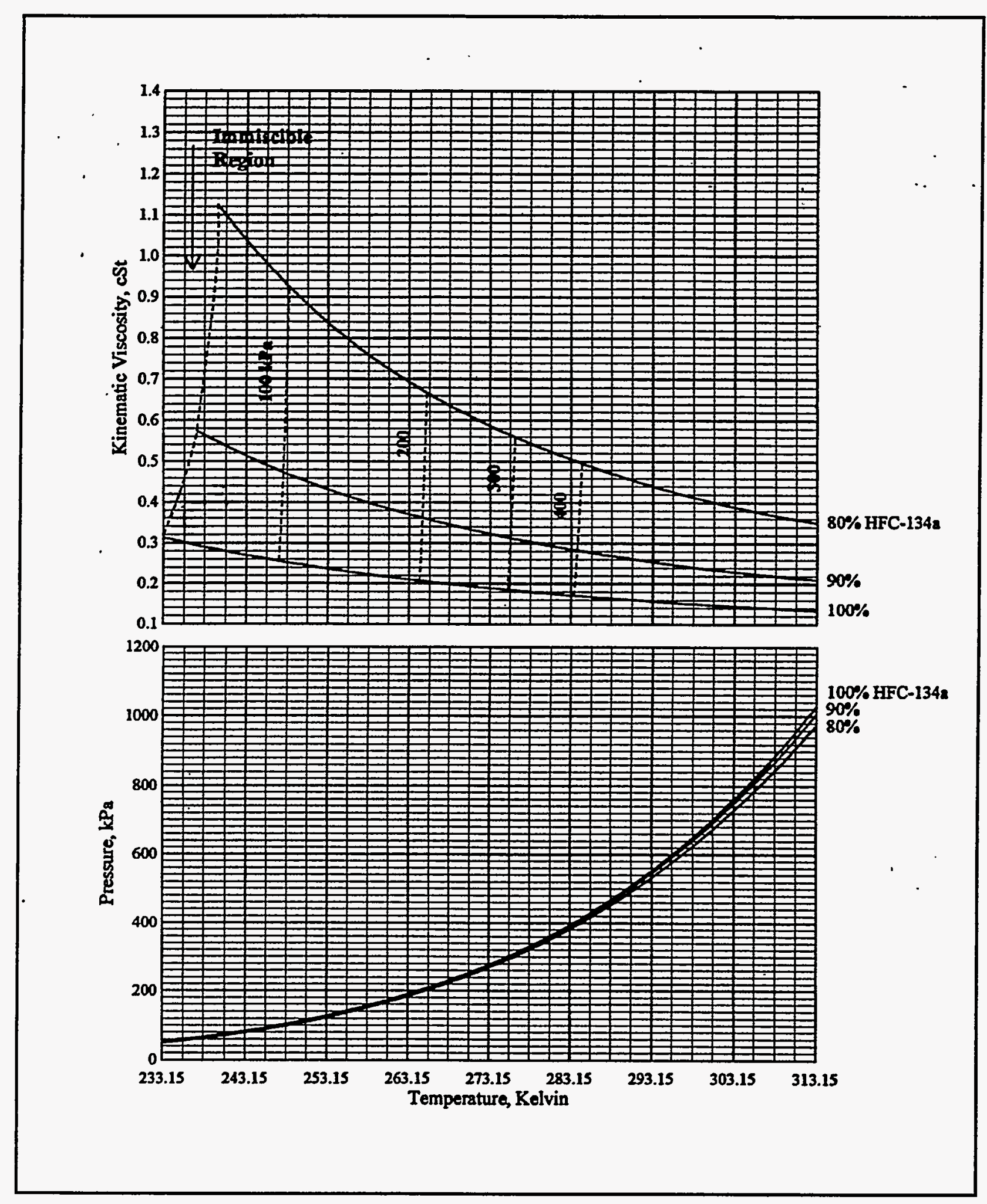




\section{MEASUREMENT OF VISCOSITY, DENSITY, AND GAS SOLUBIILITY \\ OF REFRIGERANT AZEOTROPES AND BLENDS}

\section{Objective:}

To measure the viscosity, density, and solubility of three refrigerant blends that may potentially replace HCFC-22 or R-502.

\section{Results:}

Imagination Resources, Inc. completed this research under contract with ARTI. Detailed results of the study are presented in the final report, DOE/CE/23810-46, Measurement of Viscosity, Density, and Gas Solubility of Refrigerant Blends, by Richard C. Cavestri, $\mathrm{PhD}$, 15 March 1995.

Viscosity, solubility, and density data are reported for the following refrigerant-lubricant mixtures:

Baseline refrigerant-lubricant mixtures:

- HCFC-22 and Suniso ${ }^{\otimes}$ 3GS mineral oil

- R-502 and Suniso ${ }^{\circledR} 3$ GS mineral oil

Single-component refrigerant mixtures:

- HFC-32 and 32 ISO mixed-acid polyolester

- HFC-32 and 32 ISO branched-acid polyolester

- HFC-125 and 32 ISO mixed-acid polyolester

- HFC-125 and 32 ISO branched-acid polyolester

- HFC-134a and 32 ISO mixed-acid polyolester.

- HFC-134a and 32 ISO branched-acid polyolester

- HFC-143a and 32 ISO mixed-acid polyolester

- HFC-143a and 32 ISO branched-acid polyolester

Blend refrigerant-lubricant mixtures:

- R-404A (HFC-125/HFC-143a/HFC-134a; 44/52/4\%) and 32 ISO mixed-acid polyolester

- R-404A and 32 ISO branched-acid polyolester

- R-407C (HFC-32/HFC-125/HFC-134a; 23/25/52\%) and 32 ISO mixed-acid polyolester

- R-407C and 32 ISO branched-acid polyolester

- R-410A (HFC-125/HFC-143a; 50/50\%) and 32 ISO mixed-acid polyolester

- R-410A and 32 ISO branched-acid polyolester 
For each refrigerant-lubricant pair, the report graphically presents data from -20 or $0^{\circ} \mathrm{C}$ to $125^{\circ} \mathrm{C}\left(-4\right.$ or $32^{\circ} \mathrm{F}$ to $\left.257^{\circ} \mathrm{F}\right)$ for a pressure range of 69 to $1,724 \mathrm{kPa}$ (10 to $\left.250 \mathrm{psia}\right)$ :

- viscosity and pressure vs. temperature at constant refrigerant concentrations $(0,10$, $20,30,40,50$, and $60 \%$ );

- density vs. temperature;

- viscosity vs. temperature; and

- viscosity and gas solubility ys. pressure (at temperature intervals of $0,20,40,60$, 80,100 , and $125^{\circ} \mathrm{C}$ ). Refrigerant blends include gas fractionation information.

Figure 25-1 is an example of the presentation plot for viscosity vs. gas solubility of R404A (HFC-125/HFC-143a/HFC-134a; 44/52/4\%) at $125^{\circ} \mathrm{C}\left(257^{\circ} \mathrm{F}\right)$. It depicts the relationship between reduction in refrigerant-lubricant viscosity with increasing concentration of refrigerant. Gas fractionation of the individual constituents within the refrigerant blend are also indicated.

Figure 25-1. Viscosity, Solubility and Gas Fractionation 32 ISO VG Mixed Acid Polyolester with $R-404 A$ at $125^{\circ} \mathrm{C}$

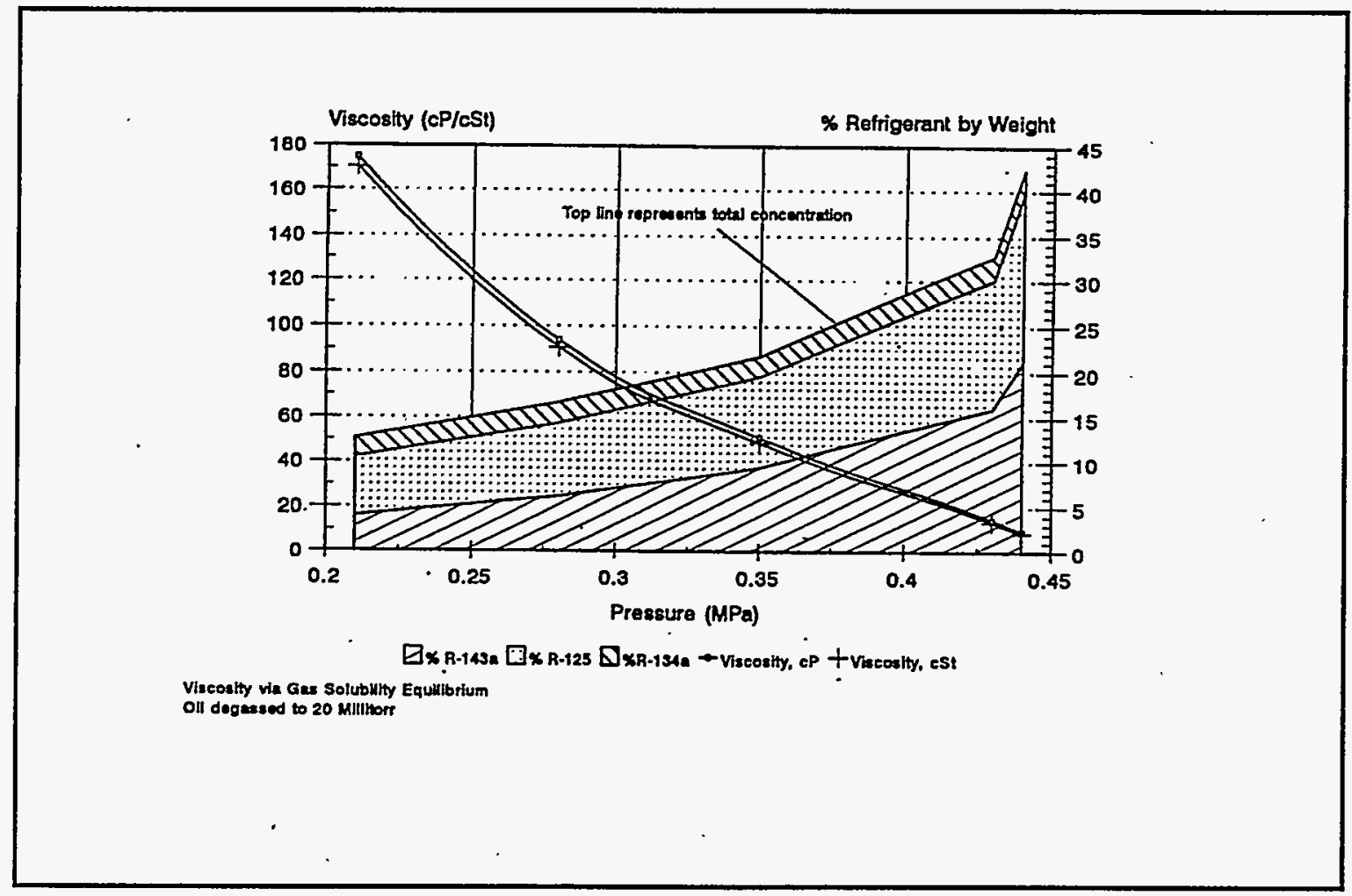




\section{COMPATIBILITY OF REFRIGERANTS AND LUBRICANTS}

WITH MOTOR MATERIALS

\section{Objective:}

To provide information on the compatibility of motor materials with potential substitutes for $\mathrm{CFC}$ refrigerants and with suitable lubricants.

\section{Results:}

The Trane Company has completed this research under contract with ARTI. Detailed results are presented in the final report, DOE/CE/23810-13, Compatibility of Refrigerants and Lubricants with Motor Materials, May 1993, by Robert Doerr, PhD, Stephen Kujak and Todd Waite (Vol I - RDB \#3857, 166 pages; Vol II - RDB \#3858, 270 pages; Vol III - RDB \#3859, 370 pages).

Results from the project indicate that most materials used in current hermetic motors are compatible with the test refrigerant-lubricant combinations.

The project examined the compatibility of twenty-four hermetic motor materials with eleven pure refrigerants and seventeen refrigerant-lubricant combinations. Motor materials tested included three types of magnet wires, six wire varnishes, six sheet insulations, three sleeving insulations, three tie tapes, two lead wire insulations and one tie cord. A number physical property measurements were performed on samples of each test material before and after its exposure to the refrigerants and refrigerant-lubricant mixtures.

\section{$\underline{\text { Refrigerants }}$}

HCFC-22@ $90^{\circ} \mathrm{C}\left(194^{\circ} \mathrm{F}\right)$

HCFC-123 @ 90 $90^{\circ} \mathrm{C}\left(194^{\circ} \mathrm{F}\right)$

HCFC-124@ $90^{\circ} \mathrm{C}\left(194^{\circ} \mathrm{F}\right)$

HCFC-142b @ $90^{\circ} \mathrm{C}\left(194^{\circ} \mathrm{F}\right)$

HFC-152a @ 90 $90^{\circ} \mathrm{C}\left(194^{\circ} \mathrm{F}\right)$

HFC-134a @ 90 $\mathrm{C}\left(194^{\circ} \mathrm{F}\right)$
$\mathrm{HFC}-134 @ 90^{\circ} \mathrm{C}\left(194^{\circ} \mathrm{F}\right)$

HFC-32@ $60^{\circ} \mathrm{C}\left(140^{\circ} \mathrm{F}\right)$

HFC-125@60 $\mathrm{C}\left(140^{\circ} \mathrm{F}\right)$

HFC-143a @ 60 $0^{\circ} \mathrm{C}\left(140^{\circ} \mathrm{F}\right)$

HFC-245ca @ $121^{\circ} \mathrm{C}\left(250^{\circ} \mathrm{F}\right)$ 


\section{Refrigerant-Lubricant Combinations at $127^{\circ} \mathrm{C}\left(260^{\circ} \mathrm{F}\right)$}

HCFC-22/mineral oil (ISO 32 )

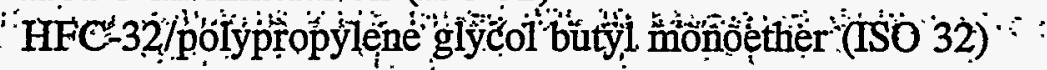

HFC-32/pentaerythritol ester branched-acid (ISO 32)

HCFC-124/alkylbenzene (ISO 32)

HFC-125/polypropylene glycol butyl monoether (ISO 32)

HFC-125/modified polyalkylene glycol (ISO 32)

HFC-125/pentaerythritol ester branched-acid (ISO 32)

HFC-134/pentaerythritol ester branched-acid (ISO 32)

HFC-134a/polypropylene glycol butyl monoether (ISO 32)

HFC-134a/polypropylene glycol diol (ISO 32)

HFC-134a/modified polyalkylene glycol (ISO 32)

HFC-134a/pentaerythritol ester mixed-acid (ISO 22)

HFC-134a/pentaerythritol ester branched-acid (ISO 32)

HCFC-142b/alkylbenzene (ISO 32)

HFC-143a/pentaerythritol ester branched-acid (ISO 32)

HFC-245ea/pentaerythritol ester branched-acid (ISO 32)

HFC-152a/alkylbenzene

\section{Motor Materials Evaluations}

$\begin{array}{ll}\begin{array}{l}\text { Varnish } \\ \text { weight change }\end{array} & \begin{array}{l}\text { Spiral Wrapped } \\ \text { weight change } \\ \text { break loan stren }\end{array} \\ \begin{array}{l}\text { Lead Wire } \\ \text { weight change } \\ \text { dielectric strength }\end{array} & \begin{array}{l}\text { Sheet Insulation } \\ \text { weight change } \\ \text { tensile strength } \\ \text { elongation } \\ \text { dielectric streng }\end{array} \\ \begin{array}{l}\text { Tie Cord } \\ \text { weight change } \\ \text { break load strength }\end{array} \\ \begin{array}{l}\text { Magnet Wire/V.arnish } \\ \text { bond strength } \\ \text { burnout resistance } \\ \text { dielectric strength }\end{array} & \begin{array}{l}\text { Tapes } \\ \text { weight change }\end{array} \\ & \end{array}$

There were no compatibility concerns with any of the three magnet wires tested. Most of the test varnishes were compatible with the refrigerant-lubricant mixtures. One of the six tested varnishes, the Sterling Y-833 varnish (100\% solids VPI epoxy), raised compatibility concerns. It was considered incompatible with HCFC-123 and exhibited physical changes 
when tested with HCFC-22. The varnish became soft, limp and crazed after the 500-hour exposure to HCFC-123. The varnish also became severely crazed and limp after exposure to HCFC-22. Varnish is used in hermetic motors to bind motor wire windings and to prevent wire-to-wire rubbing from stripping away the insulating coat and electrically shorting the motor.

Only one of the three tapes tested displayed any compatibility problems. The glass/acrylic tape was considered incompatible with HCFC-123. After exposure, it exhibited a large weight loss, turned green in color, rolled up and separated from its backing. Compatibility concerns also arose in tests with nine of the seventeen refrigerant-lubricant mixtures. After exposure, the tape curled up and its backing easily rubbed off. However, when the tape was heated for an addition 24 hours at $150^{\circ} \mathrm{C}\left(302^{\circ} \mathrm{F}\right)$ it regained its original unexposed form.

Three of the six sleeving materials tested had compatibility concerns. The laminating adhesive in the Nomex, Mylar, and Nomex/Mylar sleeving insulations weakened after exposure to HCFC-22/mineral oil and/or HCFC-124/alkylbenzene mixtures. However, it was noted that these materials have been used in HCFC-22/mineral oil applications for 20 to 30 years without equipment reliability problems.

Sheet insulation materials raised more compatibility concerns than any of the other materials tested. The Nomex/Mylar/Nomex was considered incompatible with the HFC134a/polypropylene glycol diol (PAG-diol) mixture. The adhesive which bonds the layers together dissolved. Pockets of delamination also resulted after the material was exposed to five of the pure refrigerants and eleven of the refrigerant-lubricant mixtures. The material also lost flexibility or became brittle after exposure to four other refrigerantlubricant mixtures.

Dacron/Mylar/Dacron sheet insulation was also considered incompatible with the HFC134a/PAG-diol mixture because of dissolution of the laminating adhesive. Additional compatibility concerns were raised due to excessive weight loss after exposure of the material to HCFC-22, HFC-245ca, HFC-134a/polypropylene glycol (PAG-butyl monoether) and HFC-134a/modified PAG mixtures. The material also experienced embrittlement and/or lost flexibility after exposure to four other refrigerant-lubricant mixtures.

Likewise, Melinex 228 and Mylar MO raised compatibility concerns due to embrittlement or loss of flexibility after exposure to four refrigerant-lubricant mixtures which contained mineral oil or alkylbenzene. Nomex 410 and Nomex 418 raised compatibility concerns because of excessive weight loss after exposure to HFC-125. 


\section{COMPATIBILITY OF REFRIGERANTS AND LUBRICANTS . WITH ELASTOMERS}

\section{Objectives:}

- To provide compatibility information for elastomers with potential substitutes for CFC refrigerants and with suitable lubricants.

- To obtain data on changes in the physical and mechanical properties of selected elastomers after thermal aging in refrigerant-lubricant mixtures.

Results:

The University of Akron has completed this research under contract with ARTI. Detailed results are presented in the final report, DOE/CE/23810-14, Compatibility of Refrigerants and Lubricants with Elastomers, January 1994, Gary R. Hamed, PhD, Robert H. Seiple, and Orawan Taikum (RDB \# 4501, 538 pages).

This research project examined the compatibility of ten refrigerant and seven lubricants with ninety-five elastomeric materials:

$\begin{array}{ll}\text { Refrigerants } & \underline{\text { Lubricants }} \\ \text { HCFC-22 } & \begin{array}{l}\text { naphthenic mineral oil (ISO 32) } \\ \text { alkylbenzene (ISO 32) } \\ \text { HCFC-123 } \\ \text { HCFC-124 }\end{array} \\ \text { polypropylene glycol butyl monoether (ISO 32) } \\ \text { HFC-32 } & \begin{array}{l}\text { polypropylene glycol diol (ISO 32) } \\ \text { modified polyglycol (ISO 32) }\end{array} \\ \text { HFC-125 } & \text { pentaerythritol ester, mixed-acid (ISO 22) } \\ \text { HFC-134 } & \text { pentaerythritol ester, branched-acid (ISO 32) } \\ \text { HFC-134a } & \\ \text { HFC-143a } & \\ \text { HFC-152a } & \end{array}$




\section{Elastomer Families}

butyl polypropylene TPE (1 type)

butyl rubbers (7 types)

chlorinated polyethylenes ( 3 types)

chlorosulfonated polyethylenes (5 types)

epichlorohydrin based rubbers (6 types)

ethylene acrylic elastomers ( 2 types)

ethylene propylene rubbers (3 types)

ethylene propylene diene rubbers (5 types)

fluorinated rubbers (7 types) nitrile rubbers (10 types)

polychloroprenes ( 2 types)

polyisoprenes (3 types)

polysulfide rubbers (4 types)

polyurethanes ( 7 types)

silicones (5 types)

styrene butadiene rubbers (4 types).

thermoplastic elastomers (11 types)

$$
\text { plus, ten industry-supplied gaskets of various compositions. }
$$

Swell behavior of elastomer samples were determined by comparing pre-exposure sample measurements for weight, thickness and diameter with their measurements after exposure. As indicated above, these elastomeric formulations included general purpose and specialty thermoset and thermoplastic elastomers.

Refrigerant Immersion Studies: Elastomer samples were completely immersed in the test refrigerant, sealed in a pressure vessel and maintained at room temperature (ambient) for 14 days. In situ diameter changes were determined using a traveling microscope after 24hour, 72-hour and 14-day exposures. Following the 14 day exposures, the samples were remeasured 2 hours and 24 hours after they were removed from the pressure vessels.

In reviewing the results, the following general statements can be made concerning in situ swelling measurements after the 14 day exposures:

- samples exposed to HCFC-123 had the largest swell,

- samples exposed to HCFC-22, HCFC-124, HCFC-142b had moderate swell,

- samples exposed to HFC-32, HFC-125, HFC-134, HFC-134a, HFC-143a, and HFC-152a had the least swell.

Refer to Table 27-1 for a relative comparison of in situ swelling results. 
Lubricant Immersion Studies: Elastomer samples were completely immersed in the test lubricant, sealed in a glass vessel and then heated at $60^{\circ} \mathrm{C}\left(140^{\circ} \mathrm{F}\right)$ for 14 days. Sample diameters were measured in situ after 24 hours of exposure. The elastomer samples were also measured for weight, thickness and diameter immediately after the 14-day exposure and then again 24 hours after removal.

Several of the elastomeric compositions, including some of the industry-supplied gaskets, were. resistant to swelling in all of the lubricants. These included rubbers from the epichlorohydrin, nitrile, polysulfide rubber, and thermoplastic elastomer families. Refer to Table 27-2 for a relative comparison of the in situ swelling results.

Refrigerant-Lubricant Thermal Aging Tests: Based on the results of the separate lubricant and refrigerant studies, twenty-five elastomeric samples were selected for inclusion in refrigerant-lubricant thermal aging tests. These elastomers were individually immersed in seventeen separate refrigerant-lubricant mixtures for 14 days at $100^{\circ} \mathrm{C}\left(212{ }^{\circ} \mathrm{F}\right)$. Depending on the refrigerant-lubricant combination, the refrigerant weight percent varied from $20 \%$ to $50 \%$ concentration to maintain a vapor pressure of $275-300$ psia. After the 14-day exposures, dimensional, hardness, and tensile values of the exposed elastomers were obtained and compared to those of non-aged specimens.

As a general trend, it was found that the tensile strengths of the aged elastomers were inversely related to the amount of swelling they exhibited after aging in the refrigerantlubricant mixtures. When swelling was large, elastomer tensile strength decreased dramatically. However, in some cases, when swelling was slight or negative (i.e., shrinkage from material extraction) tensile strength increased after aging. In all cases, filled rubbers showed less change of tensile strength after aging compared to unfilled counterparts. 
Table 27-1. Relative in situ Elastomer Swelling in Refrigerants

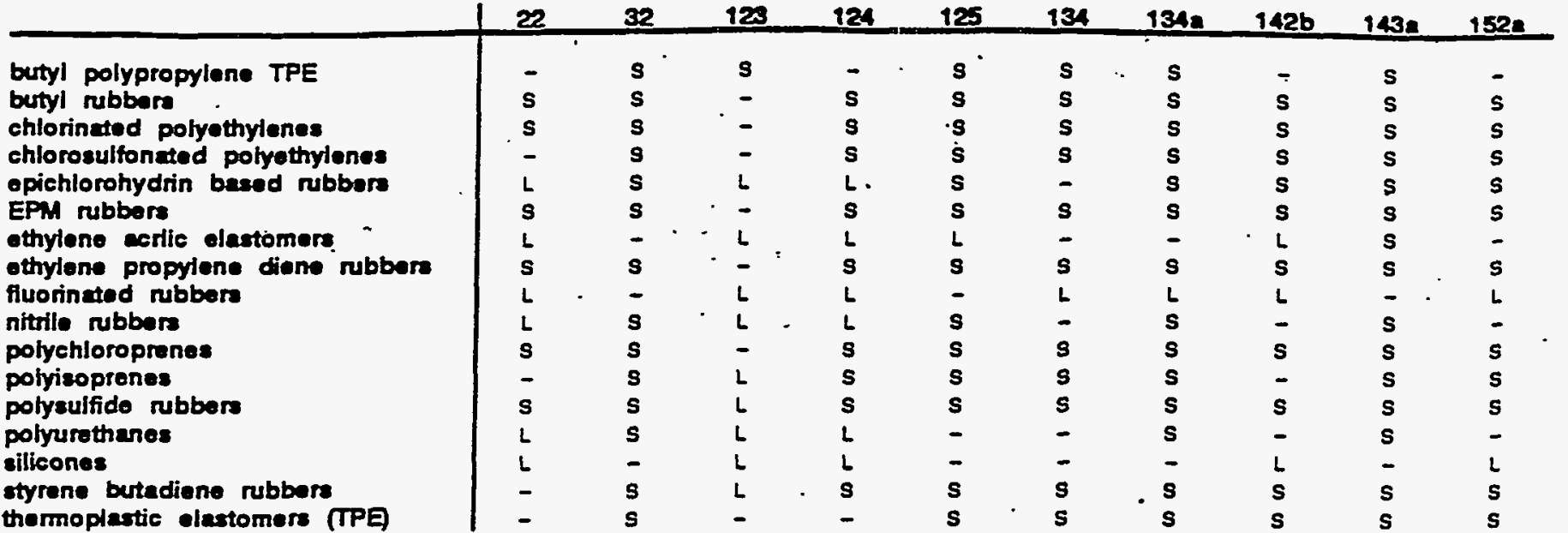

Table 27-2. Relative in site Elastomer Swelling in Lubricants.

\begin{tabular}{|c|c|c|c|c|c|c|c|}
\hline & $\mathbf{A B}$ & MO & FEaA & PENA & Pregen & PPCD & NPS \\
\hline 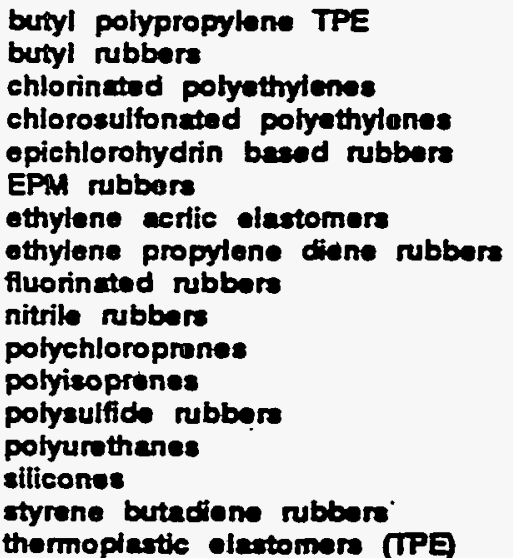 & $\begin{array}{l}- \\
L \\
S \\
- \\
S \\
L \\
- \\
L \\
S \\
S \\
- \\
L \\
S \\
- \\
- \\
L \\
\end{array}$ & $\begin{array}{l}- \\
L \\
- \\
-E \\
S \\
L \\
- \\
L \\
S \\
S \\
- \\
L \\
S \\
S \\
- \\
L \\
-\end{array}$ & $\begin{array}{l}\mathbf{s} \\
\mathbf{s} \\
- \\
- \\
- \\
\mathbf{s} \\
\mathbf{L} \\
\mathbf{S} \\
\mathbf{L} \\
- \\
- \\
- \\
\mathbf{S} \\
\mathbf{S} \\
- \\
- \\
\mathbf{S}\end{array}$ & $\begin{array}{l}\mathbf{s} \\
\mathbf{s} \\
- \\
- \\
- \\
\mathbf{S} \\
\mathbf{L} \\
\mathbf{S} \\
- \\
- \\
\mathbf{L} \\
\mathbf{S} \\
\mathbf{S} \\
- \\
\mathbf{S}\end{array}$ & $\begin{array}{l}s \\
s \\
s \\
s \\
S \\
S \\
L \\
s \\
5 \\
S \\
- \\
S \\
s \\
S \\
S \\
- \\
S\end{array}$ & $\begin{array}{l}\mathbf{s} \\
\mathbf{s} \\
\mathbf{S} \\
\mathbf{S} \\
- \\
\mathbf{S} \\
\mathbf{L} \\
\mathbf{S} \\
\mathbf{s} \\
\mathbf{S} \\
\mathbf{S} \\
\mathbf{S} \\
\mathbf{S} \\
- \\
\mathbf{s} \\
\mathbf{s} \\
\mathbf{S}\end{array}$ & $\begin{array}{l}\mathbf{S} \\
\mathbf{S} \\
\mathbf{S} \\
\mathbf{S} \\
\mathbf{S} \\
\mathbf{S} \\
\mathrm{L} \\
\mathbf{S} \\
\mathbf{S} \\
\mathbf{S} \\
\mathbf{S} \\
\mathbf{S} \\
\mathbf{S} \\
\mathbf{-} \\
\mathbf{S} \\
\mathbf{S} \\
\mathbf{S}\end{array}$ \\
\hline
\end{tabular}

\begin{tabular}{|c|c|}
\hline & sont \\
\hline $\begin{array}{l}3= \\
L\end{array}$ & 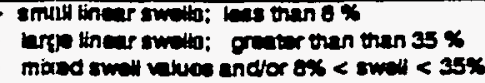 \\
\hline $\begin{array}{l}\text { AB } \\
\text { MO } \\
\text { PEEA } \\
\text { PEMA } \\
\text { PPCEM } \\
\text { PPGO } \\
\text { MPG }\end{array}$ & 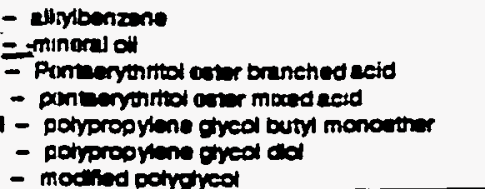 \\
\hline
\end{tabular}

$27-4$ 


\section{COMPATIBILITY OF REFRIGERANTS AND LUBRICANTS . WITH ENGINEERING PLASTICS}

\section{Objectives:}

- To provide compatibility information for engineering plastics with potential substitutes for CFC refrigerants and with suitable lubricants.

- To obtain data on changes in the mechanical properties of selected plastics after thermal aging in refrigerant-lubricant mixtures.

Results:

Imagination Resources, Inc., has completed this research under contract with ARTI. Detailed results are presented in the final report, DOE/CE/23810-15, Compatibility of Refrigerants and Lubricants with Engineering Plastics, December 1993, by Richard C. Cavestri, $\mathrm{PhD}$ (RDB \#4103, 182 pages).

This research project examined the compatibility of ten refrigerants and seven lubricants with twenty-three engineering plastics:

\begin{tabular}{|c|c|}
\hline Refrigerants & Lubricants \\
\hline HCFC-22 & naphthenic mineral oil (ISO 32) \\
\hline HCFC-123 & alkylbenzene (ISO 32) \\
\hline HCFC-124 & polypropylene glycol butyl monoether (ISO 32) \\
\hline HCFC-142b & polypropylene glycol diol (ISO 32) \\
\hline HFC-32 & modified polyglycol (ISO 32) \\
\hline HFC-125 & pentaerythritol ester, mixed-acid (ISO 32) \\
\hline HFC-134 & pentaerythritol ester, branched-acid (ISO 22) \\
\hline HFC-134a & \\
\hline HFC-143a & \\
\hline C-152 & \\
\hline
\end{tabular}




\section{Engineering Plastics Tested}

acetal

acrylonitrile-butadiene-styrene (ABS)

liquid crystal polymer (LCP)

modified polyetherimide

modified polyphenylene oxide

nylon $6 / 6$

phenolic

polyamide-imide (2 types)

polyaryletheretherketone

polyaryletherketone (PEK)

polyarylsulfone polybutylene terephthalate (PBT)

polycarbonate

polyetherimide

polyethylene terephthalate (PET)

polyimide thermoset (2 types)

polyphenylene sulfide (PPS)

polyphthalamide

polypropylene

(PEEK) polytetrafluoroethylene (PTFE)

polyvinylidene fluoride

Lubricant Immersion Studies: The plastic specimens were evaluated after 14-day exposures in pure lubricants at $60^{\circ} \mathrm{C}\left(140^{\circ} \mathrm{F}\right)$ and $100^{\circ} \mathrm{C}\left(212^{\circ} \mathrm{F}\right)$. Each plastic was affected to some extent by the lubricants. In general, weight and dimensional changes were in the plus or minus $1-2 \%$ range. However, the ABS specimens exhibited relatively larger changes in all the lubricants (in the 5-15\% range).

Refrigerant Immersion Studies: The plastics were evaluated at ambient room temperature and $60^{\circ} \mathrm{C}\left(140^{\circ} \mathrm{F}\right)$ in pure refrigerant for 14 days at the saturation pressure of the refrigerant. All refrigerants had some effect on the plastics; generally, weight increase and some softening of the plastics. HFC refrigerants seem to have the least effect on the plastics. The ABS plastic failed (e.g., dissolved or deformed) in HCFC-22, HFC-32, HCFC-123, HCFC-124, HFC-134, and HFC-152a. The polycarbonate and the modified polyphenylene oxide plastics failed in $\mathrm{HCFC}-123$.

Stress Crack-Creep Rupture Tests: Linear creep was measured for plastic test bars submerged in an ISO 32 cSt branched acid polyolester lubricant with $40 \%$ refrigerant concentrations (by weight) at $20^{\circ} \mathrm{C}\left(68^{\circ} \mathrm{F}\right)$ for 14 days. Each plastic was weight loaded at $25 \%$ of its ultimate tensile capability to stress the gage area of specimen test bars. The resultant deformation under load information provided the creep modulus arising from the exposure effects of synthetic lubricants with the differing refrigerants.

Plastic creep appeared to be nearly the same for all refrigerants. However, plastics exposed to HCFC-22 exhibited slightly lower creep rates than when exposed to the other nine refrigerants. Two plastics that routinely failed (e.g., broke within one hour) were ABS and modified polyphenylene oxide. HCFC-123, as expected, induced a pronounced increase in plastic creep, but did not promote rupture of the plastic test specimens. 
Refrigerant-Lubricant Thermal Aging Tests: Thermal aging tests on the twenty-three plastic specimens in seventeen refrigerant-lubricant combinations were completed. These tests were performed for 14 days at $150^{\circ} \mathrm{C}\left(300^{\circ} \mathrm{F}\right)$ and at refrigerant pressures from 1,900 to $2,070 \mathrm{kPa}$ (275 to $300 \mathrm{psia})$. Due to its higher reactivity, HCFC-123 aging tests were performed at $125^{\circ} \mathrm{C}\left(260^{\circ} \mathrm{F}\right)$ and at $105^{\circ} \mathrm{C}\left(220^{\circ} \mathrm{F}\right)$. Physical changes were observed, dimensional changes measured, and specimen tensile properties were compared to the original, unexposed specimens.

- After aging, the plastics exhibited minimal dimensional and weight changes (i.e., generally within plus or minus $2 \%$ ). However, the phenolic, polyvinylidene fluoride, and polypropylene plastic specimens exhibited the largest dimensional and weight changes (generally 5-20\%). As compared to the tensile tests performed on non-aged plastic test bars, the aged specimens exhibited large reductions in tensile capabilities (i.e., changes in tensile strengths ranged from a $30 \%$ gain to a $50 \%$ loss, changes in elongation ranged from a $10 \%$ increase to a $85 \%$ loss). Hence, as a result of environmental embrittlement, many plastics broke after a much smaller elongation under a much lower tensile load; as compared to the non-aged specimens. 


\section{ELECTROHYDRODYNAMIC (EHD) ENHANCEMENT \\ OF POOL AND IN-TUBE BOILING \\ OF ALTERNATIVE REFRIGERANTS}

\section{Objectives:}

- To construct a test rig that can measure improvements with in-tube boiling and in-tube .condensation heat transfer performance when utilizing EHD enhancement technology.

- To ascertain the heat transfer benefits on pool boiling with HCFC-123/lubricant on single and muitiple enhanced tubes when utilizing EHD techniques.

Results:

The University of Maryland completed this research under contract with ARTI. The final report detailing the pool boiling test results and the fabrication and qualification of the intube apparatus is available under DOE report number DOE/CE/23810-17, EHD Enhancement of Pool and In-Tube Boiling of Alternative Refrigerants, August 1993, by M. M. Ohadi, S. Dessiatoun, A. Singh, and M. A. Faani (RDB \#3A16, 62 pages).

This project accomplished three major tasks: (1) literature search on prior EHD research, (2) EHD pool boiling experiments with HCFC-123 and HFC-134a, and (3) design, fabrication, and shakedown of an EHD in-tube boiling/condensation test rig.

For pool boiling, higher applied electric potentials resulted in higher EHD-induced effects that promoted refrigerant bubble break-up and increased bubble departure speeds; collectively leading to higher heat transfer rates. For pool-boiling with HCFC-123 and HFC-134a, it was reported that the heat transfer rates increased $5-8$ fold, as compared to the non-EHD enhanced runs. This depended on whether or not $2 \%$ lubricant concentration was added and on whether mesh-type or straight-wire electrodes were utilized. 


\section{ACCELERATED SCREENING METHODS FOR PREDICTING LUBRICANT PERFORMANCE IN REFRIGERANT COMPRESSORS}

\section{Objective:}

To propose or devise a bench test device for conducting lubricity tests that simulates conditions in refrigeration and air-conditioning compressors.

\section{Results:}

The University of Illinois at Urbana-Champaign has completed this research under contract with ARTI. A detailed report of results is presented in the final, DOE report number DOE/CE/23810-45, Accelerated Screening Methods for Predicting Lubricant Performance in Refrigerant Compressors, November 1994, by C. Cusano, H. Yoon, and C. Poppe (RDB \#5109, 146 pages).

Refrigerants and lubricants tested in the program were:

$\begin{array}{lll}\text { CFC-12 and mineral oil } & - & \text { CFC baseline } \\ \text { HCFC-22 and mineral oil } & - & \text { HCFC baseline } \\ \text { HFC-134a and pentaerythritol ester lubricants } & -- & \text { HFC evaluation } \\ \text { R-32/125/134a }(30 / 10 / 60 \%) \text { and ester lubricants } & -- & \text { blend evaluation }\end{array}$

This investigation was performed in two parts. Part I of the study was a comparison between data obtained from a Falex ${ }^{\otimes}$ specimen tester versus data obtained from the University's of Illinois' proprietary high pressure tribometer (HPT). The main purpose of this comparison was to determine if the controlled environment and the lower loads used with the HPT produce different lubricant rankings than those obtained from the Falex ${ }^{\circledast}$ tests. Although the rankings from the HPT did not always correlate with those from the Falex ${ }^{\circledast}$ tester, the HPT resulted in consistent rankings at different loads and speeds. In Part II, the HPT is used to approximately simulate specific critical contacts in compressors to determine the extent to which the. HPT could predict lubricant performance. A comparison was made between data supplied from compressor manufacturers of compressor component tests and those obtained from the HPT. For comparison purposes, each lubricant was also tested and ranked based on results obtained in an air environment with the HPT and a Four-Ball test machine.

The goal of the research was to recommend a novel bench tester which could be developed to predict lubricant performance in refrigerant compressors. However, the data obtained did not provide a clear approach to accomplish this goal. 


\section{Part I: Comparison of HPT Results with Falex ${ }^{\otimes}$ Test Results}

Qualitative Falex ${ }^{\circledast}$ results (e.g., best, intermediate, worst) provided by three airconditioning and refrigeration compressor manufacturers were compared against data measured in the University of Illinois' proprietary high pressure tribometer (HPT). The contact geometries, speeds, and refrigerant-lubricant.mixtures used by the manufacturers in obtaining the Falex ${ }^{\circledast}$ results were modeled in the HPT. However, whereas the Falex ${ }^{\circledast}$ tests.were conducted at room temperature and atmospheric pressure (with refrigerant bubbled through the lubricant) at relatively high contact loads, the HPT tests were performed at temperatures; pressures and load conditions that better approximated critical contacts in scroll and reciprocating compressors. Lubricant rankings obtained from the Falex $^{\oplus}$ tests were compared to rankings of the same lubricants tested in the HPT. The following contact pairs were evaluated for friction and wear (e.g., wear scars, wear surface, and surface roughness) in unidirectional or oscillating contact tests:

- SAE 333 aluminum pin on gray cast iron disk (scroll compressor)

- hardened drill rod pin on SAE 356 aluminum disk (reciprocating compressor)

- carburized 1018 low carbon steel pin on SAE 380 die cast aluminum pad (reciprocating compressor)

- carburized 1018 low carbon steel pin on gray cast iron disk (reciprocating compressor)

The report draws the following conclusions on the Falex ${ }^{\otimes}$ and HPT comparisons:

1). Lubricant ranking correlation between the HPT and Falex ${ }^{(1)}$ tester is obtained only when relatively large wear differences existed between the lubricants.

2). For a given refrigerant, and based on statistical significance, lubricant ranking obtained by means of the HPT remained unchanged even if the loads and speeds were changed.

3). A lubricant-refrigerant mixture which produces relatively low wear will not necessarily produce relatively low friction.

4). The ranking of the lubricants can be a function of the material pairs in contact. A refrigerant-lubricant combination can have excellent wear characteristics with one contact pair and poor wear characteristics with another.

5). For the operating conditions examined, $R-134 a$ and the $R-32 / 125 / 134 a$ refrigerants with ester lubricants generally resulted in higher wear than the baseline R-12 and $\mathrm{R}-22$ refrigerants with mineral oil.

\section{Part II: Comparison of HPT Results with Compressor Component Testing}

Qualitative compressor component results (e.g., best, intermediate, worst) provided by four air-conditioning and refrigeration compressor manufacturers were compared against data measured in the HPT. The HPT operating conditions were chosen to approximately 
simulate those found at critical contacts in compressors. The HPT tests were conducted in both lubricant-refrigerant and lubricant-air environments. The lubricant-air tests helped establish the influence of the refrigerant on the behavior and ranking of the lubricants.

The following contact pairs were evaluated for friction and wear (e.g., wear scars, wear surface, and surface roughness) in the HPT and compared to compressor component tests:

Compressor application simulation

reciprocating compressor:

wrist pin/bearing contact

reciprocating compressor:

piston ring/cylinder ring contact

rotary compressor:

vane/piston contact contact pairs

$380^{\circ}$ die cast aluminum pad with carburized 1018 steel pin

ductile cast iron disk with carburized 1018 steel pin

sintered ferrous metal disk with sintered

ferrous metal pin

The report draws the following conclusions on the HPT and Four-Ball Tester versus compressor component testing:

1). None of the specimen testers produced data which exactly correlated with the compressor component testing.

2). For given conditions and material pairs, the presence of R-134a with any lubricant consistently increased wear on the specimens as compared to the same lubricant acting alone.

3). As in Part I, a lubricant-refrigerant mixture which produces relatively low wear will not necessarily produce low friction.

4). The HPT data obtained suggests that lubricant ranking is affected by environmental conditions (e.g., pressure and temperature). 